\title{
Anomaly corrected heterotic horizons
}

\section{A. Fontanella, ${ }^{a}$ J.B. Gutowski ${ }^{a}$ and G. Papadopoulos ${ }^{b}$}

\author{
${ }^{a}$ Department of Mathematics, University of Surrey, \\ Guildford, GU2 7XH, U.K. \\ ${ }^{b}$ Department of Mathematics, King's College London, \\ Strand, London WC2R 2LS, U.K. \\ E-mail: a.fontanella@surrey.ac.uk, j.gutowski@surrey.ac.uk, \\ george.papadopoulos@kcl.ac.uk
}

\begin{abstract}
We consider supersymmetric near-horizon geometries in heterotic supergravity up to two loop order in sigma model perturbation theory. We identify the conditions for the horizons to admit enhancement of supersymmetry. We show that solutions which undergo supersymmetry enhancement exhibit an $\mathfrak{s l}(2, \mathbb{R})$ symmetry, and we describe the geometry of their horizon sections. We also prove a modified Lichnerowicz type theorem, incorporating $\alpha^{\prime}$ corrections, which relates Killing spinors to zero modes of near-horizon Dirac operators. Furthermore, we demonstrate that there are no $\mathrm{AdS}_{2}$ solutions in heterotic supergravity up to second order in $\alpha^{\prime}$ for which the fields are smooth and the internal space is smooth and compact without boundary. We investigate a class of nearly supersymmetric horizons, for which the gravitino Killing spinor equation is satisfied on the spatial cross sections but not the dilatino one, and present a description of their geometry.
\end{abstract}

KeYwords: Black Holes in String Theory, Supergravity Models

ARXIV EPRINT: 1605.05635 


\section{Contents}

1 Introduction 1

2 Supersymmetric heterotic near-horizon geometries 4

2.1 Near horizon fields 4

2.2 Supersymmetry 5

2.2.1 The gravitino equation 5

2.2.2 Dilatino and gaugino KSEs 6

3 Solutions with $\phi_{+}^{[0]} \equiv 0 \quad 8$

4 Solutions with $\phi_{+}^{[0]} \not \equiv 0 \quad 10$

$\begin{array}{lll}4.1 & \text { Independent KSEs } & 12\end{array}$

5 Supersymmetry enhancement $\quad 12$

6 Geometry 14

6.1 Horizons with $G_{2}$ structure $\quad 14$

$\begin{array}{ll}6.2 \text { Horizons with SU(3) structure } & 15\end{array}$

$\begin{array}{lll}6.3 & \text { Horizons with } \mathrm{SU}(2) \text { structure and } 6 \text { supersymmetries } & 16\end{array}$

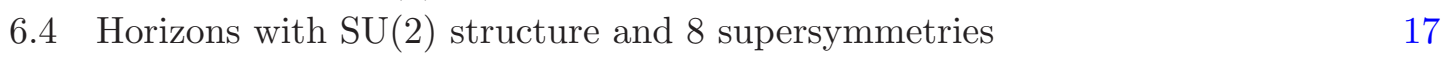

$\begin{array}{lll}7 & \text { Global properties } & 17\end{array}$

$\begin{array}{ll}7.1 & \text { Maximum principle on } h^{2} \\ 7.2 & 17\end{array}$

$\begin{array}{lll}7.2 & \text { Lichnerowicz type theorems } & 19\end{array}$

8 Nearly supersymmetric horizons $\quad 20$

8.1 Description of the backgrounds 20

$\begin{array}{ll}8.2 & \text { Additional parallel spinors } \\ 8.3 & 21\end{array}$

8.3 Nearly supersymmetric horizons with $G_{2}$ holonomy 21

$\begin{array}{lll}\text { 8.3.1 A symmetry of horizon section } & 21\end{array}$

$\begin{array}{lll}\text { 8.3.2 Geometry } & 24\end{array}$

9 Nearly supersymmetric horizons with additional parallel spinors $\quad \mathbf{2 5}$

9.1 Nearly supersymmetric horizons with SU(3) holonomy 25

9.1.1 Symmetries of horizon section 25

$\begin{array}{lll}9.1 .2 & \text { Geometry } & 29\end{array}$

9.2 Nearly supersymmetric horizons with SU(2) holonomy 31

$\begin{array}{lll}9.2 .1 & \text { Assumptions and definitions } & 31\end{array}$

9.2.2 Symmetries of the horizon section 31

$\begin{array}{lll}9.2 .3 \text { Geometry } & 32\end{array}$

10 Conclusions $\quad 34$ 
$\begin{array}{lll}\text { A.1 Spin connection and curvature } & 36\end{array}$

$\begin{array}{lll}\text { A.2 Bosonic field equations } & 37\end{array}$

B Further simplification of the KSEs $\quad 38$

B.1 Elimination of conditions (4.19), (4.21), (4.23), (4.25) 38

B.2 Elimination of $u$-dependent parts of (4.18) and (4.20) 40

$\begin{array}{ll}\text { C A consistency condition } & 41\end{array}$

D Lichnerowicz theorem computation $\quad 42$

$\begin{array}{lll}\text { D.1 Computations at zeroth order in } \alpha^{\prime} & 42\end{array}$

D.2 Computations at first order in $\alpha^{\prime} \quad 45$

$\begin{array}{lll}\text { E } & \mathrm{AdS}_{n+1} \text { as warped product over } \mathrm{AdS}_{n} & 47\end{array}$

\section{Introduction}

The effect of higher order corrections to supergravity solutions is of considerable interest, perhaps most notably for our understanding of quantum corrections to black holes. This is important in determining how string theory may resolve black hole singularities, as well as the investigation of the properties of black holes away from the limit $\alpha^{\prime} \rightarrow 0$. In higher dimensions the four dimensional uniqueness theorems [1-7] no longer hold, and there are exotic types of black hole solutions, such as the five dimensional black rings [8]. For ten and eleven dimensional supergravity, it is expected that there is a particularly rich structure of black objects, and the classification of these is ongoing. Progress has recently been made in the classification of the near-horizon geometries of supersymmetric black holes. Nearhorizon geometries of extremal black holes in supergravity are known to generically undergo supersymmetry enhancement. This has been proven by analysing the global properties of such solutions via generalized Lichnerowicz theorems [9-12], and making use of index theory arguments [13]. One consequence of the supersymmetry enhancement is that all such nearhorizon geometries exhibit an $\mathfrak{s l}(2, \mathbb{R})$ symmetry. However, it is not apparent that these properties persist after including string theory corrections.

There are several approaches to investigate how $\alpha^{\prime}$ corrections can change the event horizons of black holes. Many black holes have $\mathrm{AdS}_{p} \times S^{q}$ near-horizon geometries and as it is expected that the symmetries of such backgrounds persist in quantum theory, only the radii of the sphere and AdS receive $\alpha^{\prime}$ corrections. However, we expect that exotic black holes in higher dimensions need not necessarily have such near-horizon geometries.

Another approach, in the context of supersymmetric black holes in four and five dimensions, is to assume that the corrected near horizon geometries undergo an enhancement of supersymmetry in the near-horizon limit, which simplifies considerably the analysis of the Killing spinor equations. It is known that all supergravity $D=4$ and $D=5$ black 
holes undergo supersymmetry enhancement in the near-horizon limit [14-16]. In particular, the five dimensional BMPV black hole [17] undergoes supersymmetry enhancement from $N=4$ to $N=8$ (maximal supersymmetry) in the near-horizon limit [18]. Also, the supersymmetric asymptotically $\mathrm{AdS}_{5}$ black hole of [19] undergoes supersymmetry enhancement from $N=2$ to $N=4$ (half-maximal supersymmetry) in the near-horizon limit. However it is not clear in general why one expects that the $\alpha^{\prime}$ corrections should preserve this property.

The first systematic classification of supersymmetric near-horizon geometries in a higher derivative theory in five dimensions [20] was done in [21], in which the only assumption made was that the solutions should preserve the minimal amount of supersymmetry. The five dimensional theory reduces to ungauged five-dimensional supergravity coupled to arbitrarily many vector multiplets when the higher derivative corrections are set to zero. In this limit, it is known that near-horizon geometries are maximally supersymmetric with constant scalars [22], which is consistent with the standard picture of the attractor mechanism. In contrast, when higher derivative terms are turned on, the list of near-horizon geometries determined in [21] includes not only the maximally supersymmetric geometries (which were classified in [23]), but also a set of regular non-maximally supersymmetric solutions, on making use of a result of [24]. Although it is unclear if these particular near-horizon geometries can be extended to a full black hole solution, the existence of such a solution proves that for certain supergravity theories, the presence of higher derivative terms can change how supersymmetry is enhanced for near-horizon solutions.

In this paper, we consider how higher derivative corrections to ten dimensional supergravity affect the geometry and supersymmetry of near-horizon solutions. We shall choose to begin this work by investigating heterotic theory which includes $\alpha^{\prime}$ corrections up to two loops in sigma model perturbation theory. This choice is motivated by two factors. Firstly, from the perspective of the standard supergravity, much more is known about the geometric structure of generic supersymmetric solutions, and near-horizon geometries. In particular, as a consequence of the spinorial geometry classification techniques developed in $[25,26]$ which were then combined with a global analysis of near-horizon geometries in [27], there exists a full classification of all possible supersymmetric near-horizon geometries in the heterotic supergravity. Secondly, the structure of higher derivative correction terms in the field equations, and in the Killing spinor equations, is significantly simpler for the heterotic theory when compared to the types of terms which arise in type II supergravity [29-32], and associated references.

The method we shall use to prove our results is that first we solve the Killing spinor equations in the near-horizon lightcone directions, and then simplify the remaining conditions as much as possible using both the local field equations and Bianchi identities, as well as global analysis. For the global analysis, we shall assume that the spatial cross-section of the event horizon is smooth and compact, without boundary, and that all near-horizon fields are also smooth. As a result of this analysis, we find that there are no $\mathrm{AdS}_{2}$ solutions (at zero and first order in $\alpha^{\prime}$ ) to heterotic supergravity, which completes the classification of heterotic AdS solutions in [33]. We also show that all of the conditions of supersymmetry reduce to a pair of gravitino KSEs and a pair of algebraic KSEs on the spatial 
horizon sections. The latter are associated to the dilatino KSE. Throughout, we allow for all near-horizon data, including the spinors, to receive $\alpha^{\prime}$ corrections.

Using these conditions, we show that there is automatic supersymmetry enhancement at both zero and first order in $\alpha^{\prime}$ in the case for which there exists negative light-cone chirality Killing spinor $\eta_{-}$up to $\mathcal{O}\left(\alpha^{\prime 2}\right)$ which does not vanish at zeroth order in $\alpha^{\prime}$. In this case the supersymmetry enhancement is obtained via the same mechanism as for the near-horizon geometries considered in [27] without $\alpha^{\prime}$ corrections, and the solution admits an $\mathfrak{s l}(2, \mathbb{R})$ symmetry. Such horizons admit 2, 4, 6 and 8 Killing spinors and their geometry is similar to that of horizons with vanishing anomaly contribution examined in [27]. The remaining case, for which the negative light-cone chirality spinors vanish at zeroth order in $\alpha^{\prime}$ remains open. We have investigated global aspects of these solutions by considering $\alpha^{\prime}$ corrections to the global analysis carried out in [27], and also by constructing generalized Lichnerowicz theorems analogous to those proven in [9-12], again incorporating $\alpha^{\prime}$ corrections. However, in both cases, there is an undetermined sign in the $\mathcal{O}\left(\alpha^{\prime 2}\right)$ terms appearing, which precludes the extension of the maximum principle arguments to first order in $\alpha^{\prime}$.

We also consider a class of near-horizon solutions which are "nearly" supersymmetric. These are not supersymmetric but some of their KSEs are satisfied. This is motivated by the existence of WZW type of solutions to the heterotic theory with constant dilaton. It is known that such solutions solve the gravitino KSE but not the dilatino one. In the present case, we consider horizons for which one of the gravitino KSEs is satisfied ${ }^{1}$ on the spatial horizon section up order $\mathcal{O}\left(\alpha^{\prime 2}\right)$ but not the other and the algebraic KSEs. After some assumptions on the form of the fields, we give a complete description of the geometry of such solutions.

This paper is organized as follows. In section 2, we present the fields of heterotic near-horizon geometries and we integrate up the KSEs along the lightcone directions. In sections 3 and 4, we identify the independent KSEs by examining the various cases that can occur and in the process, prove that there are no $\mathrm{AdS}_{2}$ solutions. In section 5, we determine the conditions under which the horizons exhibit supersymmetry enhancement, and in section 6 we give the geometry of the horizon sections. In section 7 , we generalize the global analysis presented near-horizon geometries in [27] to include $\alpha^{\prime}$ corrections. However because of a $\mathcal{O}\left(\alpha^{\prime 2}\right)$ sign ambiguity, it is not possible to prove that the horizon section admits a $G_{2}$ structure compatible with a connection with skew-symmetric torsion, as is the case at zeroth order in $\alpha^{\prime}$. We also generalize the Lichnerowicz type theorems to higher orders in $\alpha^{\prime}$. Once again, $\mathcal{O}\left(\alpha^{\prime 2}\right)$ sign ambiguity means that it is not possible to prove that zero modes of the horizon Dirac equation (at zero and first order in $\alpha^{\prime}$ ) satisfy the Killing spinor equations to the same order in $\alpha^{\prime}$, although the algebraic Killing spinor involving the 2 -form gauge field is satisfied to the required order in $\alpha^{\prime}$. In sections 8 and 9 , we examine the geometry of nearly supersymmetric horizons focusing on those that admit a solution to the gravitino KSE on the horizon spatial section, and in section 10 we give our conclusions.

\footnotetext{
${ }^{1}$ Such solutions are not supersymmetric, and furthermore the spacetime gravitino KSE is not necessarily satisfied.
} 
The paper contains several appendices. In appendix A, we summarize some key formulae that are used throughout in the computations of the paper and present the field equations of the theory. In appendix B, we provide the details of part of the proof to identify the independent KSEs on the spatial horizon section. In section C, we present a formula which relates the gravitino KSE to the gaugino KSE which is instrumental in the investigation of the geometry of nearly supersymmetric horizons. In appendix D, we present further detail of the proof of the Lichnerowicz type theorem for the heterotic theory, and in appendix E, we describe how $\mathrm{AdS}_{n+1}$ can be written as a warped product over $\mathrm{AdS}_{n}$, and describe how such constructions are inconsistent with our assumptions on the global structure and regularity of the solutions.

\section{Supersymmetric heterotic near-horizon geometries}

\subsection{Near horizon fields}

The metric near a smooth killing horizon expressed in Gaussian null co-ordinates [34, 35] can be written as

$$
d s^{2}=2 \mathbf{e}^{+} \mathbf{e}^{-}+\delta_{i j} \mathbf{e}^{i} \mathbf{e}^{j}
$$

where we have used the frame

$$
\mathbf{e}^{+}=d u, \quad \mathbf{e}^{-}=d r+r h-\frac{1}{2} r^{2} \Delta d u, \quad \mathbf{e}^{i}=e^{i}{ }_{J} d y^{J},
$$

$i, j=1, \ldots, 8, u, r$ are the lightcone coordinates, and the 1 -form $h$, scalar $\Delta$ and $\mathbf{e}^{i}$ depend only on the coordinates $y^{I}, I=1, \ldots, 8$, transverse to the lightcone. The black hole stationary Killing vector field is identified with $\partial_{u}$. The induced metric on $\mathcal{S}$ is

$$
d s_{\mathcal{S}}^{2}=\delta_{i j} \mathbf{e}^{i} \mathbf{e}^{j}
$$

and $\mathcal{S}$ is taken to be compact, connected without boundary. We denote the Levi-Civita connection of $\mathcal{S}$ by $\tilde{\nabla}$, and the Levi-Civita connection of the $\mathrm{D}=10$ spacetime as $\nabla$.

For the other heterotic fields, we assume that the dilaton $\Phi$, and the real 3-form $H$, and non-abelian gauge potential $A$ admit well-defined near-horizon limits, and that $\partial_{u}$ is a symmetry of the full solution:

$$
\mathcal{L}_{\partial_{u}} \Phi=0, \quad \mathcal{L}_{\partial_{u}} H=0, \quad \mathcal{L}_{\partial_{u}} A=0 .
$$

In particular, this means that $\Phi=\Phi(y)$, and also

$$
H=\mathbf{e}^{+} \wedge \mathbf{e}^{-} \wedge N+r \mathbf{e}^{+} \wedge Y+W,
$$

where $N, Y$ and $W$ are $u, r$-independent 1,2 and 3-forms on $\mathcal{S}$ respectively, and we do not assume $d H=0$. Moreover,

$$
A=r \mathcal{P} \mathbf{e}^{+}+\mathcal{B},
$$

where $\mathcal{P}$ and $\mathcal{B}$ are a $r, u$-independent $G$-valued scalar and 1-form on $\mathcal{S}$ respectively. The non-abelian 2-form field strength $F$ is given by

$$
F=d A+A \wedge A \text {. }
$$


Our conventions for the heterotic theory including $\alpha^{\prime}$ corrections are consistent with those of [29]. We assume that the near-horizon data admit a Taylor series expansion in $\alpha^{\prime}$. We denote this expansion by

$$
\Delta=\Delta^{[0]}+\alpha^{\prime} \Delta^{[1]}+\mathcal{O}\left(\alpha^{2}\right)
$$

and similarly for all near-horizon data, including spinors. For the supersymmetric solutions, we shall assume that that there is at least one zeroth order in $\alpha^{\prime}$ Killing spinor, $\epsilon^{[0]} \neq 0$.

\subsection{Supersymmetry}

In the previous treatments of heterotic near-horizon geometries [27], it was assumed that the anomaly vanishes and so the Bianchi identity $d H=0$ was used to further simplify the structure of the 3 -form. Here, we shall not take $d H=0$ as there is a non-trivial contribution from the heterotic anomaly, and so the 3 -form takes the more general form given in (2.5).

We remark that the KSE of heterotic supergravity have been solved in [25] and [26], and so, the solutions to the KSEs which we consider here correspond to a subclass of the solutions in $[25,26]$. However for horizons the global assumptions on the spatial section $\mathcal{S}$, like compactness, allow the derivation of additional conditions on the spinors and on the geometry. So it is particularly useful to re-solve the KSEs, decomposing the spinors into positive and negative lightcone chiralities adapted for the Gaussian null basis (2.2), $\epsilon=\epsilon_{+}+\epsilon_{-}$, where

$$
\Gamma_{ \pm} \epsilon_{ \pm}=0, \quad \Gamma_{+-} \epsilon_{ \pm}= \pm \epsilon_{ \pm} .
$$

We shall then extract from the KSEs the conditions imposed on $\epsilon_{ \pm}$that will be useful to apply the global conditions on $\mathcal{S}$.

\subsubsection{The gravitino equation}

We begin by considering the gravitino equation

$$
\hat{\nabla}_{M} \epsilon \equiv \nabla_{M} \epsilon-\frac{1}{8} H_{M N_{1} N_{2}} \Gamma^{N_{1} N_{2}} \epsilon=\mathcal{O}\left(\alpha^{\prime 2}\right) .
$$

First, on examining the $M=-$ component of $(2.10)$ we find that

$$
\epsilon_{+}=\phi_{+}+\mathcal{O}\left(\alpha^{\prime 2}\right), \quad \epsilon_{-}=\phi_{-}+\frac{1}{4} r(h-N)_{i} \Gamma_{-} \Gamma^{i} \phi_{+}+\mathcal{O}\left(\alpha^{\prime 2}\right)
$$

where $\partial_{r} \phi_{ \pm}=0$. Next, on examining the $M=+$ component of (2.10), we find

$$
\phi_{-}=\eta_{-}+\mathcal{O}\left(\alpha^{\prime 2}\right), \quad \phi_{+}=\eta_{+}+\frac{1}{4} u(h+N)_{i} \Gamma_{+} \Gamma^{i} \eta_{-}+\mathcal{O}\left(\alpha^{\prime 2}\right),
$$

where $\partial_{r} \eta_{ \pm}=\partial_{u} \eta_{ \pm}=0$. In additon, the $M=+$ component of (2.10) implies a number of algebraic conditions:

$$
\left(\frac{1}{2} \Delta+\frac{1}{8}\left(h^{2}-N^{2}\right)-\frac{1}{8}(d h+Y+h \wedge N)_{i j} \Gamma^{i j}\right) \phi_{+}=\mathcal{O}\left(\alpha^{\prime 2}\right)
$$


and

$$
\left(-\frac{1}{2} \Delta-\frac{1}{8}\left(h^{2}-N^{2}\right)-\frac{1}{8}(d h+Y+h \wedge N)_{i j} \Gamma^{i j}\right) \eta_{-}=\mathcal{O}\left(\alpha^{\prime 2}\right),
$$

and

$$
\left(\frac{1}{4}\left(\Delta h_{i}-\partial_{i} \Delta\right) \Gamma^{i}-\frac{1}{32}(d h+Y)_{i j} \Gamma^{i j}(h-N)_{k} \Gamma^{k}\right) \phi_{+}=\mathcal{O}\left(\alpha^{\prime 2}\right) .
$$

We remark that (2.13) and (2.14) are equivalent to

$$
\begin{aligned}
\frac{1}{2} \Delta+\frac{1}{8}\left(h^{2}-N^{2}\right) & =\mathcal{O}\left(\alpha^{\prime 2}\right), \\
(d h+Y+h \wedge N)_{i j} \Gamma^{i j} \phi_{+} & =\mathcal{O}\left(\alpha^{\prime 2}\right),
\end{aligned}
$$

and

$$
(d h+Y+h \wedge N)_{i j} \Gamma^{i j} \eta_{-}=\mathcal{O}\left(\alpha^{\prime 2}\right),
$$

respectively. Furthermore, using these conditions, (2.15) can also be rewritten as

$$
\left(\frac{1}{4}\left(\Delta h_{j}-\partial_{j} \Delta\right)-\frac{1}{8}(h-N)^{k}(d h+Y+2 h \wedge N)_{j k}\right) \Gamma^{j} \phi_{+}=\mathcal{O}\left(\alpha^{\prime 2}\right) .
$$

Next, we consider the $M=i$ components of (2.10). This implies

$$
\tilde{\nabla}_{i} \phi_{+}+\left(\frac{1}{4}(N-h)_{i}-\frac{1}{8} W_{i j k} \Gamma^{j k}\right) \phi_{+}=\mathcal{O}\left(\alpha^{2}\right)
$$

and

$$
\tilde{\nabla}_{i} \eta_{-}+\left(\frac{1}{4}(h-N)_{i}-\frac{1}{8} W_{i j k} \Gamma^{j k}\right) \eta_{-}=\mathcal{O}\left(\alpha^{\prime 2}\right),
$$

together with the algebraic condition

$$
\begin{aligned}
\left(\tilde{\nabla}_{i}(h-N)_{j}+\frac{1}{2}\left(h_{i} N_{j}-h_{j} N_{i}\right)-\frac{1}{2}\left(h_{i} h_{j}-N_{i} N_{j}\right)\right. & \\
\left.-(d h-Y)_{i j}-\frac{1}{2} W_{i j k}(h-N)^{k}\right) \Gamma^{j} \phi_{+} & =\mathcal{O}\left(\alpha^{\prime 2}\right) .
\end{aligned}
$$

These conditions exhaust the content of (2.10).

\subsubsection{Dilatino and gaugino KSEs}

Next again ignoring $O\left(\alpha^{2}\right)$ terms we consider the dilatino KSE

$$
\left(\Gamma^{M} \nabla_{M} \Phi-\frac{1}{12} H_{N_{1} N_{2} N_{3}} \Gamma^{N_{1} N_{2} N_{3}}\right) \epsilon=\mathcal{O}\left(\alpha^{\prime 2}\right) .
$$

On making use of the previous conditions, it is straightforward to show that the dilatino KSE is equivalent to the following three conditions

$$
\left(\Gamma^{i} \tilde{\nabla}_{i} \Phi+\frac{1}{2} N_{i} \Gamma^{i}-\frac{1}{12} W_{i j k} \Gamma^{i j k}\right) \phi_{+}=\mathcal{O}\left(\alpha^{\prime 2}\right),
$$


and

$$
\left(\Gamma^{i} \tilde{\nabla}_{i} \Phi-\frac{1}{2} N_{i} \Gamma^{i}-\frac{1}{12} W_{i j k} \Gamma^{i j k}\right) \eta_{-}=\mathcal{O}\left(\alpha^{2}\right)
$$

and

$$
\left(\left(\Gamma^{i} \tilde{\nabla}_{i} \Phi-\frac{1}{2} N_{i} \Gamma^{i}-\frac{1}{12} W_{i j k} \Gamma^{i j k}\right)(h-N)_{\ell} \Gamma^{\ell}+Y_{i j} \Gamma^{i j}\right) \phi_{+}=\mathcal{O}\left(\alpha^{\prime 2}\right) .
$$

It remains to consider the gaugino KSE

$$
F_{M N} \Gamma^{M N} \epsilon=\mathcal{O}\left(\alpha^{\prime}\right)
$$

This implies the following conditions

$$
\left(2 \mathcal{P}+\tilde{F}_{i j} \Gamma^{i j}\right) \phi_{+}=\mathcal{O}\left(\alpha^{\prime}\right)
$$

and

$$
\left(-2 \mathcal{P}+\tilde{F}_{i j} \Gamma^{i j}\right) \eta_{-}=\mathcal{O}\left(\alpha^{\prime}\right)
$$

and

$$
\left(\frac{1}{4}\left(-2 \mathcal{P}+\tilde{F}_{i j} \Gamma^{i j}\right)(h-N)_{\ell} \Gamma^{\ell}+2(h \mathcal{P}+\mathcal{P B}-\mathcal{B P}-d \mathcal{P})_{i} \Gamma^{i}\right) \phi_{+}=\mathcal{O}\left(\alpha^{\prime}\right)
$$

where

$$
\tilde{F}=d \mathcal{B}+\mathcal{B} \wedge \mathcal{B}
$$

The conditions (2.28) and (2.29) imply that

$$
\mathcal{P}=\mathcal{O}\left(\alpha^{\prime}\right)
$$

and so $F=\tilde{F}+\mathcal{O}\left(\alpha^{\prime}\right)$. Therefore (2.27) is equivalent to

$$
\tilde{F}_{i j} \Gamma^{i j} \phi_{+}=\mathcal{O}\left(\alpha^{\prime}\right),
$$

and

$$
\tilde{F}_{i j} \Gamma^{i j} \eta_{-}=\mathcal{O}\left(\alpha^{\prime}\right),
$$

and

$$
\tilde{F}_{i j} \Gamma^{i j}(h-N)_{\ell} \Gamma^{\ell} \phi_{+}=\mathcal{O}\left(\alpha^{\prime}\right) .
$$

In order to simplify these conditions further, we shall first consider the two cases for which either $\phi_{+}^{[0]} \equiv 0$ or $\phi_{+}^{[0]} \not \equiv 0$. 


\section{Solutions with $\phi_{+}^{[0]} \equiv 0$}

Suppose that there exists a Killing spinor $\epsilon$ with $\epsilon^{[0]} \not \equiv 0$, but $\phi_{+}^{[0]} \equiv 0$. Such a spinor must therefore have $\eta_{-}^{[0]} \not \equiv 0$, and hence it follows that

$$
h^{[0]}+N^{[0]}=0 .
$$

Then (2.21) implies that

$$
d\left\|\eta_{-}^{[0]}\right\|^{2}=-\left\|\eta_{-}^{[0]}\right\|^{2} h^{[0]} .
$$

In particular, this condition implies that if $\eta_{-}^{[0]}$ vanishes at any point on the horizon section, then $\eta_{-}^{[0]}=0$ everywhere. So, $\eta_{-}^{[0]}$ must be everywhere non-vanishing.

On taking the divergence of (3.2), and making use of the $N_{1}=+, N_{2}=-$ component of the 2-form gauge potential field equation (A.13), one obtains the following condition

$$
\tilde{\nabla}^{[0] i} \tilde{\nabla}_{i}^{[0]}\left\|\eta_{-}^{[0]}\right\|^{2}-\left(2 \tilde{\nabla}^{i} \Phi^{[0]}+\left\|\eta_{-}^{[0]}\right\|^{-2} \tilde{\nabla}^{[0] i}\left\|\eta_{-}^{[0]}\right\|^{2}\right) \tilde{\nabla}_{i}^{[0]}\left\|\eta_{-}^{[0]}\right\|^{2}=0
$$

As $\left\|\eta_{-}^{[0]}\right\|^{2}$ is nowhere vanishing, an application of the maximum principle implies that $\left\|\eta_{-}^{[0]}\right\|^{2}=$ const., and hence (3.2) gives that

$$
h^{[0]}=0, \quad N^{[0]}=0 .
$$

These conditions, together with (2.16), imply that

$$
\Delta=\mathcal{O}\left(\alpha^{\prime 2}\right) .
$$

Then the dilaton field equation (A.15) implies that

$$
\tilde{\nabla}^{i} \tilde{\nabla}_{i}\left(e^{-2 \Phi}\right)=\frac{1}{6} e^{-2 \Phi} W_{i j k} W^{i j k}+\mathcal{O}\left(\alpha^{\prime}\right),
$$

and hence it follows that

$$
\Phi^{[0]}=\text { const }, \quad W^{[0]}=0 .
$$

Furthermore, this then implies that

$$
H=d u \wedge d r \wedge N+r d u \wedge Y+W+\mathcal{O}\left(\alpha^{\prime 2}\right),
$$

and hence

$$
d H=d u \wedge d r \wedge(d N-Y)-r d u \wedge d Y+d W+\mathcal{O}\left(\alpha^{\prime 2}\right) .
$$

As the ruij component on the r.h.s. of the Bianchi identity is $\mathcal{O}\left(\alpha^{\prime 2}\right)$ this implies that

$$
Y=d N+\mathcal{O}\left(\alpha^{\prime 2}\right)
$$

and in particular, $Y^{[0]}=0$.

Next consider the gauge equations. The +- component of the 2 -form gauge potential field equations (A.13) is

$$
\tilde{\nabla}^{i} N_{i}=\mathcal{O}\left(\alpha^{\prime 2}\right)
$$


Also, the $u$-dependent part of (4.18) implies that

$$
\tilde{\nabla}_{i}(h+N){ }_{j} \Gamma^{j} \eta_{-}=\mathcal{O}\left(\alpha^{\prime 2}\right),
$$

which gives that

$$
\tilde{\nabla}_{i}(h+N)_{j}=\mathcal{O}\left(\alpha^{\prime 2}\right) .
$$

Taking the trace of this expression, and using (3.14) yields

$$
\tilde{\nabla}^{i} h_{i}=\mathcal{O}\left(\alpha^{\prime 2}\right) .
$$

Next, recall that the gravitino KSE (4.22) implies

$$
\tilde{\nabla}_{i}\left\|\eta_{-}\right\|^{2}=-\frac{1}{2}(h-N)_{i}\left\|\eta_{-}\right\|^{2}+\mathcal{O}\left(\alpha^{2}\right) .
$$

Taking the divergence yields, together with (3.11) and (3.14) the condition

$$
\tilde{\nabla}^{i} \tilde{\nabla}_{i}\left\|\eta_{-}\right\|^{2}=\mathcal{O}\left(\alpha^{\prime 2}\right)
$$

which implies that $\left\|\eta_{-}\right\|^{2}=$ const $+\mathcal{O}\left(\alpha^{\prime 2}\right)$. Substituting back into (3.15) gives the condition $N=h+\mathcal{O}\left(\alpha^{\prime 2}\right)$, and hence (3.13) implies that

$$
\tilde{\nabla}_{i} h_{j}=\mathcal{O}\left(\alpha^{\prime 2}\right) .
$$

So, to summarize, for this class of solutions, we have obtained the following conditions on the fields

$$
\begin{aligned}
& N=h+\mathcal{O}\left(\alpha^{\prime 2}\right), \quad h^{[0]}=0, \quad Y=\mathcal{O}\left(\alpha^{\prime 2}\right), \quad \tilde{\nabla}_{i} h_{j}=\mathcal{O}\left(\alpha^{\prime 2}\right), \\
& \Delta=\mathcal{O}\left(\alpha^{2}\right), \quad H^{[0]}=0, \quad \Phi^{[0]}=\text { const },
\end{aligned}
$$

and it is straightforward to check that the generic conditions on $\phi_{+}$then simplify to

$$
\tilde{\nabla}_{i} \phi_{+}-\frac{1}{8} W_{i j k} \Gamma^{j k} \phi_{+}=\mathcal{O}\left(\alpha^{2}\right),
$$

and

$$
\left(\Gamma^{i} \tilde{\nabla}_{i} \Phi+\frac{1}{2} h_{i} \Gamma^{i}-\frac{1}{12} W_{i j k} \Gamma^{i j k}\right) \phi_{+}=\mathcal{O}\left(\alpha^{\prime 2}\right),
$$

and

$$
\tilde{F}_{i j} \Gamma^{i j} \phi_{+}=\mathcal{O}\left(\alpha^{\prime}\right) .
$$

The generic conditions on $\eta_{-}$also simplify to

$$
\tilde{\nabla}_{i} \eta_{-}-\frac{1}{8} W_{i j k} \Gamma^{j k} \eta_{-}=\mathcal{O}\left(\alpha^{2}\right)
$$

and

$$
\left(\Gamma^{i} \tilde{\nabla}_{i} \Phi-\frac{1}{2} h_{i} \Gamma^{i}-\frac{1}{12} W_{i j k} \Gamma^{i j k}\right) \eta_{-}=\mathcal{O}\left(\alpha^{\prime 2}\right)
$$

and

$$
\tilde{F}_{i j} \Gamma^{i j} \eta_{-}=\mathcal{O}\left(\alpha^{\prime}\right) .
$$

In the next section, we shall consider the case for which there exists a Killing spinor with $\phi_{+}^{[0]} \not \equiv 0$. It will be shown that the conditions (3.18) on the bosonic fields and the simplified KSEs listed above correspond to special cases of the corresponding conditions on the fields and simplified KSEs of $\phi_{+}^{[0]} \not \equiv 0$. In particular, this will allow the KSEs for $\phi_{+}^{[0]} \equiv 0$ and $\phi_{+}^{[0]} \not \equiv 0$ to be written in a unified way. 


\section{Solutions with $\phi_{+}^{[0]} \not \equiv 0$}

Suppose that there exists a Killing spinor $\epsilon$, with $\epsilon^{[0]} \not \equiv 0$ and $\phi_{+}^{[0]} \not \equiv 0$. Then consider (2.20); this implies that

$$
\tilde{\nabla}_{i}\left\|\phi_{+}\right\|^{2}=\frac{1}{2}\left(h_{i}-N_{i}\right)\left\|\phi_{+}\right\|^{2}+\mathcal{O}\left(\alpha^{\prime 2}\right)
$$

and (2.22) gives that

$$
\begin{aligned}
& \tilde{\nabla}_{i}(h-N)_{j}+\frac{1}{2}\left(h_{i} N_{j}-h_{j} N_{i}\right)-\frac{1}{2}\left(h_{i} h_{j}-N_{i} N_{j}\right) \\
&-(d h-Y)_{i j}-\frac{1}{2} W_{i j k}(h-N)^{k}=\mathcal{O}\left(\alpha^{\prime 2}\right) .
\end{aligned}
$$

Taking the divergence of (4.1), and using (2.20) together with the trace of (4.2), we find that

$$
\tilde{\nabla}^{i} \tilde{\nabla}_{i}\left\|\phi_{+}\right\|^{2}-h^{i} \tilde{\nabla}_{i}\left\|\phi_{+}\right\|^{2}=\mathcal{O}\left(\alpha^{2}\right) .
$$

An application of the maximum principle (see e.g. [36]) then yields the condition

$$
\tilde{\nabla}_{i}\left\|\phi_{+}\right\|^{2}=\mathcal{O}\left(\alpha^{\prime 2}\right) .
$$

To see this, note that to zeroth order in $\alpha^{\prime},(4.3)$ implies that $\tilde{\nabla}_{i}^{[0]}\left\|\phi_{+}^{[0]}\right\|^{2}=0$, on applying the maximum principle. Then (4.1) and (4.2) imply that $N^{[0]}=h^{[0]}$ and $Y^{[0]}=d h^{[0]}$; and from (2.16) we also have $\Delta^{[0]}=0$. Then it is useful to consider the field equations of the 2-form gauge potential (A.13), which imply that

$$
\tilde{\nabla}^{i}\left(e^{-2 \Phi} h_{i}\right)=\mathcal{O}\left(\alpha^{\prime}\right)
$$

and

$$
e^{2 \Phi} \tilde{\nabla}^{j}\left(e^{-2 \Phi} d h_{j i}\right)+\frac{1}{2} W_{i j k} d h^{j k}+h^{j} d h_{j i}=\mathcal{O}\left(\alpha^{\prime}\right),
$$

and the Einstein equations imply that

$$
\tilde{R}_{i j}+\tilde{\nabla}_{(i} h_{j)}-\frac{1}{4} W_{i m n} W_{j}^{m n}+2 \tilde{\nabla}_{i} \tilde{\nabla}_{j} \Phi=\mathcal{O}\left(\alpha^{\prime}\right) .
$$

Using (4.5), (4.6) and (4.7) it follows that ${ }^{2}$

$$
\begin{aligned}
\tilde{\nabla}^{i} \tilde{\nabla}_{i} h^{2}+(h-2 d \Phi)^{j} \tilde{\nabla}_{j} h^{2}= & \left.2 \tilde{\nabla}^{(i} h^{j)} \tilde{\nabla}_{(i} h_{j}\right) \\
& +\frac{1}{2}\left(d h-i_{h} W\right)_{i j}\left(d h-i_{h} W\right)^{i j}+\mathcal{O}\left(\alpha^{\prime}\right) .
\end{aligned}
$$

In particular, (4.8) implies that $\tilde{\nabla}^{[0] i} h_{i}^{[0]}=0$ on applying the maximum principle. It follows from (4.3) that

$$
\tilde{\nabla}^{[0] i} \tilde{\nabla}_{i}^{[0]}\left\langle\phi_{+}^{[0]}, \phi_{+}^{[1]}\right\rangle-h^{[0] i} \tilde{\nabla}_{i}^{[0]}\left\langle\phi_{+}^{[0]}, \phi_{+}^{[1]}\right\rangle=0 .
$$

\footnotetext{
${ }^{2}$ We remark that the condition (4.8) was also obtained in [27]. In that case, a bilinear matching condition was imposed in order to find $N^{[0]}=h^{[0]}, Y^{[0]}=d h^{[0]}$. Here we do not assume such a bilinear matching condition, but nevertheless we find the same condition.
} 
On multiplying this condition by $\left\langle\phi_{+}^{[0]}, \phi_{+}^{[1]}\right\rangle$ and integrating by parts, using $\tilde{\nabla}^{[0] i} h_{i}^{[0]}=0$, one finds that $\tilde{\nabla}_{i}^{[0]}\left\langle\phi_{+}^{[0]}, \phi_{+}^{[1]}\right\rangle=0$ as well. So, it follows that $\tilde{\nabla}_{i}\left\|\phi_{+}\right\|^{2}=\mathcal{O}\left(\alpha^{2}\right)$.

Then, (4.1) also implies that $N=h+\mathcal{O}\left(\alpha^{\prime 2}\right)$. Substituting these conditions back into (2.16), we find that $\Delta^{[1]}=0$ as well, so $\Delta=\mathcal{O}\left(\alpha^{\prime 2}\right)$. Also, (4.2) implies that

$$
Y-d h=\mathcal{O}\left(\alpha^{\prime 2}\right) .
$$

To summarize the conditions on the bosonic fields; we have shown that for solutions with $\phi_{+}^{[0]} \neq 0$, we must have

$$
\Delta=\mathcal{O}\left(\alpha^{2}\right), \quad N=h+\mathcal{O}\left(\alpha^{\prime 2}\right), \quad Y=d h+\mathcal{O}\left(\alpha^{\prime 2}\right)
$$

which implies that

$$
H=d\left(\mathbf{e}^{-} \wedge \mathbf{e}^{+}\right)+W+\mathcal{O}\left(\alpha^{\prime 2}\right) .
$$

The field equation (A.13) of the 2-form gauge potential can then be rewritten in terms of the near-horizon data as

$$
\begin{aligned}
\tilde{\nabla}^{i}\left(e^{-2 \Phi} h_{i}\right) & =\mathcal{O}\left(\alpha^{2}\right), \\
e^{2 \Phi} \tilde{\nabla}^{j}\left(e^{-2 \Phi} d h_{j i}\right)+\frac{1}{2} W_{i j k} d h^{j k}+h^{j} d h_{j i} & =\mathcal{O}\left(\alpha^{\prime 2}\right),
\end{aligned}
$$

and

$$
e^{2 \Phi} \tilde{\nabla}^{k}\left(e^{-2 \Phi} W_{k i j}\right)+d h_{i j}-h^{k} W_{k i j}=\mathcal{O}\left(\alpha^{\prime 2}\right) .
$$

In addition, $\mathcal{P}=\mathcal{O}\left(\alpha^{\prime}\right)$ and so $F=\tilde{F}+\mathcal{O}\left(\alpha^{\prime}\right)$. The $i, j$ component of the Einstein equation then simplifies to

$$
\begin{aligned}
\tilde{R}_{i j} & +\tilde{\nabla}_{(i} h_{j)}-\frac{1}{4} W_{i m n} W_{j}^{m n}+2 \tilde{\nabla}_{i} \tilde{\nabla}_{j} \Phi \\
& +\frac{\alpha^{\prime}}{4}\left(-2 d h_{i \ell} d h_{j}^{\ell}+\check{\tilde{R}}_{i \ell_{1}, \ell_{2} \ell_{3}} \check{\tilde{R}}_{j}^{\ell_{1}, \ell_{2} \ell_{3}}-\tilde{F}_{i \ell}{ }^{a b} \tilde{F}_{j}^{\ell}{ }_{a b}\right)=\mathcal{O}\left(\alpha^{2}\right) .
\end{aligned}
$$

Furthermore, dilaton field equation can be written as

$$
\begin{aligned}
\tilde{\nabla}^{i} & \tilde{\nabla}_{i} \Phi-h^{i} \tilde{\nabla}_{i} \Phi-2 \tilde{\nabla}^{i} \Phi \tilde{\nabla}_{i} \Phi-\frac{1}{2} h_{i} h^{i}+\frac{1}{12} W_{i j k} W^{i j k} \\
& +\frac{\alpha^{\prime}}{16}\left(2 d h_{i j} d h^{i j}+\tilde{F}_{i j}{ }^{a b} \tilde{F}^{i j}{ }_{a b}-\check{\tilde{R}}_{\ell_{1} \ell_{2}, \ell_{3} \ell_{4}} \check{\tilde{R}}^{\ell_{1} \ell_{2}, \ell_{3} \ell_{4}}\right)=\mathcal{O}\left(\alpha^{\prime 2}\right) .
\end{aligned}
$$

On making use of the conditions (4.11) on the bosonic fields, the KSEs on $\phi_{+}$then simplify further to

$$
\begin{aligned}
\tilde{\nabla}_{i} \phi_{+}-\frac{1}{8} W_{i j k} \Gamma^{j k} \phi_{+} & =\mathcal{O}\left(\alpha^{\prime 2}\right), \\
d h_{i j} \Gamma^{i j} \phi_{+} & =\mathcal{O}\left(\alpha^{\prime 2}\right), \\
\left(\Gamma^{i} \tilde{\nabla}_{i} \Phi+\frac{1}{2} h_{i} \Gamma^{i}-\frac{1}{12} W_{i j k} \Gamma^{i j k}\right) \phi_{+} & =\mathcal{O}\left(\alpha^{\prime 2}\right),
\end{aligned}
$$

and

$$
\tilde{F}_{i j} \Gamma^{i j} \phi_{+}=\mathcal{O}\left(\alpha^{\prime}\right)
$$


Furthermore, KSEs on $\eta_{-}$also simplify to

$$
\begin{aligned}
\tilde{\nabla}_{i} \eta_{-}-\frac{1}{8} W_{i j k} \Gamma^{j k} \eta_{-} & =\mathcal{O}\left(\alpha^{\prime 2}\right), \\
d h_{i j} \Gamma^{i j} \eta_{-} & =\mathcal{O}\left(\alpha^{\prime 2}\right), \\
\left(\Gamma^{i} \tilde{\nabla}_{i} \Phi-\frac{1}{2} h_{i} \Gamma^{i}-\frac{1}{12} W_{i j k} \Gamma^{i j k}\right) \eta_{-} & =\mathcal{O}\left(\alpha^{\prime 2}\right),
\end{aligned}
$$

and

$$
\tilde{F}_{i j} \Gamma^{i j} \eta_{-}=\mathcal{O}\left(\alpha^{\prime}\right) .
$$

In both cases above, (4.18) and (4.22) are a consequence of the gravitino KSE, (4.20) and (4.24) are associated to the dilatino KSE, while (4.21) and (4.25) are derived from the gaugino KSE. The two additional conditions (4.19) and (4.23) can be thought of as integrability conditions.

\subsection{Independent KSEs}

The KSEs we have stated in the previous sections (3.19)-(3.24) and (4.18)-(4.25) are not all independent. It turns out that the independent KSEs are

$$
\hat{\tilde{\nabla}} \eta_{ \pm} \equiv \tilde{\nabla}_{i} \eta_{ \pm}-\frac{1}{8} W_{i j k} \Gamma^{j k} \eta_{ \pm}=\mathcal{O}\left(\alpha^{\prime 2}\right)
$$

and

$$
\left(\Gamma^{i} \tilde{\nabla}_{i} \Phi \pm \frac{1}{2} h_{i} \Gamma^{i}-\frac{1}{12} W_{i j k} \Gamma^{i j k}\right) \eta_{ \pm}=\mathcal{O}\left(\alpha^{\prime 2}\right)
$$

This is the case irrespectively on whether $\phi_{+}^{[0]} \equiv 0$ or $\phi_{+}^{[0]} \neq 0$ though the conditions on the bosonic fields are somewhat different. The proof of this independence of the KSEs requires the use of field equations and Bianchi identities and it is rather involved. The details can be found in appendix B.

\section{$5 \quad$ Supersymmetry enhancement}

A key ingredient in the investigation of heterotic horizons is that supersymmetry always enhances. As a result horizons preserve 2, 4,6 and 8 supersymmetries [27]. However this is based on a global argument which we shall see does not necessarily apply to $\mathcal{O}\left(\alpha^{\prime 2}\right)$.

As a consequence we shall seek some alternative conditions to guarantee that supersymmetry enhances. In particular we shall show that if there exists a Killing spinor $\epsilon=\epsilon\left(\eta_{+}, \eta_{-}\right)$up to $\mathcal{O}\left(\alpha^{2}\right)$, ie $\eta_{-}$solves (4.26) and (4.27) up to $\mathcal{O}\left(\alpha^{2}\right)$, such that $\eta_{-}^{[0]} \neq 0$, and the horizon has $h^{[0]} \neq 0$, then there is automatic supersymmetry enhancement.

To prove this, it suffices to demonstrate that $h$ leaves all fields invariant and that it is covariantly constant with respect to the connection with torsion $\hat{\tilde{\nabla}}$ on $\mathcal{S}$. Indeed, first note that (B.15) implies that

$$
\hat{\tilde{\nabla}}_{i} h_{j} \equiv \tilde{\nabla}_{i} h_{j}-\frac{1}{2} W_{i j k} h^{k}=\mathcal{O}\left(\alpha^{\prime 2}\right) .
$$


In particular, to both zeroth and first order in $\alpha^{\prime}, h$ defines an isometry on $\mathcal{S}$, with $h^{2}=$ const $+\mathcal{O}\left(\alpha^{\prime 2}\right)$. Then the gauge equation (4.13) implies

$$
\mathcal{L}_{h} \Phi=\mathcal{O}\left(\alpha^{\prime 2}\right) .
$$

Also, the $u$-dependent part of (4.21) implies

$$
\left(i_{h} \tilde{F}\right)_{i} \Gamma^{i} \eta_{-}=\mathcal{O}\left(\alpha^{\prime}\right)
$$

which implies that $i_{h} \tilde{F}=\mathcal{O}\left(\alpha^{\prime}\right)$. So in the gauge for which $i_{h} \mathcal{B}=0$, one has

$$
\mathcal{L}_{h} \tilde{F}=\mathcal{O}\left(\alpha^{\prime}\right) .
$$

Next we consider $\mathcal{L}_{h} W$, where

$$
\mathcal{L}_{h} W=-\frac{\alpha^{\prime}}{2}\left(\operatorname{tr}\left(\left(i_{h} \check{R}\right) \wedge \check{R}\right)\right)+\mathcal{O}\left(\alpha^{\prime 2}\right),
$$

because $d h=i_{h} W+\mathcal{O}\left(\alpha^{\prime 2}\right)$. To evaluate this expression, note first that the integrability conditions of

$$
\hat{\tilde{\nabla}}_{i} \eta_{-}=\mathcal{O}\left(\alpha^{\prime 2}\right), \quad \hat{\tilde{\nabla}}_{i}\left(h_{\ell} \Gamma^{\ell} \eta_{-}\right)=\mathcal{O}\left(\alpha^{\prime 2}\right)
$$

are

$$
\hat{\tilde{R}}_{i j p q} \Gamma^{p q} \eta_{-}=\mathcal{O}\left(\alpha^{\prime 2}\right), \quad \hat{\tilde{R}}_{i j p q} \Gamma^{p q}\left(h_{\ell} \Gamma^{\ell} \eta_{-}\right)=\mathcal{O}\left(\alpha^{\prime 2}\right)
$$

from which we obtain the condition

$$
h^{\ell} \hat{\tilde{R}}_{i j \ell q}=\mathcal{O}\left(\alpha^{\prime 2}\right)
$$

and hence, as a consequence of (A.4),

$$
h^{\ell} \check{\tilde{R}}_{\ell q i j}=\mathcal{O}\left(\alpha^{\prime}\right) .
$$

Moreover,

$$
h^{\ell} \tilde{\tilde{R}}_{\ell q+-}=h^{i}(d h)_{i q}=\mathcal{O}\left(\alpha^{\prime 2}\right) .
$$

It follows that the contribution of $i_{h} \check{R}$ to the r.h.s. of (5.5) is of at least $\mathcal{O}\left(\alpha^{\prime}\right)$, and hence

$$
\mathcal{L}_{h} W=\mathcal{O}\left(\alpha^{\prime 2}\right) .
$$

So, we have shown that to both zero and first order in $\alpha^{\prime}$, the Lie derivative of the metric on $\mathcal{S}$, as well as $h, \Phi$ and $W$ with respect to $h$ vanishes, and the Lie derivative of $\tilde{F}$ with respect to $h$ vanishes to zeroth order in $\alpha^{\prime}$.

Supersymmetry is therefore enhanced, because if $\eta_{+}$satisfies (4.26) and (4.27), then so does $\eta_{-}^{\prime}=\Gamma_{-} h_{i} \Gamma^{i} \eta_{+}$. Conversely, if $\eta_{-}$satisfies (4.26) and (4.27), then so does $\eta_{+}^{\prime}=\Gamma_{+} h_{i} \Gamma^{i} \eta_{-}$. The proof of this makes use of the conditions (5.1), together with (5.2) and (4.19) and (4.23), and the reasoning is identical to that used in [27]. This establishes a 1-1 correspondence between spinors $\eta_{+}$and $\eta_{-}$satisfying (4.26) and (4.27), so the number of supersymmetries preserved is always even. 
Next we wish to determine whether a similar supersymmetry enhancement argument holds for $\eta_{+}$spinors. In particular if there exists a solution to (4.26) and (4.27) with $\eta_{+}^{[0]} \neq 0$ and $h^{[0]} \neq 0$, does this imply that the number of $\eta_{+}$solutions is equal to the number of $\eta_{-}$ solutions? This does not follow from a local analysis of (4.26) and (4.27), because there is no analogue of the condition (B.15) acting on $\eta_{+}$. Nevertheless, in [27] a global analysis was used in order to establish such a correspondence, by computing the Laplacian of $h^{2}$ and applying a maximum principle argument, in order to obtain (5.1) to zeroth order in $\alpha^{\prime}$. We shall revisit this analysis in section 7.1 including the $\alpha^{\prime}$ corrections.

\section{Geometry}

It is a consequence of the results of [27], see also section 7.1, that horizons with non-trivial fluxes preserve an even number of supersymmetries up to $\mathcal{O}\left(\alpha^{\prime}\right)$. Furthermore we have also demonstrated that such horizons with $\eta_{-}$Killing spinors preserve an even number of supersymmetries up to $\mathcal{O}\left(\alpha^{\prime 2}\right)$. It is straightforward to see that horizons with more than 8 supersymmetries are trivial, ie the rotation $h$ vanishes. Therefore, the heterotic horizons of interest preserve 2, 4, 6 and 8 supersymmetries.

Up to $\mathcal{O}\left(\alpha^{\prime}\right)$, the investigation of the geometry of all such horizons is identical to that given in [27] for heterotic horizons with closed 3-form field strength. Here we shall describe the geometry of the horizons that admit a $\eta_{-}$Killing spinor up to $\mathcal{O}\left(\alpha^{2}\right)$. We have seen that for such horizons $h$ is parallel with respect to the connection with torsion up to $\mathcal{O}\left(\alpha^{\prime 2}\right)$. Because of this, the geometry of such horizons is very similar to that of horizons with closed 3-form flux. The only differences between the geometries of the two cases are solely located in the modified Bianchi identity for the 3-form flux. As the two cases are similar, the description of the geometry will be brief.

\subsection{Horizons with $G_{2}$ structure}

Such horizons admit two supersymmetries up to $\mathcal{O}\left(\alpha^{\prime 2}\right)$. In particular $h$ satisfies (5.1). The spacetime locally can be described as a (principal) $\mathrm{SL}(2, \mathbb{R})$ fibration over a 7 -dimensional manifold $B^{7}$ which admits a metric $d \tilde{s}_{(7)}^{2}$ and a 3 -form $\tilde{H}_{(7)}$ such that the connection $\hat{\tilde{\nabla}}^{(7)}$ with torsion $\tilde{H}_{(7)}$ has holonomy contained in $G_{2}$. The spacetime metric and 3 -form flux can be written as

$$
\begin{aligned}
d s^{2} & =\eta_{a b} \lambda^{a} \lambda^{b}+d \tilde{s}_{(7)}^{2}+\mathcal{O}\left(\alpha^{\prime 2}\right), \\
H & =\operatorname{CS}(\lambda)+\tilde{H}_{(7)}+\mathcal{O}\left(\alpha^{\prime 2}\right),
\end{aligned}
$$

where $\operatorname{CS}(\lambda)$ is the Chern-Simons form ${ }^{3}$ of the principal bundle connection,

$$
\lambda^{-}=\mathbf{e}^{-}, \quad \lambda^{+}=\mathbf{e}^{+}-\frac{1}{2} k^{2} u^{2} \mathbf{e}^{-}-u h, \quad \lambda^{1}=k^{-1}\left(h+k^{2} u \mathbf{e}^{-}\right),
$$

$k^{2}=h^{2}$ is constant up to $\mathcal{O}\left(\alpha^{\prime 2}\right)$ and

$$
\tilde{H}_{(7)}=k \varphi+e^{2 \Phi} \star_{7} d\left(e^{-2 \Phi} \varphi\right)+\mathcal{O}\left(\alpha^{\prime 2}\right) .
$$

\footnotetext{
${ }^{3}$ Note that $\operatorname{CS}(\lambda)=d u \wedge d r \wedge h+r d u \wedge d h+k^{-2} h \wedge d h$.
} 
The 3 -form $\varphi$ is the fundamental $G_{2}$ form and it is related to the fundamental $\operatorname{Spin}(7)$ form of the $\eta_{+}$Killing spinor via $\varphi=k^{-1} i_{h} \phi+\mathcal{O}\left(\alpha^{\prime 2}\right)$. The associated vector fields to $\lambda^{-}, \lambda^{+}, \lambda^{1}$ satisfy a $\mathfrak{s l}(2, \mathbb{R})$ algebra. The dilaton $\Phi$ depends only on the coordinates of $B^{7}$.

To find solutions, one has to solve the remaining equations

$$
\begin{aligned}
d\left[e^{-2 \Phi} \star_{7} \varphi\right] & =\mathcal{O}\left(\alpha^{\prime 2}\right), \\
k^{-2} d h \wedge d h+d \tilde{H}_{(7)} & =-\frac{\alpha^{\prime}}{4}\left(-2 d h \wedge d h+\operatorname{tr}\left(\check{R}_{(8)} \wedge \check{R}_{(8)}-F \wedge F\right)\right)+\mathcal{O}\left(\alpha^{\prime 2}\right), \\
(d h)_{i j} & =\frac{1}{2} \star_{7} \varphi_{i j}{ }^{k l}(d h)_{k l}+\mathcal{O}\left(\alpha^{\prime 2}\right), \quad F_{i j}=\frac{1}{2} \star_{7} \varphi_{i j}{ }^{k l} F_{k l}+\mathcal{O}\left(\alpha^{\prime 2}\right) .
\end{aligned}
$$

The first condition in (6.4) is required for $B^{7}$ to admit a $G_{2}$ structure compatible with a connection with skew-symmetric torsion. The second condition is the anomalous Bianchi identity of the 3 -form field strength written in terms of $B^{7}$ data. The curvature $\check{R}_{(8)}$ is that of the near horizon section $\mathcal{S}$ with metric and skew symmetric torsion given by

$$
d \tilde{s}_{(8)}^{2}=k^{-2} h \otimes h+d \tilde{s}_{(7)}^{2}+\mathcal{O}\left(\alpha^{\prime 2}\right), \quad \tilde{H}_{(8)}=k^{-2} h \wedge d h+\tilde{H}_{(7)}+\mathcal{O}\left(\alpha^{\prime 2}\right) .
$$

As $\check{R}_{(8)}$ is invariant under $h$ and $i_{h} \check{R}_{(8)}=\mathcal{O}\left(\alpha^{\prime 2}\right)$, it descends on $B^{7}$. Finally, the last two equations in (6.4) imply that both $d h$ and $F$ are $\mathfrak{g}_{2}$ instantons on $B^{7}$.

\subsection{Horizons with SU(3) structure}

Such horizons preserve 4 supersymmetries. Locally the spacetime is a principal bundle with fibre $\mathrm{SL}(2, \mathbb{R}) \times \mathrm{U}(1)$ over a Kähler with torsion manifold $(\mathrm{KT}) B^{6}$ with Hermitian form $\omega_{(6)}$. The metric and 3 -form field strength of the spacetime can be written as

$$
d s^{2}=\eta_{a b} \lambda^{a} \lambda^{b}+d \tilde{s}_{(6)}^{2}+\mathcal{O}\left(\alpha^{\prime 2}\right), \quad H=\operatorname{CS}(\lambda)+\tilde{H}_{(6)}+\mathcal{O}\left(\alpha^{\prime 2}\right),
$$

where $\lambda^{a}, a=+,-, 1,6$ is the principal bundle connections whose $a=+,-, 1$ components are as in (6.2) and

$$
\lambda^{6}=k^{-1} \ell,
$$

which is along the $\mathfrak{u}(1)$ direction in the Lie algebra. $h^{2}=k^{2}$ is constant up to $\mathcal{O}\left(\alpha^{2}\right)$. The curvature of the principal bundle connection $\lambda^{a}$ is expressed in terms of $d h$ and $d \ell$ which are 2 -forms on $B^{6}$ and it is required to satisfy that

$$
d h^{2,0}=d \ell^{2,0}=\mathcal{O}\left(\alpha^{\prime 2}\right), \quad d h_{i j} \omega_{(6)}^{i j}=\mathcal{O}\left(\alpha^{\prime 2}\right), \quad d \ell_{i j} \omega_{(6)}^{i j}=-2 k^{2}+\mathcal{O}\left(\alpha^{\prime 2}\right),
$$

ie $h$ is a $\mathfrak{s u}(3)$ instanton on $B^{6}$ while $\ell$ is a $\mathfrak{u}(3)$ instanton on $B^{6}$.

The KT manifold $B^{6}$ is in addition conformally balanced, ie

$$
\theta_{\omega_{(6)}}=2 d \Phi+\mathcal{O}\left(\alpha^{2}\right)
$$

where $\theta$ is the Lee form and the torsion is

$$
\tilde{H}_{(6)}=-i_{I} d \omega+\mathcal{O}\left(\alpha^{\prime 2}\right)=e^{2 \Phi} \star_{6} d\left[e^{-2 \Phi} \omega_{(6)}\right]+\mathcal{O}\left(\alpha^{\prime 2}\right) .
$$


The dilaton $\Phi$ depends only on the coordinates of $B^{6}$. The gauge connection is a $\mathfrak{s u}(3)$ instanton on $B^{6}$, i.e.

$$
F^{2,0}=\mathcal{O}\left(\alpha^{\prime}\right), \quad F_{i j} \omega_{(6)}^{i j}=\mathcal{O}\left(\alpha^{\prime}\right) .
$$

To find examples for such horizons two additional conditions should be satisfied. One is the restriction that

$$
\hat{\tilde{R}}_{(6) i j} \omega_{(6)}^{i j}=-2 k^{2} d \ell+\mathcal{O}\left(\alpha^{\prime 2}\right) .
$$

This arises from requirement that the $\mathrm{U}(3)$ structure on $B^{6}$ lifts to a $\mathrm{SU}(3)$ structure on the spacetime or equivalent the spatial horizon section $\mathcal{S}$. The other is the anomalous Bianchi identity which now reads

$$
\begin{aligned}
k^{-2} d h & \wedge d h+k^{-2} d \ell \wedge d \ell+d\left(e^{2 \Phi} \star_{6} d\left[e^{-2 \Phi} \omega\right]\right)= \\
- & \frac{\alpha^{\prime}}{4}\left(-2 d h \wedge d h+\operatorname{tr}\left(\check{R}_{(8)} \wedge \check{R}_{(8)}-F \wedge F\right)\right)+\mathcal{O}\left(\alpha^{\prime 2}\right),
\end{aligned}
$$

where $\check{R}_{(8)}$ is the curvature of the connection with torsion on $\mathcal{S}$ for which the metric and torsion are given by

$$
\begin{aligned}
d \tilde{s}^{2} & =k^{-2}(h \otimes h+\ell \otimes \ell)+d \tilde{s}_{(6)}^{2}+\mathcal{O}\left(\alpha^{\prime 2}\right), \\
\tilde{H} & =k^{-2}(h \wedge d h+\ell \wedge d \ell)+\tilde{H}_{(6)}+\mathcal{O}\left(\alpha^{\prime 2}\right) .
\end{aligned}
$$

Note that $\hat{\nabla}_{(8)}$ has holonomy contained in $\mathrm{SU}(3)$ and so $\check{R}_{(8)}$ is a well defined form on $B^{6}$.

\subsection{Horizons with $\mathrm{SU}(2)$ structure and 6 supersymmetries}

The spacetime is locally a $\mathrm{SL}(2, \mathbb{R}) \times \mathrm{SU}(2)$ principal fibration over a 4-dimensional antiself-dual Weyl Einstein manifold $B^{4}$ with metric $d s_{(4)}^{2}$ and quaternionic Kähler structure 2 -forms $\omega_{(4)}^{r^{\prime}}$. The spacetime metric and 3-form field strength can be expressed as

$$
\begin{aligned}
d s^{2} & =\eta_{a b} \lambda^{a} \lambda^{b}+\delta_{r^{\prime} s^{\prime}} \lambda^{r^{\prime}} \lambda^{s^{\prime}}+e^{2 \Phi} d s_{(4)}^{2}+\mathcal{O}\left(\alpha^{\prime 2}\right), \\
H & =\operatorname{CS}(\lambda)+\tilde{H}_{(4)}+\mathcal{O}\left(\alpha^{\prime 2}\right),
\end{aligned}
$$

where $\tilde{H}_{(4)}=-\stackrel{\circ}{\star} 4_{4} d e^{2 \Phi}$, the principal bundle connection $\lambda^{a}$ for $a=+,-, 1$ coincides with that of (6.2) while

$$
\lambda^{r^{\prime}}=k^{-1} \ell^{r^{\prime}},
$$

are the components along the $\mathfrak{s u}(2)$ subalgebra of the fibre. Furthermore the dilaton depends only on the coordinates of $B^{4}$, dh as well as the curvature $\left(\mathcal{F}^{\text {sd }}\right)^{r^{\prime}}$ of $\lambda^{r^{\prime}}$ are 2 -forms on $B^{4}$. In addition, we have that

$$
d h^{\mathrm{sd}}=\mathcal{O}\left(\alpha^{\prime 2}\right), \quad\left(\mathcal{F}^{\mathrm{sd}}\right)^{r^{\prime}}=\frac{k}{4} \omega_{(4)}^{r^{\prime}}+\mathcal{O}\left(\alpha^{\prime 2}\right), \quad F^{\mathrm{sd}}=\mathcal{O}\left(\alpha^{\prime}\right)
$$

and $d h^{\text {ad }},\left(\mathcal{F}^{\text {ad }}\right)^{r^{\prime}}$ and $F^{\text {ad }}$ are not restricted, where the self-dual and anti-self dual components are appropriately denoted. Geometrically, the set up is such that the $\mathrm{SO}(4)=$ $\mathrm{SU}(2) \cdot \mathrm{SU}(2)$ structure of $B^{4}$ when lifted the 7 -dimensional manifold which is the principal bundle with fibre $\mathrm{SU}(2)$ reduces to $\mathrm{SU}(2)$ as required from supersymmetry. 
The only remaining condition to find solutions is

$$
\begin{aligned}
& \stackrel{\circ}{\nabla}^{2} e^{2 \Phi}=-\frac{1}{2}\left(\mathcal{F}^{\mathrm{ad}}\right)_{i j}^{r^{\prime}}\left(\mathcal{F}^{\mathrm{ad}}\right)_{r^{\prime}}^{i j}-\frac{k^{-2}}{2} d h_{i j} d h^{i j}+\frac{3}{8} k^{2} e^{4 \Phi} \\
& +\frac{\alpha^{\prime}}{8}\left(-2 d h_{i j} d h^{i j}+\operatorname{tr}\left(\check{R}_{(8) i j} \check{R}_{(8)}{ }^{i j}-F_{i j} F^{i j}\right)\right)+\mathcal{O}\left(\alpha^{\prime 2}\right) \text {. }
\end{aligned}
$$

Again $\check{R}_{(8)}$ is the curvature of the connection with torsion of the horizon section $\mathcal{S}$ which has metric and 3 -form field strength

$$
\begin{aligned}
d \tilde{s}^{2} & =k^{-2} h \otimes h+\delta_{r^{\prime} s^{\prime}} \lambda^{r^{\prime}} \lambda^{s^{\prime}}+e^{2 \Phi} d \stackrel{\circ}{(4)}_{(4)}^{2}+\mathcal{O}\left(\alpha^{\prime 2}\right), \\
\tilde{H} & =k^{-2} h \wedge d h+\operatorname{CS}\left(\lambda^{r^{\prime}}\right)+\tilde{H}_{(4)}+\mathcal{O}\left(\alpha^{\prime 2}\right) .
\end{aligned}
$$

As $\hat{R}_{(8)}$ has holonomy contained in $\mathrm{SU}(2), \check{R}_{(8)}$ is a 2 -form on $B^{4}$. For more details on the geometry of heterotic backgrounds that preserve 6 supersymmetries and have $\mathrm{SU}(2)$ holonomy see [27, 28].

\subsection{Horizons with $\mathrm{SU}(2)$ structure and 8 supersymmetries}

This class of horizons have a similar geometry to those of the previous section that preserve 6 supersymmetries. The differences are that

$$
\left(\mathcal{F}^{\mathrm{sd}}\right)^{r^{\prime}}=\mathcal{O}\left(\alpha^{\prime 2}\right)
$$

so $\mathcal{F}^{r^{\prime}}$ is an anti-self dual instanton on $B^{4}$ which now is a hyper-Kähler manifold with respect to the metric $d s_{(4)}^{2}$. Furthermore the equation for the dilaton (6.18) now reads

$$
\begin{aligned}
\stackrel{\circ}{\nabla}^{2} e^{2 \Phi}= & -\frac{1}{2} \mathcal{F}_{i j}^{r^{\prime}} \mathcal{F}_{r^{\prime}}^{i j}-\frac{k^{-2}}{2} d h_{i j} d h^{i j} \\
& +\frac{\alpha^{\prime}}{8}\left(-2 d h_{i j} d h^{i j}+\operatorname{tr}\left(\check{R}_{(8) i j} \check{R}_{(8)}{ }^{i j}-F_{i j} F^{i j}\right)\right)+\mathcal{O}\left(\alpha^{\prime 2}\right) .
\end{aligned}
$$

Therefore at zeroth order, a partial integration argument reveals that

$$
d h=\mathcal{O}\left(\alpha^{\prime}\right), \quad \mathcal{F}^{r^{\prime}}=\mathcal{O}\left(\alpha^{\prime}\right) .
$$

Thus $B^{4}$ up to a local isometry is $\mathrm{AdS}_{3} \times S^{3} \times T^{4}$ or $\mathrm{AdS}_{3} \times S^{3} \times K_{3}$ and the dilaton is constant. One does not expect additional $\alpha^{\prime}$ corrections to the geometry in the case that the $\check{R}_{(8)}$ is identified with $F$. Though additional corrections are expected otherwise. In the absence of 5 -branes, consistency requires that the Pontryagin number of the tangent bundle of $B^{4}$ cancels that of the gauge bundle which is the vanishing condition for the global anomaly.

\section{Global properties}

\subsection{Maximum principle on $h^{2}$}

We shall revisit the global analysis of [27] by calculating the Laplacian of $h^{2}$, but including also $\alpha^{\prime}$ correction terms. Then we shall examine the conditions imposed on the geometry by this expression. To avoid the trivial case when $h^{2}=\mathcal{O}\left(\alpha^{\prime 2}\right)$, we take $h^{[0]} \neq 0$. 
Next we calculate the Laplacian of $h^{2}$ to find that

$$
\begin{aligned}
\tilde{\nabla}^{i} \tilde{\nabla}_{i} h^{2}+(h-2 d \Phi)^{j} \tilde{\nabla}_{j} h^{2}= & 2 \tilde{\nabla}^{(i} h^{j)} \tilde{\nabla}_{(i} h_{j}+\frac{1}{2}\left(d h-i_{h} W\right)_{i j}\left(d h-i_{h} W\right)^{i j} \\
& -\frac{\alpha^{\prime}}{4} h^{i} h^{j}\left(-2 d h_{i \ell} d h_{j}^{\ell}+\check{\tilde{R}}_{i \ell_{1} \ell_{2} \ell_{3}} \check{\tilde{R}}_{j}^{\ell_{1} \ell_{2} \ell_{3}}-\tilde{F}_{i \ell}{ }^{a b} \tilde{F}_{j}^{\ell}{ }_{a b}\right)+\mathcal{O}\left(\alpha^{\prime 2}\right) .
\end{aligned}
$$

In computing this expression, we made use of the Einstein equation (4.16) together with the gauge field equations (4.13) and (4.14). We remark that the calculation proceeds in exactly the same way as in [27]; the $\alpha^{\prime}$ terms in (7.1) originate from the $\alpha^{\prime}$ terms in $2 h^{i} h^{j} \tilde{R}_{i j}$. It should be noted that in order to fully control $\mathcal{O}\left(\alpha^{\prime 2}\right)$ terms in this expression, one would require to know the Einstein equations up to and including $\alpha^{\prime 2}$.

To begin, we consider (7.1) to zeroth order in $\alpha^{\prime}$. We then re-obtain the conditions found in [27] via a maximum principle argument, i.e.

$$
h^{2}=\text { const }+\mathcal{O}\left(\alpha^{\prime}\right), \quad \tilde{\nabla}_{(i} h_{j)}=\mathcal{O}\left(\alpha^{\prime}\right), \quad d h-i_{h} W=\mathcal{O}\left(\alpha^{\prime}\right) .
$$

In particular, it follows from these conditions that

$$
i_{h} d h=O\left(\alpha^{\prime}\right)
$$

and also

$$
\mathcal{L}_{h} \Phi=\mathcal{O}\left(\alpha^{\prime}\right), \quad \mathcal{L}_{h} W=\mathcal{O}\left(\alpha^{\prime}\right) .
$$

Furthermore, it also follows that if $\eta_{+}$satisfies (4.26), then $\Gamma_{-} h_{i} \Gamma^{i} \eta_{+}$also satisfies (4.26) to zeroth order in $\alpha^{\prime}$. The integrability conditions therefore imply that

$$
\hat{\tilde{R}}_{i j m n} h^{m} \Gamma^{n} \phi_{+}=\mathcal{O}\left(\alpha^{\prime}\right),
$$

and hence

$$
\check{\tilde{R}}_{m n i j} h^{m}=\mathcal{O}\left(\alpha^{\prime}\right) .
$$

On substituting these conditions back into (7.1) one finds that the remaining content of $(7.1)$ is

$$
\tilde{\nabla}^{i}\left(e^{-2 \Phi} \tilde{\nabla}_{i} h^{2}\right)+e^{-2 \Phi} h^{j} \tilde{\nabla}_{j} h^{2}=\frac{\alpha^{\prime}}{2} e^{-2 \Phi} h^{i} h^{j} \tilde{F}_{i \ell}^{a b} \tilde{F}_{j}^{\ell} a b+\mathcal{O}\left(\alpha^{\prime 2}\right) .
$$

On integrating the $\mathcal{O}\left(\alpha^{\prime}\right)$ part of (7.7) over the zeroth order horizon section, one finds that

$$
i_{h} \tilde{F}=\mathcal{O}\left(\alpha^{\prime}\right),
$$

and furthermore

$$
h^{2}=\text { const }+\mathcal{O}\left(\alpha^{\prime 2}\right) .
$$

It should be noted however that (7.1) does not in general imply (5.1). In particular, the conditions obtained from the analysis of the properties of $h^{2}$ are not sufficient to imply that if $\eta_{+}$, with $\eta_{+}^{[0]} \neq 0$, satisfies (4.26) and (4.27), then $\eta_{-}^{\prime \prime}=\Gamma_{-} h_{i} \Gamma^{i} \eta_{+}$also satisfies (4.26) and (4.27). Thus although (7.1) implies the horizons exhibit supersymmetry enhancement at $\mathcal{O}\left(\alpha^{\prime}\right)$, it does not imply the same at $\mathcal{O}\left(\alpha^{\prime 2}\right)$. 


\subsection{Lichnerowicz type theorems}

Next we shall investigate whether it is possible to identify Killing spinors with the zero modes of a suitable Dirac-like operator, by constructing a generalized Lichnerowicz type theorem which incorporates the near-horizon fluxes. Such Lichnerowicz type theorems have been established for near-horizon geometries in $\mathrm{D}=11$ supergravity [9], type IIB [10] and type IIA supergravity (both massive and massless) [11, 12], as well as for AdS geometries in ten and eleven dimensional supergravity [33, 37-39].

To begin, let us first define the modified connection with torsion and the modified horizon Dirac operator, respectively

$$
\nabla_{i}^{(\kappa)} \equiv \hat{\tilde{\nabla}}_{i}+\kappa \Gamma_{i} \mathcal{A}, \quad \mathcal{D} \equiv \Gamma^{i} \hat{\tilde{\nabla}}_{i}+q \mathcal{A},
$$

where $\kappa, q \in \mathbb{R}$, and

$$
\begin{aligned}
\hat{\tilde{\nabla}}_{i} \eta_{ \pm} & =\tilde{\nabla}_{i} \eta_{ \pm}-\frac{1}{8} W_{i j k} \Gamma^{j k} \eta_{ \pm} \\
\mathcal{A} & =W_{i j k} \Gamma^{i j k}-12 \Gamma^{i} \tilde{\nabla}_{i} \Phi \mp 6 \Gamma^{i} h_{i} .
\end{aligned}
$$

It is clear that if $\eta_{ \pm}$is a Killing spinor, i.e.

$$
\hat{\tilde{\nabla}}_{i} \eta_{ \pm}=\mathcal{O}\left(\alpha^{\prime 2}\right), \quad \text { and } \quad \mathcal{A} \eta_{ \pm}=\mathcal{O}\left(\alpha^{\prime 2}\right),
$$

then $\mathcal{D} \eta_{ \pm}=\mathcal{O}\left(\alpha^{\prime 2}\right)$ also. Here we want to investigate the extent to which the converse is true. We shall show that if $\mathcal{D} \eta_{ \pm}=\mathcal{O}\left(\alpha^{\prime 2}\right)$, then

$$
\hat{\tilde{\nabla}}_{i} \eta_{ \pm}=\mathcal{O}\left(\alpha^{\prime}\right), \quad \text { and } \quad \mathcal{A} \eta_{ \pm}=\mathcal{O}\left(\alpha^{\prime}\right)
$$

and moreover

$$
d h_{i j} \Gamma^{i j} \eta_{ \pm}=\mathcal{O}\left(\alpha^{\prime}\right), \quad \text { and } \quad \tilde{F}_{i j}^{a b} \Gamma^{i j} \eta_{ \pm}=\mathcal{O}\left(\alpha^{\prime}\right) .
$$

In order to obtain this result, we begin by considering the following functional

$$
\mathcal{I} \equiv \int_{\mathcal{S}} e^{c \Phi}\left(\left\langle\nabla_{i}^{(\kappa)} \eta_{ \pm}, \nabla^{(\kappa) i} \eta_{ \pm}\right\rangle-\left\langle\mathcal{D} \eta_{ \pm}, \mathcal{D} \eta_{ \pm}\right\rangle\right)
$$

where $c \in \mathbb{R}$, and we assume all the field equations. After some algebra, which is described in appendix D, we find

$$
\begin{aligned}
\mathcal{I}= & \left(8 \kappa^{2}-\frac{1}{6} \kappa\right) \int_{\mathcal{S}} e^{-2 \Phi}\left\|\mathcal{A} \eta_{ \pm}\right\|^{2}+\int_{\mathcal{S}} e^{-2 \Phi}\left\langle\eta_{ \pm}, \Psi \mathcal{D} \eta_{ \pm}\right\rangle \\
& -\frac{\alpha^{\prime}}{64} \int_{\mathcal{S}} e^{-2 \Phi}\left(2\left\|d / h \eta_{ \pm}\right\|^{2}+\left\|\tilde{F} \eta_{ \pm}\right\|^{2}-\left\langle\check{\tilde{R}}_{\ell_{1} \ell_{2}, i j} \Gamma^{\ell_{1} \ell_{2}} \eta_{ \pm}, \check{\tilde{R}}_{\ell_{3} \ell_{4}}^{i j}, \Gamma^{\ell_{3} \ell_{4}} \eta_{ \pm}\right\rangle\right)+\mathcal{O}\left(\alpha^{\prime 2}\right),
\end{aligned}
$$

which is true if and only if $q=\frac{1}{12}+\mathcal{O}\left(\alpha^{\prime 2}\right)$ and $c=-2+\mathcal{O}\left(\alpha^{\prime 2}\right)$, and the $\Psi$ is defined as follows

$$
\Psi \equiv 2\left(\kappa-\frac{1}{12}\right) \mathcal{A}^{\dagger}-2 \Gamma^{i} \tilde{\nabla}_{i} \Phi-\frac{1}{6} \Gamma^{\ell_{1} \ell_{2} \ell_{3}} W_{\ell_{1} \ell_{2} \ell_{3}}+\mathcal{O}\left(\alpha^{\prime 2}\right)
$$


The values of $q$ and $c$ are fixed by requiring that certain terms in the functional (7.15), which cannot be rewritten in terms of the Dirac operator $\mathcal{D}$, or $\mathcal{A}^{\dagger} \mathcal{A}$, and which have no fixed sign, should vanish.

The part of (7.16) which is of zeroth order in $\alpha^{\prime}$ implies that if $0<\kappa<\frac{1}{48}$, then

$$
\mathcal{D} \eta_{ \pm}=\mathcal{O}\left(\alpha^{\prime 2}\right) \quad \Longrightarrow(7.13)
$$

and establishes the first part of the theorem. Next the integrability condition of $\hat{\tilde{\nabla}} \eta_{ \pm}=\mathcal{O}\left(\alpha^{\prime}\right)$ is

$$
\hat{\tilde{R}}_{m n, \ell_{1} \ell_{2}} \Gamma^{\ell_{1} \ell_{2}} \eta_{ \pm}=\mathcal{O}\left(\alpha^{\prime}\right)
$$

which in turn implies that

$$
\check{\tilde{R}}_{\ell_{1} \ell_{2}, m n} \Gamma^{\ell_{1} \ell_{2}} \eta_{ \pm}=\mathcal{O}\left(\alpha^{\prime}\right)
$$

Hence we shall neglect the term in (7.16) which is quadratic in $\tilde{\tilde{R}}$, as this term is $\mathcal{O}\left(\alpha^{\prime 3}\right)$. Then, assuming (7.18), the part of (7.16) which is first order in $\alpha^{\prime}$ further implies (7.14). This completes the proof.

\section{Nearly supersymmetric horizons}

\subsection{Description of the backgrounds}

We have proven that for near horizon geometries the necessary and sufficient conditions imposed by supersymmetry on the spinors can be reduced to (4.26) and (4.27). In this section, we shall consider the case for which the supersymmetry is explicitly partially broken, in the sense that the gravitino KSE (4.26) admits solutions but not dilatino one (4.27). We also assume that the fields satisfy

$$
\Delta=\mathcal{O}\left(\alpha^{\prime 2}\right), \quad H=d\left(\mathbf{e}^{-} \wedge \mathbf{e}^{+}\right)+W+\mathcal{O}\left(\alpha^{\prime 2}\right) .
$$

These conditions were previously obtained via the supersymmetry analysis; here we shall assume them. In particular, all of the conditions obtained from the global analysis of the Laplacian of $h^{2}$ in section 7 remain true. As a consequence of this,

$$
\hat{\tilde{\nabla}}_{i} h_{j}=\mathcal{O}\left(\alpha^{\prime}\right)
$$

However we do not assume that $\hat{\tilde{\nabla}} h=\mathcal{O}\left(\alpha^{2}\right)$.

One consequence of these assumptions is that none of the spacetime Killing spinor equations are satisfied even at $\mathcal{O}\left(\alpha^{\prime}\right)$. In particular, the spacetime gravitino KSE requires in addition the condition that $d h_{i j} \Gamma^{i j} \eta_{+}=\mathcal{O}\left(\alpha^{\prime}\right)$ which is not one of our requirements. In what follows, we shall investigate the consequences of the above assumptions on the geometry of the spatial horizon sections $\mathcal{S}$. We shall also comment on the special case where $\hat{\tilde{\nabla}} h=\mathcal{O}\left(\alpha^{\prime 2}\right)$. 


\subsection{Additional parallel spinors}

A key property of backgrounds that satisfy the gravitino KSE but not the dilatino one is the existence of additional parallel spinors, see also appendix C. In the present context to show this focus on the spinor $\eta_{+}$; a similar analysis can be undertaken for the $\eta_{-}$spinors. To proceed, it will be useful to define

$$
\mathcal{A}=W_{i j k} \Gamma^{i j k}-12 \Gamma^{i} \tilde{\nabla}_{i} \Phi-6 h_{i} \Gamma^{i},
$$

so that the algebraic condition (4.27) on $\eta_{+}$is equivalent to $\mathcal{A} \eta_{+}=\mathcal{O}\left(\alpha^{\prime 2}\right)$. We then note the useful identity

$$
\begin{aligned}
\tilde{\nabla}_{i} W_{\ell_{1} \ell_{2} \ell_{3}} \Gamma^{\ell_{1} \ell_{2} \ell_{3}} \eta_{+}= & \tilde{\nabla}_{i}\left(\mathcal{A} \eta_{+}\right)-\frac{1}{8} W_{i \ell_{1} \ell_{2}} \Gamma^{\ell_{1} \ell_{2}}\left(\mathcal{A} \eta_{+}\right) \\
& +3 W_{\ell_{1} \ell_{2} q} W_{i \ell_{3}} \Gamma^{\ell_{1} \ell_{2} \ell_{3}} \eta_{+}-\left(6 \tilde{\nabla}^{m} \Phi+3 h^{m}\right) W_{m i \ell} \Gamma^{\ell} \eta_{+} \\
& +\left(12 \Gamma^{\ell} \tilde{\nabla}_{i} \tilde{\nabla}_{\ell} \Phi+6 \tilde{\nabla}_{i} h_{\ell} \Gamma^{\ell}\right) \eta_{+} .
\end{aligned}
$$

The integrability conditions of (4.26) imply that

$$
\begin{aligned}
\frac{1}{6}\left(\tilde{\nabla}_{i}\left(\mathcal{A} \eta_{+}\right)-\frac{1}{8} W_{i \ell_{1} \ell_{2}} \Gamma^{\ell_{1} \ell_{2}}\left(\mathcal{A} \eta_{+}\right)\right) & -\frac{\alpha^{\prime}}{8}\left(\tilde{F}_{i \ell}\right)_{a b} \Gamma^{\ell}\left(\tilde{F}_{q_{1} q_{2}}\right)^{a b} \Gamma^{q_{1} q_{2}} \eta_{+} \\
& -\frac{\alpha^{\prime}}{16} d h_{i \ell} \Gamma^{\ell} d h_{q_{1} q_{2}} \Gamma^{q_{1} q_{2}} \eta_{+}=\mathcal{O}\left(\alpha^{\prime 2}\right),
\end{aligned}
$$

and hence

$$
\begin{aligned}
\frac{1}{6}\left\langle\eta_{+},\right. & \left.\Gamma^{i} \tilde{\nabla}_{i}\left(\mathcal{A} \eta_{+}\right)-\frac{1}{8} W_{\ell_{1} \ell_{2} \ell_{3}} \Gamma^{\ell_{1} \ell_{2} \ell_{3}}\left(\mathcal{A} \eta_{+}\right)\right\rangle+\frac{\alpha^{\prime}}{8}\left\langle\left(\left(\tilde{F}_{\ell_{1} \ell_{2}}\right)_{a b} \Gamma^{\ell_{1} \ell_{2}} \eta_{+},\left(\tilde{F}_{q_{1} q_{2}}\right)^{a b} \Gamma^{q_{1} q_{2}} \eta_{+}\right\rangle\right. \\
+ & \frac{\alpha^{\prime}}{16}\left\langle d h_{\ell_{1} \ell_{2}} \Gamma^{\ell_{1} \ell_{2}} \phi_{+}, d h_{q_{1} q_{2}} \Gamma^{q_{1} q_{2}} \eta_{+}\right\rangle=\mathcal{O}\left(\alpha^{\prime 2}\right) .
\end{aligned}
$$

Integrating this expression over $\mathcal{S}$ yields the conditions

$$
\tilde{F}_{i j} \Gamma^{i j} \eta_{+}=\mathcal{O}\left(\alpha^{\prime}\right), \quad d h_{i j} \Gamma^{i j} \eta_{+}=\mathcal{O}\left(\alpha^{\prime}\right),
$$

and substituting these conditions back into (8.5) then implies that

$$
\tilde{\nabla}_{i}\left(\mathcal{A} \eta_{+}\right)-\frac{1}{8} W_{i \ell_{1} \ell_{2}} \Gamma^{\ell_{1} \ell_{2}}\left(\mathcal{A} \eta_{+}\right)=\mathcal{O}\left(\alpha^{\prime 2}\right) .
$$

Therefore the spinor $\tau_{+}=\mathcal{A} \eta_{+}$is also $\hat{\tilde{\nabla}}$-parallel. As $\tau_{+}$has opposite chirality from $\eta_{+}$ cannot be identified as an additional Killing spinor within the heterotic theory. Nevertheless it is instrumental in the description of the geometry of $\mathcal{S}$.

\subsection{Nearly supersymmetric horizons with $G_{2}$ holonomy}

\subsubsection{A symmetry of horizon section}

Suppose that we consider solutions for which there exists a single solution $\eta_{+}$to the gravitino KSE

$$
\hat{\tilde{\nabla}} \eta_{+}=\mathcal{O}\left(\alpha^{\prime 2}\right)
$$


for which $\left(\mathcal{A} \eta_{+}\right)^{[0]} \neq 0$. This implies that the horizon section $\mathcal{S}^{[0]}$ at zeroth order in $\alpha^{\prime}$ admits a $G_{2}$ structure.

We begin by defining $\tau_{+}=\mathcal{A} \eta_{+}$, with $\tau_{+}^{[0]} \neq 0$. It will be particularly useful to define

$$
V_{i}=\left\langle\eta, \Gamma_{i} \tau_{+}\right\rangle
$$

In what follows we shall show that $V$ is a symmetry of all the fields of the spatial horizon section.

As $\tau_{+}^{[0]} \neq 0$, this implies that $V^{[0]} \neq 0$. In addition, as $\eta_{+}$and $\tau_{+}$satisfy

$$
\hat{\tilde{\nabla}} \eta_{+}=\mathcal{O}\left(\alpha^{\prime 2}\right), \quad \hat{\tilde{\nabla}} \tau_{+}=\mathcal{O}\left(\alpha^{\prime 2}\right),
$$

it follows that

$$
\hat{\tilde{\nabla}} V=\mathcal{O}\left(\alpha^{2}\right)
$$

so that $V^{2}=$ const. $+\mathcal{O}\left(\alpha^{2}\right)$, and $V$ is an isometry of $\mathcal{S}$ to both zero and first order in $\alpha^{\prime}$.

Next, we consider the relationship of $V$ to $h$. In particular, the spinors $h_{i} \Gamma^{i} \mathcal{A} \eta_{+}$and $V_{i} \Gamma^{i} \mathcal{A} \eta_{+}$are both parallel with respect to $\hat{\tilde{\nabla}}$ at zeroth order in $\alpha^{\prime}$. As we have assumed that (8.9) admits only one solution, there must be a nonzero constant $c$ such that

$$
V=\operatorname{ch}+\mathcal{O}\left(\alpha^{\prime}\right)
$$

In addition, we have

$$
\mathcal{L}_{V} W=i_{V} d W+\mathcal{O}\left(\alpha^{\prime 2}\right),
$$

because $d V=i_{V} W+\mathcal{O}\left(\alpha^{\prime 2}\right)$. Also, as $V=c h+\mathcal{O}\left(\alpha^{\prime}\right)$ it follows that

$$
\mathcal{L}_{V} W=c i_{h} d W+\mathcal{O}\left(\alpha^{\prime 2}\right)
$$

As a consequence of (8.2), one has that $i_{h} d h=\mathcal{O}\left(\alpha^{\prime}\right)$, and from the global analysis of the Laplacian of $h^{2}$, we find $i_{h} \tilde{F}=\mathcal{O}\left(\alpha^{\prime}\right)$ as well as $\check{\tilde{R}}_{m n i j} h^{m}=\mathcal{O}\left(\alpha^{\prime}\right)$. These conditions imply that

$$
\mathcal{L}_{V} W=\mathcal{O}\left(\alpha^{\prime 2}\right)
$$

and so $W$ is invariant.

Next we consider $\mathcal{L}_{V} \Phi$. As $V=c h+\mathcal{O}\left(\alpha^{\prime}\right)$ it follows that

$$
\mathcal{L}_{V} d h=c \mathcal{L}_{h} d h+\mathcal{O}\left(\alpha^{\prime}\right)=\mathcal{O}\left(\alpha^{\prime}\right) .
$$

Also we have

$$
\mathcal{L}_{V} \tilde{R}_{i j, p q}=\mathcal{O}\left(\alpha^{\prime 2}\right),
$$

and

$$
\left(\mathcal{L}_{V} \tilde{F}\right)_{i j}{ }_{b b} \tilde{F}^{i j b}{ }_{a}=\mathcal{O}\left(\alpha^{\prime}\right),
$$

which follows from

$$
\mathcal{L}_{V} \tilde{F}=c\left[\tilde{F}, i_{h} \mathcal{B}\right]+\mathcal{O}\left(\alpha^{\prime}\right)
$$


Hence we have

$$
\mathcal{L}_{V}\left(\alpha^{\prime}\left(-2 d h_{i j} d h^{i j}+\check{\tilde{R}}_{i j, p q} \check{\tilde{R}}^{i j, p q}-\left(\tilde{F}_{i j}\right)^{a b}\left(\tilde{F}^{i j}\right)_{a b}\right)\right)=\mathcal{O}\left(\alpha^{\prime 2}\right) .
$$

So, on taking the Lie derivative of the trace of (4.16) with respect to $V$ we find

$$
\mathcal{L}_{V}\left(\tilde{\nabla}^{i} h_{i}+2 \tilde{\nabla}_{i} \tilde{\nabla}^{i} \Phi\right)=\mathcal{O}\left(\alpha^{\prime 2}\right)
$$

and hence, as a consequence of the field equation (4.13), we find

$$
\mathcal{L}_{V}\left(h^{i} \tilde{\nabla}_{i} \Phi+\tilde{\nabla}^{i} \tilde{\nabla}_{i} \Phi\right)=\mathcal{O}\left(\alpha^{\prime 2}\right) .
$$

Also, on taking the Lie derivative of the dilaton field equation (4.17), we get

$$
\mathcal{L}_{V}\left(-h^{i} \tilde{\nabla}_{i} \Phi-2 \tilde{\nabla}_{i} \Phi \tilde{\nabla}^{i} \Phi+\tilde{\nabla}^{i} \tilde{\nabla}_{i} \Phi\right)=\mathcal{O}\left(\alpha^{\prime 2}\right) .
$$

On taking the sum of (8.23) and (8.24), we find

$$
\mathcal{L}_{V}\left(\tilde{\nabla}^{i} \tilde{\nabla}_{i} \Phi-\tilde{\nabla}^{i} \Phi \tilde{\nabla}_{i} \Phi\right)=\mathcal{O}\left(\alpha^{\prime 2}\right)
$$

and hence if $f=\mathcal{L}_{V} \Phi$ we have

$$
\tilde{\nabla}_{i} \tilde{\nabla}^{i} f-2 \tilde{\nabla}^{i} \Phi \tilde{\nabla}_{i} f=\mathcal{O}\left(\alpha^{2}\right) .
$$

We know $\mathcal{L}_{h} \Phi=\mathcal{O}\left(\alpha^{\prime}\right)$ as a consequence of the analysis of the Laplacian of $h^{2}$, so $f=$ $\alpha^{\prime} f^{[1]}+\mathcal{O}\left(\alpha^{\prime 2}\right)$. Then, on integrating, (8.26) implies that

$$
\int_{\mathcal{S}^{[0]}} e^{-2 \Phi^{[0]}} \tilde{\nabla}_{i} f^{[1]} \tilde{\nabla}^{i} f^{[1]}=0
$$

so $f^{[1]}=\beta$ for constant $\beta$, and so

$$
\mathcal{L}_{V} \Phi=\beta \alpha^{\prime}+\mathcal{O}\left(\alpha^{\prime 2}\right)
$$

As we require that $\Phi$ must attain a global maximum on $\mathcal{S}$, at this point $\mathcal{L}_{V} \Phi=0$ to all orders in $\alpha^{\prime}$, for any $V$. This fixes $\beta=0$, so

$$
\mathcal{L}_{V} \Phi=\mathcal{O}\left(\alpha^{\prime 2}\right)
$$

which proves the invariance of $\Phi$.

Next, we consider $\mathcal{L}_{V} h$. On taking the Lie derivative of the field equation of the 2-form gauge potential (4.15) we find

$$
d\left(\mathcal{L}_{V} h\right)_{i j}-\left(\mathcal{L}_{V} h\right)^{k} W_{i j k}=\mathcal{O}\left(\alpha^{\prime 2}\right)
$$

and on taking the Lie derivative of the Einstein equation (4.16) we get

$$
\tilde{\nabla}_{(i}\left(\mathcal{L}_{V} h\right)_{j)}=\mathcal{O}\left(\alpha^{\prime 2}\right),
$$


where we have used

$$
\mathcal{L}_{h}\left(\tilde{F}_{i \ell}^{a b} \tilde{F}_{j}^{\ell} a b\right)=\mathcal{O}\left(\alpha^{\prime}\right)
$$

It follows that

$$
\hat{\tilde{\nabla}}\left(\mathcal{L}_{V} h\right)_{j}=\mathcal{O}\left(\alpha^{\prime 2}\right)
$$

As $V=c h+\mathcal{O}\left(\alpha^{\prime}\right)$, it is convenient to write

$$
\mathcal{L}_{V} h=\alpha^{\prime} \Lambda+\mathcal{O}\left(\alpha^{\prime 2}\right),
$$

where

$$
\hat{\tilde{\nabla}} \Lambda=\mathcal{O}\left(\alpha^{\prime}\right) .
$$

As $\Lambda_{j} \Gamma^{j} \mathcal{A} \eta_{+}$and $h_{j} \Gamma^{j} \mathcal{A} \eta_{+}$are both parallel with respect to $\hat{\tilde{\nabla}}$ at zeroth order in $\alpha^{\prime}$, it follows as a consequence of (ii) that we must have

$$
\Lambda=b h+\mathcal{O}\left(\alpha^{\prime}\right),
$$

for constant $b$. It is also useful to compute

$$
h^{i}\left(\mathcal{L}_{V} h_{i}\right)=h^{i}\left(V^{j} \tilde{\nabla}_{j} h_{i}+h_{j} \tilde{\nabla}_{i} V^{j}\right)=\frac{1}{2} \mathcal{L}_{V} h^{2}+h^{i} h^{j} \tilde{\nabla}_{i} V_{j}=\mathcal{O}\left(\alpha^{\prime 2}\right),
$$

which follows because $h^{2}=$ const $+\mathcal{O}\left(\alpha^{\prime 2}\right)$, and $\hat{\tilde{\nabla}} V=\mathcal{O}\left(\alpha^{\prime 2}\right)$. This implies that $b=0$, and hence

$$
\mathcal{L}_{V} h=\mathcal{O}\left(\alpha^{\prime 2}\right) .
$$

So $V$ is a symmetry of the full solution to both zeroth and first order in $\alpha^{\prime}$.

\subsubsection{Geometry}

We have shown that $V$ is a symmetry of the backgrounds up $\mathcal{O}\left(\alpha^{\prime 2}\right)$. To investigate further the geometry of the horizon section $\mathcal{S}$, let us first consider the consequences of the existence of the $\eta_{+}$Killing spinor. As the isotropy group of $\eta_{+}$in $\operatorname{Spin}(8)$ is $\operatorname{Spin}(7)$, the fundamental self-dual 4 -form $\phi$ of $\operatorname{Spin}(7)$ on $\mathcal{S}$ is $\hat{\tilde{\nabla}}$-parallel. It is known that in such a case, the torsion 3 -form $W$ can be uniquely determined in terms of $\phi$ and the metric without any additional conditions on the $\operatorname{Spin}(7)$ structure of $\mathcal{S}[40]$. Next the condition $\hat{\tilde{\nabla}} \tau_{+}=\mathcal{O}\left(\alpha^{2}\right)$ with $\tau_{+}=\mathcal{A} \eta_{+}$is equivalent to requiring that

$$
\hat{\tilde{\nabla}}_{i}\left((2 d \Phi+h)_{j}-\left(\theta_{\phi}\right)_{j}\right)=\mathcal{O}\left(\alpha^{\prime 2}\right),
$$

where $\theta_{\phi}$ is the Lee form of $\phi$, see [25]. As a result $2 d \Phi+h-\theta_{\phi}$ is a parallel 1-form. If it is not linearly dependent on $V$, it will give rise to an additional solution for the gravitino $\mathrm{KSE} \mathcal{S}$. As we have assumed that there is strictly one parallel spinor of the same chirality as $\eta_{+}$, we have to require that

$$
2 d \Phi+h-\theta_{\phi}=\lambda V+\mathcal{O}\left(\alpha^{\prime 2}\right),
$$

for some non-zero constant $\lambda$; for $\lambda=0$ the dilatino KSE is satisfied as well. 
Let us next turn to investigate the $G_{2}$ structure on $\mathcal{S}$. As $V$ is an isometry on $\mathcal{S}$ and $i_{V} W=d V$, setting $V^{2}=\ell^{2}+\mathcal{O}\left(\alpha^{\prime 2}\right)$ for $\ell$ constant, we can decompose the metric and 3 -form as

$$
d \tilde{s}^{2}=\frac{1}{\ell^{2}} V \otimes V+d s_{(7)}^{2}+\mathcal{O}\left(\alpha^{\prime 2}\right), \quad W=\ell^{-2} V \wedge d V+W_{(7)}+\mathcal{O}\left(\alpha^{\prime 2}\right),
$$

where $d s_{(7)}^{2}$ is the metric on the space orthogonal to $V$ and $i_{V} W_{(7)}=0$. The data $\left(d s_{(7)}^{2}, W_{(7)}\right)$ are thought (locally) as the metric torsion on the space of orbits $M^{7}$ of $V$. For this observe that $\mathcal{L}_{V} W_{(7)}=0$ and as $i_{V} W_{(7)}=0, W_{(7)}$ descends as a 3 -form on the space of orbits.

The spatial horizon section $\mathcal{S}$ admits a $G_{2}$ structure with fundamental form $\varphi=\ell^{-1} i_{V} \phi$ as $\hat{\tilde{\nabla}} \varphi=\mathcal{O}\left(\alpha^{\prime 2}\right)$. The question is whether this $G_{2}$ structure descends on the space of orbits of $V$. First observe that $i_{V} \varphi=0$. So it remains to investigate whether $\mathcal{L}_{V} \varphi=\mathcal{O}\left(\alpha^{\prime 2}\right)$. For this notice that under $G_{2}$ representations $d V$ decomposes as $d V=d V^{\mathbf{7}}+d V^{\mathbf{1 4}}+\mathcal{O}\left(\alpha^{\prime 2}\right)$ because $i_{V} d V=\mathcal{O}\left(\alpha^{\prime 2}\right)$. Then use (C.3) together with $\hat{\tilde{\nabla}} \varphi=\hat{\tilde{\nabla}} V=\mathcal{O}\left(\alpha^{\prime 2}\right)$ and $i_{V} d W=$ $\mathcal{O}\left(\alpha^{\prime 2}\right)$ to show that

$$
\hat{\tilde{\nabla}} d V^{7}=\mathcal{O}\left(\alpha^{\prime 2}\right) .
$$

As $d V^{\boldsymbol{7}}$ is a vector in $\mathcal{S}$ orthogonal to $V$, if it is not vanishing will generate an additional $\hat{\tilde{\nabla}}$-parallel spinor on $\mathcal{S}$ of the same chirality as $\eta_{+}$. As we have restricted the number of such spinors to one, we have to set $d V^{\mathbf{7}}=\mathcal{O}\left(\alpha^{2}\right)$. It has been shown in [25] that a $\hat{\tilde{\nabla}}$-parallel $\mathrm{k}$-form $\alpha$ is invariant under the action of a $\hat{\tilde{\nabla}}$-parallel vector $V$, iff the rotation $i_{V} W$ leaves the form invariant. As $i_{V} W=d V+\mathcal{O}\left(\alpha^{\prime 2}\right)$ and $d V$ takes values in $\mathfrak{g}_{2}$, we conclude that

$$
\mathcal{L}_{V} \varphi=\mathcal{O}\left(\alpha^{\prime 2}\right)
$$

and so $M^{7}$ admits a $G_{2}$ structure compatible with connection with skew-symmetric torsion given by the data $\left(d s_{(7)}^{2}, W_{(7)}\right)$. In such a case $W_{(7)}$ can be determined uniquely in terms of $\varphi$ and $d s_{(7)}^{2}$ provided a certain geometric constraint is satisfied [41].

It remains to explore (8.40) from the perspective of $M^{7}$. Let us decompose $h=V+h^{\perp}$, where $g\left(V, h^{\perp}\right)=0$. Then (8.40) can be written as

$$
\begin{aligned}
\ell^{-1} g(V, h)-\frac{1}{6}\left(W_{(7)}\right)_{i j k} \varphi^{i j k} & =\lambda \ell+\mathcal{O}\left(\alpha^{\prime 2}\right), \\
2 d \Phi+h^{\perp}-\theta_{\varphi} & =\mathcal{O}\left(\alpha^{\prime 2}\right)
\end{aligned}
$$

where $\theta_{\varphi}$ is the Lee form of $\varphi$ on $M^{7}$. The former determines the singlet part of $W_{(7)}$ in terms of $V$ and $h$ while the latter imposes the dilatino KSE on $M^{7}$.

\section{Nearly supersymmetric horizons with additional parallel spinors}

\subsection{Nearly supersymmetric horizons with SU(3) holonomy}

\subsubsection{Symmetries of horizon section}

Suppose there are exactly two linearly independent spinors $\eta_{+}^{(1)}, \eta_{+}^{(2)}$ such that

$$
\hat{\tilde{\nabla}} \eta_{+}^{(a)}=\mathcal{O}\left(\alpha^{2}\right), \quad a=1,2,
$$


for which $\left(\mathcal{A} \eta_{+}^{(a)}\right)^{[0]} \neq 0,(a=1,2)$. It follows that the horizon section $\mathcal{S}^{[0]}$ admits a $\mathrm{SU}(3)$ structure at zeroth order in $\alpha^{\prime}$.

We set $\tau_{+}^{(a)}=\mathcal{A} \eta_{+}^{(a)}$ which are non-vanishing spinors that satisfy

$$
\hat{\tilde{\nabla}} \tau_{+}^{(a)}=\mathcal{O}\left(\alpha^{\prime 2}\right), \quad a=1,2 .
$$

Using these we define the 1-form and 2-form spinor bilinears $V$ and $\omega$ by

$$
V_{i}=\left\langle\eta_{+}^{(1)}, \Gamma_{i} \tau_{+}^{(1)}\right\rangle, \quad \omega_{i j}=\left\langle\eta_{+}^{(1)}, \Gamma_{i j} \eta_{+}^{(2)}\right\rangle,
$$

and also let

$$
\tilde{V}=i_{V} \omega
$$

Observe that

$$
\hat{\tilde{\nabla}} V=\mathcal{O}\left(\alpha^{\prime 2}\right), \quad \hat{\tilde{\nabla}} \omega=\mathcal{O}\left(\alpha^{2}\right), \quad \hat{\tilde{\nabla}} \tilde{V}=\mathcal{O}\left(\alpha^{\prime 2}\right) .
$$

We also define $\tilde{h}$ by

$$
\tilde{h}=i_{h} \omega
$$

which satisfies

$$
\hat{\tilde{\nabla}} \tilde{h}=\mathcal{O}\left(\alpha^{\prime}\right) .
$$

The main task below is to show that both $V$ and $\tilde{V}$ leave invariant all the fields on $\mathcal{S}$, and that they generate a $\mathbb{R} \oplus \mathbb{R}$ lie algebra.

As $V$ and $\tilde{V}$ are $\hat{\tilde{\nabla}}$-parallel, they are Killing. Next consider the invariance of $W$. The spinors $V^{j} \Gamma_{j} \mathcal{A} \eta_{+}^{(a)}, h^{j} \Gamma_{j} \mathcal{A} \eta_{+}^{(a)}$ and $\tilde{h}^{j} \Gamma_{j} \mathcal{A} \eta_{+}^{(a)}$ are all parallel with respect to $\hat{\tilde{\nabla}}$ to zeroth order in $\alpha^{\prime}$. In order for (9.1) to have exactly two solutions, we must have

$$
V=c h+\tilde{c} \tilde{h}+\mathcal{O}\left(\alpha^{\prime}\right)
$$

for some constants $c, \tilde{c}$. Thus

$$
\mathcal{L}_{V} W=c i_{h} d W+\tilde{c} i_{\tilde{h}} d W+\mathcal{O}\left(\alpha^{\prime 2}\right) .
$$

To continue, since the two spinors $\eta_{+}^{(1)}$ and $\eta_{+}^{(2)}$ must satisfy (8.7), it follows that, at zeroth order in $\alpha^{\prime}, \tilde{F}$ and $d h$ are $(1,1)$ traceless with respect to the almost complex structure obtained from $\omega$. This, together with the conditions $i_{h} d h=\mathcal{O}\left(\alpha^{\prime}\right)$ and $i_{h} \tilde{F}=\mathcal{O}\left(\alpha^{\prime}\right)$, which follow from the global analysis of the Laplacian of $h^{2}$, implies that

$$
i_{\tilde{h}} d h=\mathcal{O}\left(\alpha^{\prime}\right), \quad i_{\tilde{h}} \tilde{F}=\mathcal{O}\left(\alpha^{\prime}\right),
$$

and hence

$$
i_{V} d h=\mathcal{O}\left(\alpha^{\prime}\right), \quad i_{V} \tilde{F}=\mathcal{O}\left(\alpha^{\prime}\right) .
$$

It is also useful to consider the spinors $\eta_{+}^{(a)}$ and $\tilde{h}_{\ell} \Gamma^{\ell} \eta_{+}^{(a)}$. The integrability conditions of

$$
\hat{\tilde{\nabla}} \eta_{+}^{(a)}=\mathcal{O}\left(\alpha^{\prime 2}\right), \quad \hat{\tilde{\nabla}}\left(\tilde{h}_{\ell} \Gamma^{\ell} \eta_{+}^{(a)}\right)=\mathcal{O}\left(\alpha^{\prime}\right),
$$


are

$$
\hat{\tilde{R}}_{i j, p q} \Gamma^{p q} \eta_{+}^{(a)}=\mathcal{O}\left(\alpha^{\prime 2}\right), \quad \hat{\tilde{R}}_{i j, p q} \Gamma^{p q}\left(\tilde{h}_{\ell} \Gamma^{\ell} \eta_{+}^{(a)}\right)=\mathcal{O}\left(\alpha^{\prime}\right),
$$

which imply

$$
\tilde{h}^{p} \tilde{\tilde{R}}_{p q, i j}=\mathcal{O}\left(\alpha^{\prime}\right) .
$$

It follows that $i_{h} d W=\mathcal{O}\left(\alpha^{\prime 2}\right)$ and $i_{\tilde{h}} d W=\mathcal{O}\left(\alpha^{\prime 2}\right)$, as a consequence of the Bianchi identity, and therefore $i_{V} d W=\mathcal{O}\left(\alpha^{\prime 2}\right)$. Thus we have shown that

$$
\mathcal{L}_{V} W=\mathcal{O}\left(\alpha^{\prime 2}\right) .
$$

This proves the invariance of $W$.

Next we consider $\mathcal{L}_{V} \Phi$. It follows from (9.7) that

$$
i_{\tilde{h}} d \tilde{h}=\mathcal{O}\left(\alpha^{\prime}\right)
$$

and also

$$
\mathcal{L}_{\tilde{h}} W=\mathcal{O}\left(\alpha^{\prime}\right) .
$$

Since $\tilde{h}$ is an isometry of $\mathcal{S}$ to zeroth order in $\alpha^{\prime}$, we also have

$$
\mathcal{L}_{\tilde{h}} \tilde{R}_{i j, p q}=\mathcal{O}\left(\alpha^{\prime}\right)
$$

On taking the Lie derivative of the trace of (4.16) with respect to $\tilde{h}$, we find

$$
\mathcal{L}_{\tilde{h}}\left(\tilde{\nabla}_{i} \tilde{\nabla}^{i} \Phi\right)=\mathcal{O}\left(\alpha^{\prime}\right)
$$

which is equivalent, if $g=\mathcal{L}_{\tilde{h}} \Phi$, to

$$
\tilde{\nabla}_{i} \tilde{\nabla}^{i} g=\mathcal{O}\left(\alpha^{\prime}\right)
$$

On integrating the zeroth order of (9.20), we find

$$
\int_{\mathcal{S}^{[0]}} \tilde{\nabla}_{i} g^{[0]} \tilde{\nabla}^{i} g^{[0]}=0,
$$

so $g^{[0]}=\gamma$, for constant $\gamma$. Thus

$$
\mathcal{L}_{\tilde{h}} \Phi=\gamma+\mathcal{O}\left(\alpha^{\prime}\right)
$$

Since $\Phi$ must attain a global maximum on $\mathcal{S}$, at this point $\mathcal{L}_{\tilde{h}} \Phi=0$ to all orders in $\alpha^{\prime}$. This fixes the constant $\gamma=0$, and so

$$
\mathcal{L}_{\tilde{h}} \Phi=\mathcal{O}\left(\alpha^{\prime}\right),
$$

which implies

$$
\mathcal{L}_{V} \Phi=\mathcal{O}\left(\alpha^{\prime}\right) .
$$

As $V=c h+\tilde{c} \tilde{h}+\mathcal{O}\left(\alpha^{\prime}\right)$, it follows that

$$
\mathcal{L}_{V} d h=c \mathcal{L}_{h} d h+\tilde{c} \mathcal{L}_{\tilde{h}} d h+\mathcal{O}\left(\alpha^{\prime}\right)=\mathcal{O}\left(\alpha^{\prime}\right) .
$$


Since $V$ is an isometry of $\mathcal{S}$ to first order in $\alpha^{\prime}$, we have

$$
\mathcal{L}_{V} \tilde{R}_{i j, p q}=\mathcal{O}\left(\alpha^{\prime 2}\right) .
$$

Also we have

$$
\left(\mathcal{L}_{V} \tilde{F}\right)_{i j}{ }_{b}{ }_{b} \tilde{F}^{i j b}{ }_{a}=\mathcal{O}\left(\alpha^{\prime}\right),
$$

which follows from

$$
\mathcal{L}_{V} \tilde{F}=c\left[\tilde{F}, i_{h} \mathcal{B}\right]+\tilde{c}\left[\tilde{F}, i_{\tilde{h}} \mathcal{B}\right]+\mathcal{O}\left(\alpha^{\prime}\right) .
$$

Using the conditions (9.24), (9.25), (9.26) and (9.27), we follow the analysis for the $G_{2}$ case of the previous section undertaken from the equation (8.22) to (8.29), and conclude that

$$
\mathcal{L}_{V} \Phi=\mathcal{O}\left(\alpha^{\prime 2}\right)
$$

which proves the invariance of the dilaton $\Phi$.

Next we consider $\mathcal{L}_{V} h$. Equations (8.30) and (8.31), which have been established in the previous section, hold here as well after using in the addition that

$$
\mathcal{L}_{\tilde{h}}\left(\tilde{F}_{i \ell}^{a b} \tilde{F}_{j}^{\ell} a b\right)=\mathcal{O}\left(\alpha^{\prime}\right) .
$$

Then it follows that

$$
\hat{\tilde{\nabla}}_{i}\left(\mathcal{L}_{V} h\right)_{j}=\mathcal{O}\left(\alpha^{\prime 2}\right) .
$$

Furthermore we notice that

$$
\mathcal{L}_{\tilde{h}} h=\mathcal{O}\left(\alpha^{\prime}\right) .
$$

As $V=c h+\tilde{c} \tilde{h}+\mathcal{O}\left(\alpha^{\prime}\right)$, it is convenient to write

$$
\mathcal{L}_{V} h=\alpha^{\prime} \Psi+\mathcal{O}\left(\alpha^{\prime 2}\right),
$$

where

$$
\hat{\tilde{\nabla}} \Psi=\mathcal{O}\left(\alpha^{\prime}\right) .
$$

Then it follows that the spinors $\Psi_{j} \Gamma^{j} \mathcal{A} \eta_{+}, h_{j} \Gamma^{j} \mathcal{A} \eta_{+}$and $\tilde{h}_{j} \Gamma^{j} \mathcal{A} \eta_{+}$are all parallel with respect to $\hat{\tilde{\nabla}}$ at zeroth order in $\alpha^{\prime}$. In order for (9.1) to admit exactly two solutions, we must have

$$
\Psi=b h+\tilde{b} \tilde{h}+\mathcal{O}\left(\alpha^{\prime}\right),
$$

for constants $b$ and $\tilde{b}$. Then using $i_{h} \mathcal{L}_{V} h=\mathcal{O}\left(\alpha^{\prime 2}\right)$, which has been computed in (8.37), and $h^{2}=$ const. $+\mathcal{O}\left(\alpha^{\prime 2}\right)$, it follows that $b=\mathcal{O}\left(\alpha^{\prime}\right)$ and therefore

$$
\mathcal{L}_{V} h=\alpha^{\prime} \tilde{b} \tilde{h}+\mathcal{O}\left(\alpha^{\prime 2}\right)
$$

Next we consider the symmetries generated by $\tilde{V}$. Since $V=c h+\tilde{c} \tilde{h}+\mathcal{O}\left(\alpha^{\prime}\right)$, then we have

$$
\tilde{V}=c \tilde{h}-\tilde{c} h+\mathcal{O}\left(\alpha^{\prime}\right)
$$


Since $V$ and $\omega$ are both parallel with respect to $\hat{\tilde{\nabla}}$ to first order in $\alpha^{\prime}$, we also have

$$
\hat{\tilde{\nabla}} \tilde{V}=\mathcal{O}\left(\alpha^{\prime 2}\right) .
$$

Then the analysis undertaken for $V$ holds as well for $\tilde{V}$, because the only properties of $V$ used through the analysis are that $V$, at zeroth order in $\alpha^{\prime}$, is a linear combination of $h$ and $\tilde{h}$ with constant coefficients, and $V$ is parallel with respect to $\hat{\tilde{\nabla}}$ to first order in $\alpha^{\prime}$. Thus we argue in a similar way that

$$
\mathcal{L}_{\tilde{V}} W=\mathcal{O}\left(\alpha^{\prime 2}\right), \quad \mathcal{L}_{\tilde{V}} \Phi=\mathcal{O}\left(\alpha^{\prime 2}\right), \quad \mathcal{L}_{\tilde{V}} h=\alpha^{\prime} \tilde{q} \tilde{h}+\mathcal{O}\left(\alpha^{\prime 2}\right)
$$

for a constant $\tilde{q}$.

Finally, the $V$ and $\tilde{V}$ commute up to $\mathcal{O}\left(\alpha^{2}\right)$. To see this observe that since $i_{V} \tilde{V}=0$ and $i_{V} W=d V+\mathcal{O}\left(\alpha^{\prime 2}\right)$, we have that

$$
\mathcal{L}_{\tilde{V}} V=i_{\tilde{V}} i_{V} W+\mathcal{O}\left(\alpha^{\prime 2}\right)
$$

Using (C.3) adapted to $\mathcal{S}$ as well as $i_{V} d W=i_{\tilde{V}} d W=\mathcal{O}\left(\alpha^{\prime 2}\right)$, we conclude that

$$
\hat{\tilde{\nabla}} i_{\tilde{V}} i_{V} W=\mathcal{O}\left(\alpha^{\prime 2}\right)
$$

Therefore the vector $i_{\tilde{V}} i_{V} W$ is $\hat{\tilde{\nabla}}$-parallel and moreover is orthogonal to both $V$ and $\tilde{V}$. So if it is non-zero, it will generate additional $\hat{\tilde{\nabla}}$-parallel $\eta_{+}$spinors on $\mathcal{S}$. As we have restricted those to be strictly two, we conclude that $i_{\tilde{V}} i_{V} W$ vanishes and so

$$
[V, \tilde{V}]=\mathcal{O}\left(\alpha^{\prime 2}\right)
$$

In particular as $i_{V} \tilde{V}=0$, we have that

$$
i_{V} d \tilde{V}=i_{\tilde{V}} d V=\mathcal{O}\left(\alpha^{\prime 2}\right) .
$$

This concludes the examination of the symmetries of $\mathcal{S}$.

\subsubsection{Geometry}

It is clear from the examination of the symmetries of the fields on $\mathcal{S}$ and in particular (9.5) and (9.43) that we can set

$$
\begin{aligned}
d \tilde{s}^{2} & =\ell^{-2} V \otimes V+\ell^{-2} \tilde{V} \otimes \tilde{V}+d s_{(6)}^{2}+\mathcal{O}\left(\alpha^{\prime 2}\right), \\
W & =\ell^{-2} V \wedge d V+\ell^{-2} \tilde{V} \wedge d \tilde{V}+W_{(6)}+\mathcal{O}\left(\alpha^{\prime 2}\right),
\end{aligned}
$$

where $V^{2}=\tilde{V}^{2}=\ell^{2}+\mathcal{O}\left(\alpha^{\prime 2}\right)$ and $\ell$ is constant, $d s_{(6)}^{2}$ is the metric in the orthogonal complement of $V$ and $\tilde{V}$ and $i_{V} W_{(6)}=i_{\tilde{V}} W_{(6)}=\mathcal{O}\left(\alpha^{\prime 2}\right)$.

From construction $\mathcal{S}$ admits an $\mathrm{SU}(3)$ structure. We shall now investigate whether this (locally) descends on the space of orbits $M^{6}$ of $V$ and $\tilde{V}$. First the data $\left(d s_{(6)}^{2}, W_{(6)}\right)$ define a Riemannian geometry on $M^{6}$ with skew-symmetric torsion. In particular for the torsion this follows from $i_{V} W_{(6)}=i_{\tilde{V}} W_{(6)}=\mathcal{O}\left(\alpha^{\prime 2}\right)$ and $\mathcal{L}_{V} W_{(6)}=\mathcal{L}_{\tilde{V}} W_{(6)}=\mathcal{O}\left(\alpha^{2}\right)$. 
Next consider the reduction of the (almost) Hermitian form $\omega$. Choosing without loss of generality $V$ and $\tilde{V}$ orthogonal, one can write

$$
\omega=\ell^{-2} V \wedge \tilde{V}+\omega_{(6)}+\mathcal{O}\left(\alpha^{\prime 2}\right)
$$

where $i_{V} \omega_{(6)}=i_{\tilde{V}} \omega_{(6)}=\mathcal{O}\left(\alpha^{\prime 2}\right)$. For $\omega_{(6)}$ to descend to a Hermitian structure on $M^{6}$, it must be invariant under the action of both $V$ and $\tilde{V}$. Observe that $\hat{\tilde{\nabla}} \omega_{(6)}=\mathcal{O}\left(\alpha^{\prime 2}\right)$ and also $\hat{\tilde{\nabla}} V=\hat{\tilde{\nabla}} \tilde{V}=\mathcal{O}\left(\alpha^{\prime 2}\right)$. Thus $\omega_{(6)}$ is invariant iff the rotations $i_{V} W=d V+\mathcal{O}\left(\alpha^{\prime 2}\right)$ and $i_{\tilde{V}} W=d \tilde{V}+\mathcal{O}\left(\alpha^{\prime 2}\right)$ leave $\omega_{(6)}$ invariant [25]. In turn this implies that the $(2,0)$ and $(0,2)$ parts of the rotations which we denote with $[d V]^{2,0}$ and $[d \tilde{V}]^{2,0}$, respectively, must vanish. Using (C.3), $\hat{\tilde{\nabla}} \omega_{(6)}=\mathcal{O}\left(\alpha^{2}\right)$ and $i_{V} d W=i_{\tilde{V}} d W=\mathcal{O}\left(\alpha^{\prime 2}\right)$, we find that

$$
\hat{\tilde{\nabla}}\left[i_{V} W\right]^{2,0}=\hat{\tilde{\nabla}}\left[i_{\tilde{V}} W\right]^{2,0}=\mathcal{O}\left(\alpha^{\prime 2}\right) .
$$

As $\mathcal{S}$ has an $\mathrm{SU}(3)$ structure compatible with $\hat{\tilde{\nabla}}$, contracting with the (3,0)-form both $\left[i_{\tilde{V}} W\right]^{2,0}$ and $\left[i_{\tilde{V}} W\right]^{2,0}$ give rise to vector fields in $\mathcal{S}$ orthogonal to both $V$ and $\tilde{V}$ which are $\hat{\tilde{\nabla}}$-parallel. Thus the requirement of strictly two $\eta_{+} \hat{\tilde{\nabla}}$-parallel spinors leads to setting $\left[i_{\tilde{V}} W\right]^{2,0}=\left[i_{\tilde{V}} W\right]^{2,0}=\mathcal{O}\left(\alpha^{\prime 2}\right)$ which in turn implies that

$$
\mathcal{L}_{V} \omega_{(6)}=\mathcal{L}_{\tilde{V}} \omega_{(6)}=\mathcal{O}\left(\alpha^{\prime 2}\right) .
$$

Thus $M^{6}$ admits an almost Hermitian structure compatible with a connection $\hat{\tilde{\nabla}}^{(6)}$ with skew-symmetric torsion $W_{(6)}$. It is well known that in this case $W_{(6)}$ is determined in terms of the almost complex structure on $M^{6}$ and the metric, see eg [42].

To find whether $M^{6}$ inherits a $\mathrm{SU}(3)$ structure as well, let investigate whether the $(3,0)$ fundamental $\mathrm{SU}(3)$ form $\chi$ of $\mathcal{S}$ descends on $M^{6}$. It can always be arranged such that $i_{V} \chi=i_{\tilde{V}} \chi=0$. So it remains to see whether $\chi$ is invariant under the action of $V$ and $\tilde{V}$. For this a similar argument to that explained above for $\omega_{(6)}$ leads to the assertion that $\chi$ is invariant iff the $\omega$-traces $i_{\tilde{V}} W \cdot \omega$ and $i_{\tilde{V}} W \cdot \omega$ of $i_{\tilde{V}} W$ and $i_{\tilde{V}} W$, respectively, vanish. Furthermore, an application of (C.3) implies that both $i_{\tilde{V}} W \cdot \omega$ and $i_{\tilde{V}} W \cdot \omega$ are constant but not necessarily zero. Thus $M^{6}$ has generically a U(3) structure instead of an $\mathrm{SU}(3)$ one.

It remains to investigate the rest of the content of the conditions $\hat{\tilde{\nabla}} \tau_{+}^{(a)}=\mathcal{O}\left(\alpha^{2}\right)$. First consider the $(3,0)$ part of $W_{(6)}$ denoted by $W_{(6)}^{3,0}$. An application of (C.3) using that $d W$ is a $(2,2)$ form yields that

$$
\hat{\tilde{\nabla}} W_{(6)}^{3,0}=\mathcal{O}\left(\alpha^{\prime 2}\right)
$$

Thus $W_{(6)}^{3,0}$ is another globally defined $\hat{\tilde{\nabla}}$-parallel $(3,0)$-form on $\mathcal{S}$ and so it can either be set to zero or be identified with $\chi$. In the former case, the complex structure on $M^{6}$ is integrable and so $M^{6}$ is a KT manifold [43].

Writing $h=\lambda_{1} V+\lambda_{2} \tilde{V}+h^{\perp}$, where $h^{\perp}$ is orthogonal to both $V$ and $\tilde{V}$ and $\lambda_{1}$ and $\lambda_{2}$ are constants, we find using (C.3) that

$$
\hat{\tilde{\nabla}}\left(2 d \Phi+h^{\perp}-\theta_{\omega_{(6)}}\right)=\mathcal{O}\left(\alpha^{\prime 2}\right) .
$$


Now if $2 d \Phi+h^{\perp}-\theta_{\omega_{(6)}}$ in non-vanishing and since it is orthogonal to $V$ and $\tilde{V}$ will give rise to more than two $\eta_{+} \hat{\tilde{\nabla}}$-parallel spinors on $\mathcal{S}$. Since we have assumed that there are just two, we set

$$
2 d \Phi+h^{\perp}-\theta_{\omega_{(6)}}=\mathcal{O}\left(\alpha^{\prime 2}\right) .
$$

This concludes the investigation of the geometry.

\subsection{Nearly supersymmetric horizons with SU(2) holonomy}

\subsubsection{Assumptions and definitions}

It is known that if one requires the existence of an additional $\hat{\tilde{\nabla}}$-parallel spinor $\eta_{+}$to those of the $\mathrm{SU}(3)$ backgrounds on $\mathcal{S}$, then the isotropy algebra of the all the five spinors reduces to $\mathfrak{s u}(2)$. As a result, $\mathcal{S}$ admits $8 \hat{\tilde{\nabla}}$-parallel spinors and the holonomy group reduces to a subgroup $\mathrm{SU}(2)$. To describe the geometry of backgrounds with exactly 8 such spinors, we consider four linearly independent spinors $\eta_{+}^{(a)}$, and impose the condition

$$
\hat{\tilde{\nabla}} \eta_{+}^{(a)}=\mathcal{O}\left(\alpha^{2}\right), \quad a=0,1,2,3,
$$

for which $\left(\mathcal{A} \eta_{+}^{(a)}\right)^{[0]} \neq 0,(a=0,1,2,3)$. It follows that the horizon section $\mathcal{S}^{[0]}$ admits a SU(2) structure at zeroth order in $\alpha^{\prime}$. We continue by setting $\tau_{+}^{(a)}=\mathcal{A} \eta_{+}^{(a)}$. These are non-vanishing and satisfy

$$
\hat{\tilde{\nabla}} \tau_{+}^{(a)}=\mathcal{O}\left(\alpha^{2}\right), \quad a=0,1,2,3 .
$$

Furthermore, we also define 1-form and 2-form spinor bilinears $V^{(a)}$ and $\omega_{r}$, respectively, by

$$
V_{i} \equiv V_{i}^{(0)}=\left\langle\eta_{+}^{(0)}, \Gamma_{i} \tau_{+}^{(0)}\right\rangle, \quad\left(\omega_{r}\right)_{i j}=\left\langle\eta_{+}^{(0)}, \Gamma_{i j} \eta_{+}^{(r)}\right\rangle, \quad r=1,2,3,
$$

and also let

$$
\tilde{V}_{r}=i_{V} \omega_{r} .
$$

In fact $\omega_{r}$ together with the metric and $W$ define an almost HKT structure [43] on $\mathcal{S}$ as

$$
\hat{\tilde{\nabla}} V=\mathcal{O}\left(\alpha^{\prime 2}\right), \quad \hat{\tilde{\nabla}} \omega_{r}=\mathcal{O}\left(\alpha^{2}\right), \quad \hat{\tilde{\nabla}} \tilde{V}_{r}=\mathcal{O}\left(\alpha^{\prime 2}\right),
$$

and the almost complex structures associated to $\omega_{r}$ satisfy the algebra of unit quaternions. These follow from (8.8) and the $\mathfrak{s u}(2)$ isotropy of the parallel spinors.

\subsubsection{Symmetries of the horizon section}

It is clear from (9.55) that $V^{(a)}, V^{(r)}=V_{r}$, generate isometries on $\mathcal{S}$ and that

$$
i_{a} W=d V^{(a)}+\mathcal{O}\left(\alpha^{\prime 2}\right)
$$

where $i_{a}$ denotes inner-derivation with respect to $V^{(a)}$. Without loss of generality we choose $g\left(V^{(a)}, V^{(b)}\right)=\ell^{2} \delta^{a b}+\mathcal{O}\left(\alpha^{\prime 2}\right)$ for $\ell$ constant. An investigation similar to the one explained in section 9.1 .1 reveals that

$$
\begin{aligned}
\mathcal{L}_{a} \Phi & =\mathcal{O}\left(\alpha^{\prime 2}\right), \quad \mathcal{L}_{a} W=\mathcal{O}\left(\alpha^{\prime 2}\right), \quad \mathcal{L}_{a} h=\mathcal{O}\left(\alpha^{\prime}\right), \quad i_{a} d h=\mathcal{O}\left(\alpha^{\prime}\right), \\
i_{a} F & =\mathcal{O}\left(\alpha^{\prime}\right) .
\end{aligned}
$$


Next let us consider the commutator $\left[V^{(a)}, V^{(b)}\right]=i_{a} i_{b} W$. An application of (C.3) together with the conditions above reveal that

$$
\hat{\tilde{\nabla}}\left[V^{(a)}, V^{(b)}\right]=\mathcal{O}\left(\alpha^{\prime 2}\right) .
$$

Thus the commutator is either linear dependent on $V^{(a)}$ or it will lead to further reduction of the holonomy of $\hat{\tilde{\nabla}}$ to $\{1\}$. In the latter case, the horizon section $\mathcal{S}$ will admit more than four $\eta_{+} \hat{\tilde{\nabla}}$-parallel spinors violating our assumptions. Thus, we conclude that

$$
\left[V^{(a)}, V^{(b)}\right]=f^{a b}{ }_{c} V^{(c)}+\mathcal{O}\left(\alpha^{2}\right),
$$

for some constants $f$ with $\ell^{2} f^{a b}{ }_{c}=i_{a} i_{b} i_{c} W+\mathcal{O}\left(\alpha^{2}\right)$. As $f$ is skew-symmetric, the Lie algebra spanned by $V^{(a)}$ is a metric (compact) Lie algebra. As it has dimension 4 , it is either isomorphic to $\oplus^{4} \mathfrak{u}(1)$ or to $\mathfrak{u}(1) \oplus \mathfrak{s u}(2)$.

Therefore the horizon section $\mathcal{S}$ can be viewed locally as a fibration with fibre either $\times{ }^{4} \mathrm{U}(1)$ or $\mathrm{U}(1) \times \mathrm{SU}(2)$ over the space of orbits $M^{4}$ of $V^{(a)}$. We shall determine the geometry of $\mathcal{S}$ by specifying the geometry of $M^{4}$.

\subsubsection{Geometry}

To simplify the analysis, we choose up to an $\mathfrak{s o}(4)$ rotation $V$ to be along a $\mathfrak{u}(1)$ direction in either $\oplus^{4} \mathfrak{u}(1)$ or $\mathfrak{u}(1) \oplus \mathfrak{s u}(2)$. This in particular implies that $i_{0} i_{r} W=\mathcal{O}\left(\alpha^{\prime 2}\right)$. Then the metric and torsion of $\mathcal{S}$ can be written as

$$
\begin{aligned}
d \tilde{s}^{2} & =\ell^{-2} \delta_{a b} V^{(a)} \otimes V^{(b)}+d \tilde{s}_{(4)}^{2}+\mathcal{O}\left(\alpha^{\prime 2}\right), \\
W & =\ell^{-2} V \wedge d V+\operatorname{CS}\left(V_{r}\right)+W_{(4)}+\mathcal{O}\left(\alpha^{\prime 2}\right),
\end{aligned}
$$

where $V^{(a)}$ is viewed as a principal bundle connection and $\operatorname{CS}\left(V_{r}\right)$ is the Chern-Simons form which for the $\oplus^{4} \mathfrak{u}(1)$ case is

$$
\mathrm{CS}\left(V_{r}\right)=\ell^{-2} \sum_{r} V_{r} \wedge d V_{r}
$$

The data $\left(d s_{(4)}^{2}, W_{(4)}\right)$ define a geometry on $M^{4}$ with skew-symmetric torsion.

First, let us investigate the reduction of the almost HKT structure of $\mathcal{S}$ on $M^{4}$. For this observe that

$$
\omega_{r}=\ell^{-2} V \wedge V_{r}+\frac{\ell^{-2}}{2} \epsilon_{r}^{s t} V_{s} \wedge V_{t}+\omega_{r}^{(4)}+\mathcal{O}\left(\alpha^{\prime 2}\right),
$$

where $i_{a} \omega_{r}^{(4)}=\mathcal{O}\left(\alpha^{\prime 2}\right)$. Next consider $\mathcal{L}_{a} \omega_{r}^{(4)}$. As both $V^{(a)}$ and $\omega_{r}^{(4)}$ are $\hat{\tilde{\nabla}}$-parallel, $\mathcal{L}_{a} \omega_{r}^{(4)}$ is specified by the properties of the rotation $i_{a} W$. In particular if $i_{a} W$ is invariant under $\omega_{r}^{(4)}$, the Lie derivative vanishes.

Next let us investigate the two cases $\oplus^{4} \mathfrak{u}(1)$ and $\mathfrak{u}(1) \oplus \mathfrak{s u}(2)$ separately. In the abelian case, as $i_{a} i_{b} W=\mathcal{O}\left(\alpha^{2}\right), i_{a} W$ is a 2 -form on $M^{4}$. Furthermore $\mathcal{L}_{a} \omega_{r}^{(4)}$ vanishes iff the self-dual part, $i_{a} W^{\text {sd }}$, of $i_{a} W$ is zero. However in general this may not be the case. An application of (C.3) implies that

$$
\hat{\tilde{\nabla}} i_{a} W^{\text {sd }}=\mathcal{O}\left(\alpha^{\prime 2}\right)
$$


and so there exist some constants $u$ such that

$$
i_{a} W^{\mathrm{sd}}=u_{a}{ }^{r} \omega_{r}^{(4)}+\mathcal{O}\left(\alpha^{\prime 2}\right),
$$

otherwise the holonomy of $\hat{\tilde{\nabla}}$ will be reduced further and it will admit more than four $\eta_{+}$ parallel spinors. Then

$$
\mathcal{L}_{a} \omega_{r}^{(4)}=2 u_{a}{ }^{s} \epsilon_{s r}{ }^{t} \omega_{t}^{(4)}+\mathcal{O}\left(\alpha^{\prime 2}\right)
$$

The identity $\left[\mathcal{L}_{a}, \mathcal{L}_{b}\right]=\mathcal{L}_{\left[V^{(a)}, V^{(b)}\right]}$ gives

$$
\left(u_{a}^{r} u_{b}^{s}-u_{b}^{r} u_{a}^{s}\right)=\mathcal{O}\left(\alpha^{\prime 2}\right) .
$$

The covariant constancy condition on $M^{4}$ now reads

$$
\hat{\tilde{\nabla}}^{(4)} \omega_{r}^{(4)}=2 \ell^{-2} V^{(a)} u_{a}^{s} \epsilon_{s r}{ }^{t} \omega_{t}^{(4)}+\mathcal{O}\left(\alpha^{\prime 2}\right),
$$

where now $V^{(a)}$ should be thought as the pull back of the principal bundle connection $V^{(a)}$ with a local section. It is clear that the relevant connection that determines the geometry of $M^{4}$ is $Z^{s}=V^{(a)} u_{a}^{s}$.

If $u_{a}^{r}=0, M^{4}$ is a HKT manifold. It is easy to see this as $\omega_{r}$ are covariantly constant with respect to a connection with skew-symmetric torsion and all three almost complex structures are integrable. The latter follows because of dimensional reasons. Otherwise one of the 3 -vectors $u_{a}$ must be non-zero. Without loss of generality take $u_{0} \neq 0$. In such a case the above equation can be solved as $\left(u_{a}^{r}\right)=\left(u_{0}^{r}, u_{0}^{r} v_{s}\right)$, where $v_{s}=\left|u_{0}\right|^{-2} \sum_{r} u_{s}^{r} u_{0}^{r}$.

Using these data, the covariant constancy condition of $\omega_{r}^{(4)}$ on $M^{4}$ can be written as

$$
\hat{\tilde{\nabla}}^{(4)} \omega_{r}^{(4)}=2 \ell^{-2}\left(V^{0}+V^{p} v_{p}\right) u_{0}^{s} \epsilon_{s r}{ }^{t} \omega_{t}^{(4)}+\mathcal{O}\left(\alpha^{\prime 2}\right)
$$

It is clear from this that $M^{4}$ is a $\mathrm{KT}$ manifold with respect to the Hermitian form $\left|u_{0}\right|^{-1} u_{0}^{r} \omega_{r}$. In fact $M^{4}$ is an (almost) ${ }^{4}$ QKT manifold [44] for which the holonomy of the $\operatorname{Sp}(1)$ connection has been reduced to $\mathrm{U}(1)$.

Next let us turn to examine the non-abelian $\mathfrak{u}(1) \oplus \mathfrak{s u}(2)$ case. It is easy to see that

$$
\left(u_{a}^{r} u_{b}^{s}-u_{b}^{r} u_{a}^{s}\right)=\frac{1}{2} f^{a b}{ }_{c} u_{c}^{t} \epsilon_{t r}{ }^{s}+\mathcal{O}\left(\alpha^{\prime 2}\right) .
$$

If the 3 -vector $u_{0} \neq 0$, then all the rest of the components of $u$ vanish. In such a case, $M^{4}$ is an KT manifold. This class of solutions includes the WZW type of solution $\operatorname{AdS}_{3} \times S^{3} \times M^{4}$ where $M^{4}=S^{1} \times S^{3}$ with the bi-invariant metric and constant dilaton. Such a horizon is not supersymmetric but it is nearly supersymmetric.

It remains to consider the case $u_{0}=0$. One can then show that $\operatorname{det} u \neq 0$ and so $\left(u_{s}^{r}\right)$ is invertible. Thus $Z^{s}=V^{(a)} u_{a}^{s}$ takes values in the $\mathfrak{s p}(1)$ Lie algebra. $M^{4}$ is a QKT manifold, see also [28].

To conclude we remark that in all HKT and KT cases, there is an analogue of the condition (9.49) for every Hermitian form $\omega_{r}$ that determines these structures. If the

\footnotetext{
${ }^{4}$ In the definition of QKT structure in [44] an additional integrability condition was considered.
} 
associated $2 d \Phi+h^{\perp}-\theta_{r}$ forms do not vanish, then the holonomy of the connection with torsion reduces to $\{1\}$ and the number of parallel spinors enhance to 16 . The solutions are the group manifolds. The solution $\mathrm{AdS}_{3} \times S^{3} \times S^{3} \times S^{1}$ mentioned above belongs to the class where the holonomy of the connection with torsion is $\{1\}$.

There is an analogue of this in the QKT case but in such a case the condition from the perspective of $M^{4}$ twists with $\mathfrak{s p}(1)$. If $2 d \Phi+h^{\perp}-\theta_{r}$ do not vanish, again the holonomy of the connection with torsion on $\mathcal{S}$ reduces to $\{1\}$. However now some of the data like the Hermitian forms are not (bi-)invariant under the action of the group. It would be of interest to explore his further to see whether there are actual solutions.

We conclude the examination of the geometry of nearly supersymmetric backgrounds in the $G_{2}, \mathrm{SU}(3)$ and $\mathrm{SU}(2)$ cases by pointing out that they exhibit an $\mathfrak{s l}(2, \mathbb{R})$ up to order $\mathcal{O}\left(\alpha^{\prime}\right)$ but not up to order $\mathcal{O}\left(\alpha^{\prime 2}\right)$. For the latter, $h$ must be a symmetry of the theory up to the same order and so it can be identified with $V$. The description of the geometry of this special class of nearly supersymmetric backgrounds is very similar to the one we have given above. The only difference is that now we can identify $h$ with $V$.

\section{Conclusions}

We have investigated the supersymmetric near-horizon geometry of heterotic black holes up to and including two loops in sigma model perturbation theory. Using a combination of local and global techniques, together with the bosonic field equations and Bianchi identities, we have proven that the conditions obtained from the KSEs are equivalent to a pair of gravitino equations (4.26) and a pair of algebraic conditions, related to the dilatino KSE, (4.27), which are required to hold at zeroth and first order in $\alpha^{\prime}$. In particular, we have shown that the KSE related to the gaugino is implied by the other KSEs and field equations.

In all cases, we have also shown that there are no regular $\mathrm{AdS}_{2}$ solutions with compact without boundary internal space by demonstrating that $\Delta=\mathcal{O}\left(\alpha^{\prime 2}\right)$. This is not in contradiction with the fact that one can locally write $\mathrm{AdS}_{3}$ as a warped product over $\mathrm{AdS}_{2}$ [45], see also appendix E. This is because our assumptions on the internal space of $\mathrm{AdS}_{2}$ are violated in such a case.

Furthermore, we have demonstrated that horizons that admit a non-vanishing $\eta_{-}$ Killing spinor up to order $\mathcal{O}\left(\alpha^{\prime 2}\right)$, which does not vanish at zeroth order in $\alpha^{\prime}$, exhibit supersymmetry enhancement via the same mechanism as described in [27], and so preserve 2, 4, 6 and 8 supersymmetries. We have described the geometry of such horizons in all cases and this is similar to that presented in [27] for the horizons with $d H=0$.

We have also considered in some detail the global properties of our solutions. The analysis of the global properties of $h^{2}$ proceeds in much the same way as in the heterotic theory with $d H=0$. However in the presence of anomaly, the consequences of the global restrictions on the geometry of the horizons are somewhat weaker. For example, it is only possible to prove that $h$ is an isometry of the horizon section to zeroth order in $\alpha^{\prime}$. So one cannot establish a direct algebraic relation between $\eta_{+}$and $\eta_{-}$spinors to order $\mathcal{O}\left(\alpha^{\prime 2}\right)$, and 
therefore it is not possible to directly show that there is supersymmetry enhancement via this mechanism, as was done in [27] for the theory with $d H=0$.

We have also constructed generalized Lichnerowicz type theorems, which relate spinors which are parallel with respect to a certain type of near-horizon supercovariant derivative, to zero modes of near-horizon Dirac operators. We have shown that if $\eta$ is a zero mode of the near-horizon Dirac operator to both zero and first order in $\alpha^{\prime}$, then the Lichnerowicz theorems imply that $\eta$ only satisfies the KSE (4.26) and (4.27) to zero order in $\alpha^{\prime}$. Hence, the types of arguments used to show supersymmetry enhancement via Lichnerowicz type theorems in [9-12] also do not work to the required order in $\alpha^{\prime}$ for the heterotic theory.

Finally, we have examined a class of nearly supersymmetric horizons for which the gravitino KSE is allowed to admit solutions on the spatial horizon section but not the rest of the KSEs. Such solutions in general do not admit any spacetime Killing spinors including solutions of the gravitino KSE. Under some conditions on the fluxes, we investigate the geometry of the spatial horizon sections using a combination of local and global techniques as well as the field equations. We find that those with a $G_{2}, \mathrm{SU}(3)$ and $\mathrm{SU}(2)$ structure admit 1, 2 and 4 parallel vectors on the spatial horizon sections with respect to the connection with torsion. The geometry on the orbit spaces of these isometries is fully specified.

The spacetime of both supersymmetric, and nearly supersymmetry horizons considered here admits a $\operatorname{SL}(2, \mathbb{R})$ symmetry at zeroth order in $\alpha^{\prime}$. In the supersymmetric case for which there is a $\eta_{-}$Killing spinor to order $\mathcal{O}\left(\alpha^{\prime 2}\right)$ such that $\eta_{-}$does not vanish at zeroth order, $\eta_{-}^{[0]} \neq 0$, this symmetry persists at first order in $\alpha^{\prime}$. The nearly supersymmetric horizons also admit an $\operatorname{SL}(2, \mathbb{R})$ symmetry provided that $h$ is parallel with respect to the connection with torsion up to $\mathcal{O}\left(\alpha^{\prime 2}\right)$.

It is not apparent whether the properties of the heterotic horizons described here are going to persist to higher than two loops in sigma model perturbation theory. It is likely though that the presence of an $\mathfrak{s l}(2, \mathbb{R})$ symmetry will persist after perhaps a suitable choice of a scheme in perturbation theory. There is no apparent reason to hypothesize that such a symmetry can be anomalous at higher loops. What happens to global properties of the horizons, for example the Lichnerowicz type theorems, is less clear. We have already seen that these theorems do not hold to the expected order in $\alpha^{\prime}$ even at two loops. This can be taken as an indication that additional higher order corrections may further weaken the consequences of such theorems.

\section{Acknowledgments}

AF is partially supported by the EPSRC grant FP/M506655. JG is supported by the STFC grant, ST/1004874/1. GP is partially supported by the STFC rolling grant ST/J002798/1.

Data management. No additional research data beyond the data presented and cited in this work are needed to validate the research findings in this work. 


\section{A Useful formulae}

\section{A.1 Spin connection and curvature}

In our conventions, the curvature of a connection $\Gamma$ is given by

$$
R_{A B}{ }^{C}{ }_{D}=\partial_{A} \Gamma_{B D}^{C}-\partial_{B} \Gamma_{A D}^{C}+\Gamma_{A N}^{C} \Gamma_{B D}^{N}-\Gamma_{B N}^{C} \Gamma_{A D}^{N} .
$$

We define connections $\check{\nabla}$ and $\hat{\nabla}$ as follows

$$
\check{\nabla}_{M} \xi^{N}=\nabla_{M} \xi^{N}-\frac{1}{2} H^{N}{ }_{M L} \xi^{L}, \quad \hat{\nabla}_{M} \xi^{N}=\nabla_{M} \xi^{N}+\frac{1}{2} H^{N}{ }_{M L} \xi^{L}
$$

for vector field $\xi$, where $\nabla$ is the Levi-Civita connection. In particular, the $\check{R}$ curvature tensor can be written as

$$
\check{R}_{A B, C D}=R_{A B C D}-\frac{1}{2} \nabla_{A} H_{C B D}+\frac{1}{2} \nabla_{B} H_{C A D}+\frac{1}{4} H_{C A N} H^{N}{ }_{B D}-\frac{1}{4} H_{C B N} H^{N}{ }_{A D},
$$

where $R$ is the Riemann curvature tensor. Also, note that

$$
\check{R}_{A B, C D}-\hat{R}_{C D, A B}=\frac{1}{2}(d H)_{A B C D} .
$$

We also define connections $\check{\tilde{\nabla}}$ and $\hat{\tilde{\nabla}}$ on the horizon section $\mathcal{S}$ via

$$
\tilde{\tilde{\nabla}}_{i} Y^{j}=\tilde{\nabla}_{i} Y^{j}-\frac{1}{2} W^{j}{ }_{i k} Y^{k}, \quad \hat{\tilde{\nabla}}_{i} Y^{j}=\tilde{\nabla}_{i} Y^{j}+\frac{1}{2} W^{j}{ }_{i k} Y^{k},
$$

for vector fields $Y$ on $\mathcal{S}$, and where $\tilde{\nabla}$ is the Levi-Civita connection of $\mathcal{S}$, and we denote the curvatures of the connections $\tilde{\nabla}, \check{\tilde{\nabla}}$ and $\hat{\tilde{\nabla}}$ by $\tilde{R}, \tilde{\tilde{R}}$ and $\hat{\tilde{R}}$ respectively.

The non-vanishing components of the spin connection in the frame basis (2.2) of the near horizon metric (2.1) are

$$
\begin{aligned}
& \Omega_{-,+i}=-\frac{1}{2} h_{i}, \quad \Omega_{+,+-}=-r \Delta, \quad \Omega_{+,+i}=\frac{1}{2} r^{2}\left(\Delta h_{i}-\partial_{i} \Delta\right), \\
& \Omega_{+,-i}=-\frac{1}{2} h_{i}, \quad \Omega_{+, i j}=-\frac{1}{2} r d h_{i j}, \quad \Omega_{i,+-}=\frac{1}{2} h_{i}, \quad \Omega_{i,+j}=-\frac{1}{2} r d h_{i j}, \\
& \Omega_{i, j k}=\tilde{\Omega}_{i, j k},
\end{aligned}
$$

where $\tilde{\Omega}$ denotes the spin-connection of the spatial horizon section $\mathcal{S}$ in the $\mathbf{e}^{i}$ basis. If $f$ is any function of spacetime, then frame derivatives are expressed in terms of co-ordinate derivatives as

$$
\partial_{+} f=\partial_{u} f+\frac{1}{2} r^{2} \Delta \partial_{r} f, \quad \partial_{-} f=\partial_{r} f, \quad \partial_{i} f=\tilde{\partial}_{i} f-r \partial_{r} f h_{i} .
$$

The non-vanishing components of the Ricci tensor is the basis (2.2) are

$$
\begin{aligned}
R_{+-} & =\frac{1}{2} \tilde{\nabla}^{i} h_{i}-\Delta-\frac{1}{2} h^{2}, \quad R_{i j}=\tilde{R}_{i j}+\tilde{\nabla}_{(i} h_{j)}-\frac{1}{2} h_{i} h_{j} \\
R_{++} & =r^{2}\left(\frac{1}{2} \tilde{\nabla}^{2} \Delta-\frac{3}{2} h^{i} \tilde{\nabla}_{i} \Delta-\frac{1}{2} \Delta \tilde{\nabla}^{i} h_{i}+\Delta h^{2}+\frac{1}{4}(d h)_{i j}(d h)^{i j}\right) \\
R_{+i} & =r\left(\frac{1}{2} \tilde{\nabla}^{j}(d h)_{i j}-(d h)_{i j} h^{j}-\tilde{\nabla}_{i} \Delta+\Delta h_{i}\right),
\end{aligned}
$$

where $\tilde{R}$ is the Ricci tensor of the horizon section $\mathcal{S}$ in the $\mathbf{e}^{i}$ frame. 
We remark that the non-vanishing components of the Hessian of $\Phi$, are given by

$$
\begin{aligned}
\nabla_{+} \nabla_{-} \Phi & =-\frac{1}{2} h^{i} \tilde{\nabla}_{i} \Phi, \\
\nabla_{+} \nabla_{i} \Phi & =-\frac{1}{2} r(d h)_{i}{ }^{j} \tilde{\nabla}_{j} \Phi, \\
\nabla_{i} \nabla_{j} \Phi & =\tilde{\nabla}_{i} \tilde{\nabla}_{j} \Phi,
\end{aligned}
$$

where in the above expression, we have set $\Delta=0$.

The non-vanishing components of the $\check{R}$ curvature tensor in the basis (2.2) are

$$
\begin{aligned}
\check{R}_{-i,+j} & =\tilde{\nabla}_{j} h_{i}+\frac{1}{2} h^{\ell} W_{\ell i j}, \quad \check{R}_{i j,+-}=d h_{i j}, \\
\check{R}_{i j,+k} & =r\left(\tilde{\nabla}_{k} d h_{i j}-h_{k} d h_{i j}+\frac{1}{2}(d h)_{i}{ }^{m} W_{m j k}-\frac{1}{2}(d h)_{j}{ }^{m} W_{m i k}\right), \\
\check{R}_{i j, k \ell} & =\tilde{R}_{i j k \ell}-\frac{1}{2} \tilde{\nabla}_{i} W_{k j \ell}+\frac{1}{2} \tilde{\nabla}_{j} W_{k i \ell}+\frac{1}{4} W_{k i m} W^{m}{ }_{j \ell}-\frac{1}{4} W_{k j m} W^{m}{ }_{i \ell} \\
& =\check{\tilde{R}}_{i j, k \ell},
\end{aligned}
$$

where in the above expression, we have set $\Delta=0, N=h$ and $Y=d h$. Note that the $\check{R}_{-i,+j}$ and $\check{R}_{i j,+k}$ terms give no contribution to the Bianchi identity of $H$ or to the Einstein equations, because $\check{R}_{M N,-i}=0$ for all $M, N$.

\section{A.2 Bosonic field equations}

The Bianchi identity associated with the 3 -form is

$$
d H=-\frac{\alpha^{\prime}}{4}(\operatorname{tr}(\check{R} \wedge \check{R})-\operatorname{tr}(F \wedge F))+\mathcal{O}\left(\alpha^{\prime 2}\right),
$$

where $\operatorname{tr}(F \wedge F)=F_{b}^{a} \wedge F_{a}^{b}(a, b$ are gauge indices on $F)$.

The Einstein equation is

$$
\begin{aligned}
R_{M N} & -\frac{1}{4} H_{M L_{1} L_{2}} H_{N}{ }^{L_{1} L_{2}}+2 \nabla_{M} \nabla_{N} \Phi \\
& +\frac{\alpha^{\prime}}{4}\left(\check{R}_{M L_{1}, L_{2} L_{3}} \check{R}_{N}{ }_{1} L_{2} L_{3}-F_{M L a b} F_{N}{ }^{L a b}\right)=\mathcal{O}\left(\alpha^{\prime 2}\right) .
\end{aligned}
$$

The gauge field equations are

$$
\nabla^{M}\left(e^{-2 \Phi} H_{M N_{1} N_{2}}\right)=\mathcal{O}\left(\alpha^{2}\right)
$$

and

$$
\nabla^{M}\left(e^{-2 \Phi} F_{M N}\right)+\frac{1}{2} e^{-2 \Phi} H_{N L_{1} L_{2}} F^{L_{1} L_{2}}=\mathcal{O}\left(\alpha^{\prime}\right) .
$$

The dilaton field equation is

$$
\begin{aligned}
\nabla_{M} \nabla^{M} \Phi= & 2 \nabla_{M} \Phi \nabla^{M} \Phi-\frac{1}{12} H_{N_{1} N_{2} N_{3}} H^{N_{1} N_{2} N_{3}} \\
& +\frac{\alpha^{\prime}}{16}\left(\check{R}_{N_{1} N_{2}, N_{3} N_{4}} \check{R}^{N_{1} N_{2}, N_{3} N_{4}}-F_{N_{1} N_{2} a b} F^{N_{1} N_{2} a b}\right)+\mathcal{O}\left(\alpha^{\prime 2}\right) .
\end{aligned}
$$

This completes the list of field equations. We have followed the conventions of [46]. 


\section{B Further simplification of the KSEs}

Here we shall show that the independent KSEs are given in (4.26) and (4.27). We first note that the conditions on the bosonic fields (3.18) (obtained from the case when $\phi_{+}^{[0]} \equiv 0$ ) actually imply those of (4.11) (corresponding to the $\phi_{+}^{[0]} \not \equiv 0$ case). Furthermore, the KSEs (3.19), (3.20), (3.21), (3.22), (3.23) and (3.24) are identical to the KSE (4.18), (4.20), (4.21), (3.22), (4.24) and (4.25). Hence, we shall concentrate on the simplification of the KSEs associated with the case $\phi_{+}^{[0]} \not \equiv 0$, as the simplification of the KSEs in the case $\phi_{+}^{[0]} \equiv 0$ follows in exactly the same way.

\section{B.1 Elimination of conditions (4.19), (4.21), (4.23), (4.25)}

Let us assume (4.18), (4.20), (4.22) and (4.24). Then acting on the algebraic conditions (4.20) and (4.24) with the Dirac operator $\Gamma^{\ell} \tilde{\nabla}_{\ell}$, one obtains

$$
\begin{aligned}
\left(\tilde{\nabla}_{i} \tilde{\nabla}^{i} \Phi\right. & \mp h^{i} \tilde{\nabla}_{i} \Phi-2 \tilde{\nabla}^{i} \Phi \tilde{\nabla}_{i} \Phi-\frac{1}{2} h_{i} h^{i}+\frac{1}{12} W_{i j k} W^{i j k}+\frac{1}{4}(1 \pm 1) d h_{i j} \Gamma^{i j} \\
+ & \left.\frac{1}{4}(-1 \pm 1) h^{k} W_{k i j} \Gamma^{i j}-\frac{1}{48} d W_{\ell_{1} \ell_{2} \ell_{3} \ell_{4}} \Gamma^{\ell_{1} \ell_{2} \ell_{3} \ell_{4}}\right) \phi_{ \pm}=\mathcal{O}\left(\alpha^{\prime 2}\right)
\end{aligned}
$$

where we have made use of the field equations (4.13) and (4.15), together with the algebraic conditions (4.20) and (4.24). Next, on substituting the dilaton equation and the Bianchi identity into the above expression, one finds

$$
\begin{aligned}
& \left(\frac{1}{2}(1 \mp 1) \tilde{\nabla}^{i} h_{i}+\frac{1}{4}(1 \pm 1) d h_{i j} \Gamma^{i j}+\frac{1}{4}(-1 \pm 1)\left(i_{h} W\right)_{i j} \Gamma^{i j}\right. \\
& \left.\quad+\frac{\alpha^{\prime}}{32}\left(2 d h_{i j} \Gamma^{i j} d h_{p q} \Gamma^{p q}+\tilde{F}_{i j}{ }^{a b} \Gamma^{i j} \tilde{F}_{p q a b} \Gamma^{p q}-\check{\tilde{R}}_{i j,}{ }^{m n} \Gamma^{i j} \tilde{\tilde{R}}_{p q, m n} \Gamma^{p q}\right)\right) \phi_{ \pm}=\mathcal{O}\left(\alpha^{\prime 2}\right) .
\end{aligned}
$$

Further simplification can be obtained by noting that the integrability conditions of the KSE (4.18) and (4.22) are

$$
\hat{\tilde{R}}_{i j, p q} \Gamma^{p q} \phi_{ \pm}=\mathcal{O}\left(\alpha^{2}\right)
$$

and hence

$$
\check{\tilde{R}}_{p q, i j} \Gamma^{p q} \phi_{ \pm}=\mathcal{O}\left(\alpha^{\prime}\right),
$$

from which it follows that the final term on the r.h.s. of (B.2) is $\mathcal{O}\left(\alpha^{\prime 2}\right)$ and hence can be neglected. So, (B.2) is equivalent to

$$
\begin{aligned}
\left(\frac{1}{2}(1 \mp 1) \tilde{\nabla}^{i} h_{i}\right. & +\frac{1}{4}(1 \pm 1) d h_{i j} \Gamma^{i j}+\frac{1}{4}(-1 \pm 1)\left(i_{h} W\right)_{i j} \Gamma^{i j} \\
& \left.+\frac{\alpha^{\prime}}{16} d h_{i j} \Gamma^{i j} d h_{p q} \Gamma^{p q}+\frac{\alpha^{\prime}}{32} \tilde{F}_{i j}^{a b} \Gamma^{i j} \tilde{F}_{p q a b} \Gamma^{p q}\right) \phi_{ \pm}=\mathcal{O}\left(\alpha^{\prime 2}\right) .
\end{aligned}
$$

We begin by considering the condition which (B.5) imposes on $\phi_{+}$:

$$
\left(\frac{1}{2} d h_{i j} \Gamma^{i j}+\frac{\alpha^{\prime}}{16} d h_{i j} \Gamma^{i j} d h_{p q} \Gamma^{p q}+\frac{\alpha^{\prime}}{32} \tilde{F}_{i j}^{a b} \Gamma^{i j} \tilde{F}_{p q a b} \Gamma^{p q}\right) \phi_{+}=\mathcal{O}\left(\alpha^{\prime 2}\right) .
$$


To zeroth order this gives

$$
d h_{i j} \Gamma^{i j} \phi_{+}=\mathcal{O}\left(\alpha^{\prime}\right)
$$

which implies that the second term on the 1.h.s. of (B.6) is of $\mathcal{O}\left(\alpha^{2}\right)$, and hence can be neglected. Using this, (B.6) gives that

$$
\alpha^{\prime}\left\langle\tilde{F}_{i j}^{a b} \Gamma^{i j} \phi_{+}, \tilde{F}_{p q a b} \Gamma^{p q} \phi_{+}\right\rangle=\mathcal{O}\left(\alpha^{\prime 2}\right)
$$

which implies that

$$
\tilde{F}_{i j}{ }^{a b} \Gamma^{i j} \phi_{+}=\mathcal{O}\left(\alpha^{\prime}\right)
$$

Using this the third term on the l.h.s. of (B.6) is also of $\mathcal{O}\left(\alpha^{2}\right)$. So, the remaining content of (B.6) is

$$
d h_{i j} \Gamma^{i j} \phi_{+}=\mathcal{O}\left(\alpha^{\prime 2}\right)
$$

Hence, we have proven that the KSE (4.18) and (4.20) imply the algebraic KSE (4.19) and (4.21).

Next, we consider the condition which (B.5) imposes on $\phi_{-}$, which is

$$
\left(\tilde{\nabla}^{i} h_{i}-\frac{1}{2}\left(i_{h} W\right)_{i j} \Gamma^{i j}+\frac{\alpha^{\prime}}{32}\left(2 d h_{i j} \Gamma^{i j} d h_{p q} \Gamma^{p q}+\tilde{F}_{i j}{ }^{a b} \Gamma^{i j} \tilde{F}_{p q a b} \Gamma^{p q}\right)\right) \phi_{-}=\mathcal{O}\left(\alpha^{\prime 2}\right)
$$

However, note also that the $u$-dependent part of (4.18), with (4.22), implies that

$$
\left(\tilde{\nabla}_{i} h_{j}-\frac{1}{2} W_{i j k} h^{k}\right) \Gamma^{j} \phi_{-}=\mathcal{O}\left(\alpha^{\prime 2}\right)
$$

On contracting this expression with $\Gamma^{i}$, we find

$$
\left(\tilde{\nabla}^{i} h_{i}+\frac{1}{2} d h_{i j} \Gamma^{i j}-\frac{1}{2}\left(i_{h} W\right)_{i j} \Gamma^{i j}\right) \phi_{-}=\mathcal{O}\left(\alpha^{2}\right),
$$

and on substituting this expression into (B.11) we get

$$
\left(-\frac{1}{2} d h_{i j} \Gamma^{i j}+\frac{\alpha^{\prime}}{16} d h_{i j} \Gamma^{i j} d h_{p q} \Gamma^{p q}+\frac{\alpha^{\prime}}{32} \tilde{F}_{i j}^{a b} \Gamma^{i j} \tilde{F}_{p q a b} \Gamma^{p q}\right) \phi_{-}=\mathcal{O}\left(\alpha^{\prime 2}\right)
$$

Hence, we find from exactly the same reasoning which was used to analyse the conditions on $\phi_{+}$, that (4.22) and (4.24) imply (4.23) and (4.25).

So, on making use of the field equations, it follows that the necessary and sufficient conditions for supersymmetry simplify to the conditions (4.18) and (4.20) on $\phi_{+}$, and to (4.22) and (4.24) on $\eta_{-}$. We remark that the $u$-dependent parts of the conditions (4.18) and (4.20) also impose conditions on $\eta_{-}$. We shall examine the conditions on $\eta_{-}$further in the next section, and show how these may be simplified. 


\section{B.2 Elimination of $u$-dependent parts of (4.18) and (4.20)}

We begin by considering the $u$-dependent parts of (4.18) and (4.20), assuming that (4.22) and (4.24) hold. The $u$-dependent part of the condition on $\phi_{+}$obtained from (4.18) is

$$
\left(\tilde{\nabla}_{i} h_{j}-\frac{1}{2} W_{i j k} h^{k}\right) \Gamma^{j} \eta_{-}=\mathcal{O}\left(\alpha^{\prime 2}\right),
$$

and the $u$-dependent part of the algebraic condition (4.20) is given by

$$
\left(\Gamma^{i} \tilde{\nabla}_{i} \Phi+\frac{1}{2} h_{i} \Gamma^{i}-\frac{1}{12} W_{i j k} \Gamma^{i j k}\right) h_{\ell} \Gamma^{\ell} \eta_{-}=\mathcal{O}\left(\alpha^{\prime 2}\right) .
$$

On adding $h_{\ell} \Gamma^{\ell}$ acting on (4.24) to the above expression, we find that (B.16) is equivalent to the condition

$$
\left(\tilde{\nabla}^{i} h_{i}-\frac{1}{2} h^{i} W_{i j k} \Gamma^{j k}\right) \eta_{-}=\mathcal{O}\left(\alpha^{\prime 2}\right),
$$

where we have also made use of the field equation (4.13). On contracting (B.15) with $\Gamma^{i}$, it then follows that (B.17) is equivalent to

$$
d h_{i j} \Gamma^{i j} \eta_{-}=\mathcal{O}\left(\alpha^{\prime 2}\right) .
$$

However, as shown in the previous section, this condition is implied by (4.22) and (4.24) on making use of the field equations.

So, it remains to consider the condition (B.15). First, recall that the integrability conditions of the gravitino equation of (4.22) is given by

$$
\hat{\tilde{R}}_{i j, k \ell} \Gamma^{k \ell} \eta_{-}=\mathcal{O}\left(\alpha^{\prime 2}\right) .
$$

On contracting with $\Gamma^{j}$, one then obtains

$$
\begin{aligned}
& \left(\left(-2 \tilde{R}_{i j}+\frac{1}{2} W_{i m n} W_{j}^{m n}-2 \tilde{\nabla}^{k} \Phi W_{k i j}+d h_{i j}-h^{k} W_{k i j}\right) \Gamma^{j}\right. \\
& \left.\quad+\left(-\frac{1}{6}(d W)_{i j k \ell}-\frac{1}{3} \tilde{\nabla}_{i} W_{j k \ell}+\frac{1}{2} W_{i j}{ }^{m} W_{k \ell m}\right) \Gamma^{j k \ell}\right) \eta_{-}=\mathcal{O}\left(\alpha^{\prime 2}\right),
\end{aligned}
$$

where we have used the gauge equation (4.15). Also, on taking the covariant derivative of the algebraic condition (4.24), and using (4.22), one also finds the following mixed integrability condition

$$
\begin{aligned}
& \left(\left(\tilde{\nabla}_{i} \tilde{\nabla}_{j} \Phi-\frac{1}{2} \tilde{\nabla}_{i} h_{j}+\frac{1}{2} W_{i k j} \tilde{\nabla}^{k} \Phi-\frac{1}{4} W_{i k j} h^{k}\right) \Gamma^{j}\right. \\
& \left.\quad+\Gamma^{j k \ell}\left(-\frac{1}{12} \tilde{\nabla}_{i} W_{j k \ell}+\frac{1}{8} W_{j k m} W_{i \ell}{ }^{m}\right)\right) \eta_{-}=\mathcal{O}\left(\alpha^{\prime 2}\right) .
\end{aligned}
$$

On eliminating the $\tilde{\nabla}_{i} W_{j k \ell} \Gamma^{j k \ell}$ terms between (B.20) and (B.21), one obtains the condition

$$
\begin{aligned}
\left(\left(-2 \tilde{R}_{i j}+\frac{1}{2} W_{i m n} W_{j}^{m n}+d h_{i j}-2 h^{k} W_{k i j}\right.\right. & \left.-4 \tilde{\nabla}_{i} \tilde{\nabla}_{j} \Phi+2 \tilde{\nabla}_{i} h_{j}\right) \Gamma^{j} \\
& \left.-\frac{1}{6} d W_{i j k \ell} \Gamma^{j k \ell}\right) \eta_{-}=\mathcal{O}\left(\alpha^{\prime 2}\right) .
\end{aligned}
$$


Next, we substitute the Einstein equation (4.16) in order to eliminate the Ricci tensor, and also use the Bianchi identity for $d W$. One then obtains, after some rearrangement of terms, the following condition

$$
\begin{aligned}
\left(\left(4 \tilde{\nabla}_{i} h_{j}-2 h^{k} W_{k i j}\right) \Gamma^{j}+\alpha^{\prime}(\right. & \frac{1}{2} d h_{i j} \Gamma^{j} d h_{k \ell} \Gamma^{k \ell}+\frac{1}{4} \tilde{F}_{i j a b} \Gamma^{j} \tilde{F}_{k \ell}{ }^{a b} \Gamma^{k \ell} \\
& \left.\left.-\frac{1}{4} \tilde{\tilde{R}}_{i j,}{ }^{m n} \Gamma^{j} \tilde{\tilde{R}}_{k \ell, m n} \Gamma^{k \ell}\right)\right) \eta_{-}=\mathcal{O}\left(\alpha^{\prime 2}\right) .
\end{aligned}
$$

The $\alpha^{\prime}$ terms in the above expression can be neglected, as they all give rise to terms which are in fact $\mathcal{O}\left(\alpha^{\prime 2}\right)$. This is because of the conditions (4.23) and (4.25), which we have already shown follow from (4.22) and (4.24), together with the bosonic conditions, as well as the fact that

$$
\check{\tilde{R}}_{k \ell, m n} \Gamma^{k \ell} \eta_{-}=\mathcal{O}\left(\alpha^{\prime}\right),
$$

which follows from the integrability condition of (4.22). It follows that (B.23) implies (B.15).

\section{A consistency condition}

Suppose that we consider the Bianchi identity associated with the 3-form as

$$
d H=-\frac{\alpha^{\prime}}{4}(\operatorname{tr}(\mathcal{R} \wedge \mathcal{R})-\operatorname{tr}(F \wedge F))+\mathcal{O}\left(\alpha^{\prime 2}\right),
$$

where $\mathcal{R}$ is a spacetime curvature which will be specified later. Also observe that the 2 -form gauge potential and the Einstein equation can be written together as

$$
\hat{R}_{M N}+2 \hat{\nabla}_{M} \nabla_{N} \Phi+\frac{\alpha^{\prime}}{4}\left(\mathcal{R}_{M L_{1}, L_{2} L_{3}} \mathcal{R}_{N}^{L_{1}, L_{2} L_{3}}-F_{M L a b} F_{N}{ }^{L a b}\right)=\mathcal{O}\left(\alpha^{\prime 2}\right) .
$$

Then one can establish by direct computation that

$$
\hat{R}_{M[N, P Q]}=-\frac{1}{3} \hat{\nabla}_{M} H_{N P Q}-\frac{1}{6} d H_{M N P Q} .
$$

Using this and the field equations of the theory, one can derive the relation

$$
\begin{aligned}
\hat{R}_{M N, P Q} \Gamma^{N} \Gamma^{P Q} \epsilon= & -\frac{1}{3} \hat{\nabla}_{M}\left(H_{L P Q} \Gamma^{L P Q}-12 \partial_{L} \Phi \Gamma^{L}\right) \epsilon \\
& -\frac{\alpha^{\prime}}{4}\left[\mathcal{R}_{M N, E F} \mathcal{R}_{P Q},{ }^{E F}-F_{M N a b} F_{P Q}{ }^{a b}\right] \Gamma^{N} \Gamma^{P Q} \epsilon+\mathcal{O}\left(\alpha^{\prime 2}\right)
\end{aligned}
$$

If $\epsilon$ satisfies that gravitino KSE, the left hand side of this relation vanishes. Furthermore the right-hand-side vanishes as well provided that dilatino and gaugino KSEs are satisfied, and in addition

$$
\mathcal{R}_{P Q}{ }^{E F} \Gamma^{P Q} \epsilon=\mathcal{O}\left(\alpha^{\prime}\right) .
$$

Of course in heterotic string perturbation theory

$$
\check{R}_{P Q}{ }^{E F} \Gamma^{P Q} \epsilon=\mathcal{O}\left(\alpha^{\prime}\right)
$$


as a consequence of the gravitino KSE and the closure of $H$ at that order. Thus one can set $\mathcal{R}=\check{R}$ and the identity (C.4) will hold up to order $\alpha^{\prime 2}$.

One consequence of the identity (C.4) is that if the gravitino KSE and gaugino KSEs are satisfied as well as (C.5) but the dilatino is not, then the gravitino KSE admits an additional parallel spinor of the opposite chirality. Such kind of identities have been established before for special cases in [42]. Here we have shown that such a result is generic in the context of heterotic theory.

\section{Lichnerowicz theorem computation}

In this appendix, we present the details for the calculation of the functional $\mathcal{I}$ defined in (7.15), and show how the constants $q$ and $c$ are fixed by requiring that certain types of terms which arise in the calculation should vanish. We begin by considering the calculation at zeroth order in $\alpha^{\prime}$, and then include the corrections at first order in $\alpha^{\prime}$. We remark that we shall retain terms of the type $h^{i} \tilde{\nabla}_{i} \Phi$ throughout. This is because although these terms vanish at zeroth order in $\alpha^{\prime}$ as a consequence of the analysis in section 8 , it does not follow from this analysis that $\mathcal{L}_{h} \Phi=\mathcal{O}\left(\alpha^{\prime 2}\right)$. However, as we shall see, it turns out that the coefficient multiplying the terms $h^{i} \tilde{\nabla}_{i} \Phi$, which depends on the constants $q$ and $c$, vanishes when one requires that several other terms in $\mathcal{I}$ vanish as well. So these terms do not give any contribution to $\mathcal{I}$ at either zeroth or first order in $\alpha^{\prime}$.

\section{D.1 Computations at zeroth order in $\alpha^{\prime}$}

Throughout the following analysis, we assume Einstein equations, dilaton field equation and Bianchi identity at zeroth order in $\alpha^{\prime}$. To proceed, we expand out the definition of $\nabla_{i}^{(\kappa)}$ and $\mathcal{D}$ in $\mathcal{I}$, obtaining the following expression

$$
\begin{gathered}
\mathcal{I}=\int_{\mathcal{S}} e^{c \Phi} 2(\kappa-q)\left\langle\Gamma^{i} \mathcal{A} \eta_{ \pm}, \hat{\tilde{\nabla}}_{i} \eta_{ \pm}\right\rangle+e^{c \Phi}\left(8 \kappa^{2}-q^{2}\right)\left\langle\eta_{ \pm}, \mathcal{A}^{\dagger} \mathcal{A} \eta_{ \pm}\right\rangle \\
-e^{c \Phi}\left\langle\hat{\tilde{\nabla}}_{i} \eta_{ \pm}, \Gamma^{i j} \hat{\tilde{\nabla}}_{j} \eta_{ \pm}\right\rangle
\end{gathered}
$$

Now, after writing $\hat{\tilde{\nabla}}$ in terms of the Levi-Civita connection $\tilde{\nabla}$ and after integrating by parts, the expression (D.1) decomposes into

$$
\mathcal{I}=\mathcal{I}_{1}+\mathcal{I}_{2}+\mathcal{I}_{3},
$$

where

$$
\begin{gathered}
\mathcal{I}_{1}=\int_{\mathcal{S}} e^{c \Phi} 2(\kappa-q)\left\langle\eta_{ \pm}, \mathcal{A}^{\dagger} \mathcal{D} \eta_{ \pm}\right\rangle+e^{c \Phi}\left(8 \kappa^{2}-2 \kappa q+q^{2}\right)\left\langle\eta_{ \pm}, \mathcal{A}^{\dagger} \mathcal{A} \eta_{ \pm}\right\rangle \\
-\frac{1}{64} e^{c \Phi}\left\langle\eta_{ \pm}, \Gamma^{\ell_{1} \ell_{2}} \Gamma^{i j} \Gamma^{\ell_{3} \ell_{4}} W_{i \ell_{1} \ell_{2}} W_{j \ell_{3} \ell_{4}} \eta_{ \pm}\right\rangle
\end{gathered}
$$

and

$$
\begin{gathered}
\mathcal{I}_{2}=\int_{\mathcal{S}} c e^{c \Phi}\left\langle\eta_{ \pm}, \Gamma^{i j} \tilde{\nabla}_{j} \eta_{ \pm}\right\rangle+\frac{1}{8} e^{c \Phi}\left\langle\tilde{\nabla}_{i} \eta_{ \pm}, \Gamma^{i j} \Gamma^{\ell_{1} \ell_{2}} W_{j \ell_{1} \ell_{2}} \eta_{ \pm}\right\rangle \\
-\frac{1}{8} e^{c \Phi}\left\langle\eta_{ \pm}, \Gamma^{\ell_{1} \ell_{2}} \Gamma^{i j} W_{j \ell_{1} \ell_{2}} \tilde{\nabla} \eta_{ \pm}\right\rangle
\end{gathered}
$$


and

$$
\mathcal{I}_{3}=\int_{\mathcal{S}}-e^{c \Phi}\left\langle\tilde{\nabla}_{i} \eta_{ \pm}, \Gamma^{i j} \tilde{\nabla}_{j} \eta_{ \pm}\right\rangle
$$

In particular, we note the identity

$$
\Gamma^{\ell_{1} \ell_{2}} \Gamma^{i j} \Gamma^{\ell_{3} \ell_{4}} W_{i \ell_{1} \ell_{2}} W_{j \ell_{3} \ell_{4}}=8 W^{i} \ell_{1} \ell_{2} W_{i \ell_{3} \ell_{4}} \Gamma^{\ell_{1} \ell_{2} \ell_{3} \ell_{4}}-4 W_{i j k} W^{i j k}
$$

which simplifies $\mathcal{I}_{1}$. After integrating by parts the second term in $\mathcal{I}_{2}$, we have

$$
\begin{aligned}
\mathcal{I}_{2}= & \int_{\mathcal{S}} c e^{c \Phi}\left\langle\eta_{ \pm}, \Gamma^{i j} \tilde{\nabla}_{j} \eta_{ \pm}\right\rangle-\frac{1}{8} e^{c \Phi}\left\langle\eta_{ \pm},\left(\Gamma^{i j} \Gamma_{m n}-\Gamma_{m n} \Gamma^{i j}\right) W_{j}^{m n} \tilde{\nabla}_{i} \eta_{ \pm}\right\rangle \\
& -\frac{c}{8} e^{c \Phi}\left\langle\eta_{ \pm}, \Gamma^{i \ell_{1} \ell_{2} \ell_{3}} \tilde{\nabla}_{i} \Phi W_{\ell_{1} \ell_{2} \ell_{3}} \eta_{ \pm}\right\rangle-\frac{1}{8} e^{c \Phi}\left\langle\eta_{ \pm}, \Gamma^{\ell_{1} \ell_{2} \ell_{3} \ell_{4}} \tilde{\nabla}_{\ell_{1}} W_{\ell_{2} \ell_{3} \ell_{4}} \eta_{ \pm}\right\rangle
\end{aligned}
$$

where the last term is order $\alpha^{\prime}$, so we shall neglect it. Now we shall focus on the second term of (D.8). First note that

$$
\left(\Gamma^{i j} \Gamma_{m n}-\Gamma_{m n} \Gamma^{i j}\right) W_{j}^{m n}=-4 \Gamma^{m n} W_{m n}^{i}=\frac{4}{3} W_{\ell_{1} \ell_{2} \ell_{3}}\left(\Gamma^{\ell_{1} \ell_{2} \ell_{3}} \Gamma^{i}+\Gamma^{i \ell_{1} \ell_{2} \ell_{3}}\right) .
$$

Then, after an integration by parts and after writing $\tilde{\nabla}$ in terms of $\mathcal{D}$, we have

$$
\begin{aligned}
\int_{\mathcal{S}}- & \frac{1}{8} e^{c \Phi}\left\langle\eta_{ \pm},\left(\Gamma^{i j} \Gamma_{m n}-\Gamma_{m n} \Gamma^{i j}\right) W_{j}^{m n} \tilde{\nabla}_{i} \eta_{ \pm}\right\rangle=\int_{\mathcal{S}}-\frac{1}{6} e^{c \Phi}\left\langle\eta_{ \pm}, W_{\ell_{1} \ell_{2} \ell_{3}} \Gamma^{\ell_{1} \ell_{2} \ell_{3}} \mathcal{D} \eta_{ \pm}\right\rangle \\
& +\frac{q}{6} e^{c \Phi}\left\langle\eta_{ \pm}, W_{\ell_{1} \ell_{2} \ell_{3}} \Gamma^{\ell_{1} \ell_{2} \ell_{3}} \mathcal{A} \eta_{ \pm}\right\rangle-\frac{1}{48} e^{c \Phi}\left\langle\eta_{ \pm}, W_{\ell_{1} \ell_{2} \ell_{3}} \Gamma^{\ell_{1} \ell_{2} \ell_{3}} W_{i j k} \Gamma^{i j k} \eta_{ \pm}\right\rangle \\
& +\frac{c}{12} e^{c \Phi}\left\langle\eta_{ \pm}, \Gamma^{i \ell_{1} \ell_{2} \ell_{3}} \tilde{\nabla}_{i} \Phi W_{\ell_{1} \ell_{2} \ell_{3}} \eta_{ \pm}\right\rangle+\frac{1}{12} e^{c \Phi}\left\langle\eta_{ \pm}, \Gamma^{\ell_{1} \ell_{2} \ell_{3} \ell_{4}} \tilde{\nabla}_{\ell_{1}} W_{\ell_{2} \ell_{3} \ell_{4}} \eta_{ \pm}\right\rangle
\end{aligned}
$$

The last term of (D.10) is order $\alpha^{\prime}$, so we shall neglect it. To proceed further, we shall substitute $W_{i j k} \Gamma^{i j k}$ in terms of $\mathcal{A}$, using its definition. This produces terms proportional to the norm squared of $\mathcal{A} \eta_{ \pm}$, together with a number of counterterms. In detail, one obtains

$$
\begin{aligned}
\int_{\mathcal{S}}- & \frac{1}{8} e^{c \Phi}\left\langle\eta_{ \pm},\left(\Gamma^{i j} \Gamma_{m n}-\Gamma_{m n} \Gamma^{i j}\right) W_{j}{ }^{m n} \tilde{\nabla}_{i} \eta_{ \pm}\right\rangle=\int_{\mathcal{S}}-\frac{1}{6} e^{c \Phi}\left\langle\eta_{ \pm}, W_{\ell_{1} \ell_{2} \ell_{3}} \Gamma^{\ell_{1} \ell_{2} \ell_{3}} \mathcal{D} \eta_{ \pm}\right\rangle \\
& +e^{c \Phi}\left(\frac{1}{48}-\frac{q}{6}\right)\left\langle\eta_{ \pm}, \mathcal{A}^{\dagger} \mathcal{A} \eta_{ \pm}\right\rangle+e^{c \Phi}\left(\frac{1}{2}-2 q\right)\left\langle\eta_{ \pm}, \Gamma^{i} \tilde{\nabla}_{i} \Phi \mathcal{A} \eta_{ \pm}\right\rangle \\
& \pm e^{c \Phi}\left(\frac{1}{4}-q\right)\left\langle\eta_{ \pm}, \Gamma^{i} h_{i} \mathcal{A} \eta_{ \pm}\right\rangle+3 e^{c \Phi}\left\langle\eta_{ \pm}, \tilde{\nabla}_{i} \Phi \tilde{\nabla}^{i} \Phi \eta_{ \pm}\right\rangle \pm 3 e^{c \Phi}\left\langle\eta_{ \pm}, h^{i} \tilde{\nabla}_{i} \Phi \eta_{ \pm}\right\rangle \\
& +\frac{3}{4} e^{c \Phi}\left\langle\eta_{ \pm}, h_{i} h^{i} \eta_{ \pm}\right\rangle+\frac{c}{12} e^{c \Phi}\left\langle\eta_{ \pm}, \Gamma^{i \ell_{1} \ell_{2} \ell_{3}} \tilde{\nabla}_{i} \Phi W_{\ell_{1} \ell_{2} \ell_{3}} \eta_{ \pm}\right\rangle+\mathcal{O}\left(\alpha^{\prime}\right)
\end{aligned}
$$

Let us focus now on the first term of (D.8). After writing $\Gamma^{i j}$ as $\Gamma^{i} \Gamma^{j}-\delta^{i j}$ and after integrating by parts, we have

$$
\begin{aligned}
\int_{\mathcal{S}} c e^{c \Phi}\left\langle\eta_{ \pm}, \Gamma^{i j} \tilde{\nabla}_{j} \eta_{ \pm}\right\rangle= & \int_{\mathcal{S}} c e^{c \Phi}\left\langle\eta_{ \pm}, \Gamma^{\ell} \tilde{\nabla}_{\ell} \Phi \Gamma^{i} \tilde{\nabla}_{i} \eta_{ \pm}\right\rangle \\
& +\frac{c}{2} e^{c \Phi}\left\langle\eta_{ \pm}, \tilde{\nabla}_{i} \tilde{\nabla}^{i} \Phi \eta_{ \pm}\right\rangle+\frac{c^{2}}{2} e^{c \Phi}\left\langle\eta_{ \pm}, \tilde{\nabla}_{i} \Phi \tilde{\nabla}^{i} \Phi \eta_{ \pm}\right\rangle .
\end{aligned}
$$


The first term in the r.h.s. of (D.12) can be rewritten in terms of the modified Dirac operator $\mathcal{D}$ after subtracting suitable terms. The second term on the r.h.s. can be further simplified using the dilaton field equation at zeroth order in $\alpha^{\prime}$. On performing these calculations, we have

$$
\begin{aligned}
& \int_{\mathcal{S}} c e^{c \Phi}\left\langle\eta_{ \pm}, \Gamma^{i j} \tilde{\nabla}_{j} \eta_{ \pm}\right\rangle=\int_{\mathcal{S}} c e^{c \Phi}\left\langle\eta_{ \pm}, \Gamma^{\ell} \tilde{\nabla}_{\ell} \Phi \mathcal{D} \eta_{ \pm}\right\rangle-\frac{c}{24} e^{c \Phi}\left\langle\eta_{ \pm}, W_{i j k} W^{i j k} \eta_{ \pm}\right\rangle \\
& +c\left(\frac{1}{8}-q\right) e^{c \Phi}\left\langle\eta_{ \pm}, \Gamma^{i \ell_{1} \ell_{2} \ell_{3}} \tilde{\nabla}_{i} \Phi W_{\ell_{1} \ell_{2} \ell_{3}} \eta_{ \pm}\right\rangle+\frac{c}{4} e^{c \Phi}\left\langle\eta_{ \pm}, h_{i} h^{i} \eta_{ \pm}\right\rangle \\
& +12 c\left(\frac{1}{12}+\frac{c}{24}+q\right) e^{c \Phi}\left\langle\eta_{ \pm}, \tilde{\nabla}_{i} \Phi \tilde{\nabla}^{i} \Phi \eta_{ \pm}\right\rangle \\
& +6 c\left(\frac{1}{12} \pm q\right) e^{c \Phi}\left\langle\eta_{ \pm}, h^{i} \tilde{\nabla}_{i} \Phi \eta_{ \pm}\right\rangle+\mathcal{O}\left(\alpha^{\prime}\right) \text {. }
\end{aligned}
$$

Let us now focus on $\mathcal{I}_{3}$. Recall that

$$
\Gamma^{i j} \tilde{\nabla}_{i} \tilde{\nabla}_{j} \eta_{ \pm}=-\frac{1}{4} \tilde{R} \eta_{ \pm}
$$

Therefore after integrating by parts and using (D.14) neglecting $\alpha^{\prime}$ corrections from Einstein equations, $\mathcal{I}_{3}$ becomes

$$
\begin{aligned}
\mathcal{I}_{3}=\int_{\mathcal{S}}- & \frac{5}{48} e^{c \Phi}\left\langle\eta_{ \pm}, W_{i j k} W^{i j k} \eta_{ \pm}\right\rangle+e^{c \Phi}\left\langle\eta_{ \pm}, \tilde{\nabla}_{i} \Phi \tilde{\nabla}^{i} \Phi \eta_{ \pm}\right\rangle+\frac{1}{4} e^{c \Phi}\left\langle\eta_{ \pm}, h_{i} h^{i} \eta_{ \pm}\right\rangle \\
& +e^{c \Phi}\left\langle\eta_{ \pm}, h^{i} \tilde{\nabla}_{i} \Phi \eta_{ \pm}\right\rangle+\mathcal{O}\left(\alpha^{\prime}\right) .
\end{aligned}
$$

Collecting together all terms and substituting $h_{i} h^{i}$ by inverting the zeroth order in $\alpha^{\prime}$ dilaton filed equation, one finally gets

$$
\begin{aligned}
\mathcal{I}=\int_{\mathcal{S}} e^{c \Phi}\left\langle\eta_{ \pm},\left(c \Gamma^{\ell} \tilde{\nabla}_{\ell} \Phi-\frac{1}{6} W_{\ell_{1} \ell_{2} \ell_{3}} \Gamma^{\ell_{1} \ell_{2} \ell_{3}}+2(\kappa-q) \mathcal{A}^{\dagger}\right) \mathcal{D} \eta_{ \pm}\right\rangle \\
+\left(8 \kappa^{2}-2 \kappa q-\frac{q}{12}+q^{2}\right) e^{c \Phi}\left\langle\eta_{ \pm}, \mathcal{A}^{\dagger} \mathcal{A} \eta_{ \pm}\right\rangle \\
+\frac{3}{4}\left(q-\frac{1}{12}\right) e^{c \Phi}\left\langle\eta_{ \pm}, W^{i}{ }_{\ell_{1} \ell_{2}} W^{i \ell_{3} \ell_{4}} \Gamma^{\ell_{1} \ell_{2} \ell_{3} \ell_{4}} \eta_{ \pm}\right\rangle \\
\quad-c\left(q-\frac{1}{12}\right) e^{c \Phi}\left\langle\eta_{ \pm}, \Gamma^{i \ell_{1} \ell_{2} \ell_{3}} \tilde{\nabla}_{i} \Phi W_{\ell_{1} \ell_{2} \ell_{3}} \eta_{ \pm}\right\rangle \\
+6\left(\frac{1}{12}+q+\frac{c}{12}\right) e^{c \Phi}\left\langle\eta_{ \pm}, \tilde{\nabla}_{i} \tilde{\nabla}^{i} \Phi \eta_{ \pm}\right\rangle \\
+12 c\left(q+\frac{c}{24}\right) e^{c \Phi}\left\langle\eta_{ \pm}, \tilde{\nabla}_{i} \Phi \tilde{\nabla}^{i} \Phi \eta_{ \pm}\right\rangle \\
+\left(\frac{1}{2}-6 q \pm 6 q(c+2)\right) e^{c \Phi}\left\langle\eta_{ \pm}, h^{i} \tilde{\nabla}_{i} \Phi \eta_{ \pm}\right\rangle+\mathcal{O}\left(\alpha^{\prime}\right) .
\end{aligned}
$$

In order to eliminate the term $\left\langle\eta_{ \pm}, W^{i} \ell_{1} \ell_{2} W_{i \ell_{3} \ell_{4}} \Gamma^{\ell_{1} \ell_{2} \ell_{3} \ell_{4}} \eta_{ \pm}\right\rangle$, which has no sign and cannot be rewritten in terms of $\mathcal{D}$ or $\mathcal{A}^{\dagger} \mathcal{A}$, we must set

$$
q=\frac{1}{12}+\mathcal{O}\left(\alpha^{\prime}\right)
$$


and then in order to eliminate the $\left\langle\eta_{ \pm}, \tilde{\nabla}^{i} \tilde{\nabla}_{i} \Phi \eta_{ \pm}\right\rangle$term we must further set

$$
c=-2+\mathcal{O}\left(\alpha^{\prime}\right)
$$

Then (D.16) simplifies to

$$
\mathcal{I}=\int_{\mathcal{S}} e^{-2 \Phi}\left\langle\eta_{ \pm}, \Psi \mathcal{D} \eta_{ \pm}\right\rangle+\left(8 \kappa^{2}-\frac{\kappa}{6}\right) \int_{\mathcal{S}} e^{-2 \Phi}\left\|\mathcal{A} \eta_{ \pm}\right\|^{2}+\mathcal{O}\left(\alpha^{\prime}\right)
$$

where

$$
\Psi \equiv-2 \Gamma^{\ell} \tilde{\nabla}_{\ell} \Phi-\frac{1}{6} W_{\ell_{1} \ell_{2} \ell_{3}} \Gamma^{\ell_{1} \ell_{2} \ell_{3}}+2\left(\kappa-\frac{1}{12}\right) \mathcal{A}^{\dagger}
$$

\section{D.2 Computations at first order in $\alpha^{\prime}$}

In this section we shall consider corrections at first order in $\alpha^{\prime} . \mathcal{I}_{2}$ and $\mathcal{I}_{3}$ gain $\alpha^{\prime}$ corrections from bosonic field equations and Bianchi identity, while $\mathcal{I}_{1}$ does not. Therefore we have

$$
\begin{aligned}
\mathcal{I}_{1}= & \int_{\mathcal{S}} e^{c \Phi} 2(\kappa-q)\left\langle\eta_{ \pm}, \mathcal{A}^{\dagger} \mathcal{D} \eta_{ \pm}\right\rangle+e^{c \Phi}\left(8 \kappa^{2}-2 \kappa q+q^{2}\right)\left\langle\eta_{ \pm}, \mathcal{A}^{\dagger} \mathcal{A} \eta_{ \pm}\right\rangle \\
& -\frac{1}{8} e^{c \Phi}\left\langle\eta_{ \pm}, W^{i} \ell_{1} \ell_{2} W_{i \ell_{3} \ell_{4}} \Gamma^{\ell_{1} \ell_{2} \ell_{3} \ell_{4}} \eta_{ \pm}\right\rangle+\frac{1}{16} e^{c \Phi}\left\langle\eta_{ \pm}, W_{i j k} W^{i j k} \eta_{ \pm}\right\rangle+\mathcal{O}\left(\alpha^{\prime 2}\right),
\end{aligned}
$$

and

$$
\begin{array}{rl}
\mathcal{I}_{2}=\int_{\mathcal{S}} & c e^{c \Phi}\left\langle\eta_{ \pm},\left(\Gamma^{\ell} \tilde{\nabla}_{\ell} \Phi-\frac{1}{6} W_{\ell_{1} \ell_{2} \ell_{3}} \Gamma^{\ell_{1} \ell_{2} \ell_{3}}\right) \mathcal{D} \eta_{ \pm}\right\rangle-\frac{c}{24} e^{c \Phi}\left\langle\eta_{ \pm}, W_{i j k} W^{i j k} \eta_{ \pm}\right\rangle \\
& +c\left(\frac{5}{24}-q\right) e^{c \Phi}\left\langle\eta_{ \pm}, \Gamma^{i \ell_{1} \ell_{2} \ell_{3}} \tilde{\nabla}_{i} \Phi W_{\ell_{1} \ell_{2} \ell_{3}} \eta_{ \pm}\right\rangle+e^{c \Phi}\left(\frac{1}{48}-\frac{q}{6}\right)\left\langle\eta_{ \pm}, \mathcal{A}^{\dagger} \mathcal{A} \eta_{ \pm}\right\rangle \\
& +e^{c \Phi}\left(\frac{1}{2}-2 q\right)\left\langle\eta_{ \pm}, \Gamma^{i} \tilde{\nabla}_{i} \Phi \mathcal{A} \eta_{ \pm}\right\rangle \pm e^{c \Phi}\left(\frac{1}{4}-q\right)\left\langle\eta_{ \pm}, \Gamma^{i} h_{i} \mathcal{A} \eta_{ \pm}\right\rangle \\
& +\left(\frac{3}{4}+\frac{c}{4}\right) e^{c \Phi}\left\langle\eta_{ \pm}, h_{i} h^{i} \eta_{ \pm}\right\rangle+\left(c+\frac{c^{2}}{2}+12 c q+3\right) e^{c \Phi}\left\langle\eta_{ \pm}, \tilde{\nabla}_{i} \Phi \tilde{\nabla}^{i} \Phi \eta_{ \pm}\right\rangle \\
& +\left(\frac{c}{2} \pm 6 c q \pm 3\right) e^{c \Phi}\left\langle\eta_{ \pm}, h^{i} \tilde{\nabla}_{i} \Phi \eta_{ \pm}\right\rangle \\
& -\frac{1}{24} e^{c \Phi}\left\langle\eta_{ \pm}, \Gamma^{\ell_{1} \ell_{2} \ell_{3} \ell_{4}} \tilde{\nabla}_{\ell_{1}} W_{\ell_{2} \ell_{3} \ell_{4}} \eta_{ \pm}\right\rangle+\alpha^{\prime} \frac{c}{32} e^{c \Phi}\left(-2\left\langle\eta_{ \pm}, d h_{i j} d h^{i j} \eta_{ \pm}\right\rangle\right. \\
& \left.+\left\langle\eta_{ \pm}, \tilde{\tilde{R}}_{\ell_{1} \ell_{2}, \ell_{3} \ell_{4}} \tilde{\tilde{R}}^{\ell_{1} \ell_{2}, \ell_{3} \ell_{4}} \eta_{ \pm}\right\rangle-\left\langle\eta_{ \pm}, \tilde{F}_{i j}{ }^{a b} \tilde{F}^{i j}{ }_{a b} \eta_{ \pm}\right\rangle\right)+\mathcal{O}\left(\alpha^{\prime 2}\right)
\end{array}
$$

and

$$
\begin{aligned}
\mathcal{I}_{3}=\int_{\mathcal{S}} & -\frac{5}{48} e^{c \Phi}\left\langle\eta_{ \pm}, W_{i j k} W^{i j k} \eta_{ \pm}\right\rangle+e^{c \Phi}\left\langle\eta_{ \pm}, \tilde{\nabla}_{i} \Phi \tilde{\nabla}^{i} \Phi \eta_{ \pm}\right\rangle+\frac{1}{4} e^{c \Phi}\left\langle\eta_{ \pm}, h_{i} h^{i} \eta_{ \pm}\right\rangle \\
& +e^{c \Phi}\left\langle\eta_{ \pm}, h^{i} \tilde{\nabla}_{i} \Phi \eta_{ \pm}\right\rangle+\alpha^{\prime} \frac{3}{32} e^{c \Phi}\left(-2\left\langle\eta_{ \pm}, d h_{i j} d h^{i j} \eta_{ \pm}\right\rangle\right. \\
& \left.+\left\langle\eta_{ \pm}, \check{\tilde{R}}_{\ell_{1} \ell_{2}, \ell_{3} \ell_{4}} \check{\tilde{R}}^{\ell_{1} \ell_{2}, \ell_{3} \ell_{4}} \eta_{ \pm}\right\rangle-\left\langle\eta_{ \pm}, \tilde{F}_{i j}{ }^{a b} \tilde{F}^{i j}{ }_{a b} \eta_{ \pm}\right\rangle\right)+\mathcal{O}\left(\alpha^{\prime 2}\right) .
\end{aligned}
$$


Combining all together and considering $\alpha^{\prime}$ corrections from substituting $h_{i} h^{i}$ by inverting the dilaton field equations, we have

$$
\begin{aligned}
\mathcal{I}=\int_{\mathcal{S}} e^{c \Phi}\left\langle\eta_{ \pm},\left(c \Gamma^{\ell} \tilde{\nabla}_{\ell} \Phi-\frac{1}{6} W_{\ell_{1} \ell_{2} \ell_{3}} \Gamma^{\ell_{1} \ell_{2} \ell_{3}}+2(\kappa-q) \mathcal{A}^{\dagger}\right) \mathcal{D} \eta_{ \pm}\right\rangle \\
+\left(8 \kappa^{2}-2 \kappa q-\frac{q}{12}+q^{2}\right) e^{c \Phi}\left\langle\eta_{ \pm}, \mathcal{A}^{\dagger} \mathcal{A} \eta_{ \pm}\right\rangle \\
\quad+\frac{3}{4}\left(q-\frac{1}{12}\right) e^{c \Phi}\left\langle\eta_{ \pm}, W^{i}{ }_{\ell_{1} \ell_{2}} W^{i \ell_{3} \ell_{4}} \Gamma^{\ell_{1} \ell_{2} \ell_{3} \ell_{4}} \eta_{ \pm}\right\rangle \\
\quad-c\left(q-\frac{1}{12}\right) e^{c \Phi}\left\langle\eta_{ \pm}, \Gamma^{i \ell_{1} \ell_{2} \ell_{3}} \tilde{\nabla}_{i} \Phi W_{\ell_{1} \ell_{2} \ell_{3}} \eta_{ \pm}\right\rangle+12 c\left(q+\frac{c}{24}\right) e^{c \Phi}\left\langle\eta_{ \pm}, \tilde{\nabla}_{i} \Phi \tilde{\nabla}^{i} \Phi \eta_{ \pm}\right\rangle \\
+6\left(\frac{1}{12}+q+\frac{c}{12}\right) e^{c \Phi}\left\langle\eta_{ \pm}, \tilde{\nabla}_{i} \tilde{\nabla}^{i} \Phi \eta_{ \pm}\right\rangle+\left(\frac{1}{2}-6 q \pm 6 q(c+2)\right) e^{c \Phi}\left\langle\eta_{ \pm}, h^{i} \tilde{\nabla}_{i} \Phi \eta_{ \pm}\right\rangle \\
+\frac{\alpha^{\prime}}{64} e^{c \Phi}\left(2\left\langle\eta_{ \pm}, \Gamma^{\ell_{1} \ell_{2} \ell_{3} \ell_{4}} d h_{\ell_{1} \ell_{2}} d h_{\ell_{3} \ell_{4}}\right\rangle-\left\langle\eta_{ \pm}, \Gamma^{\ell_{1} \ell_{2} \ell_{3} \ell_{4}} \check{\tilde{R}}_{\ell_{1} \ell_{2}, i j} \check{\tilde{R}}_{\left.\ell_{3} \ell_{4},{ }^{i j} \eta_{ \pm}\right\rangle}\right.\right. \\
\left.\quad+\left\langle\eta_{ \pm}, \Gamma^{\ell_{1} \ell_{2} \ell_{3} \ell_{4}} \tilde{F}_{\ell_{1} \ell_{2}, a b} \tilde{F}_{\ell_{3} \ell_{4}}{ }^{a b} \eta_{ \pm}\right\rangle\right) \\
\quad+\alpha^{\prime} \frac{3}{8}\left(\frac{1}{6}-q\right) e^{c \Phi}\left(-2\left\langle\eta_{ \pm}, d h_{i j} d h^{i j} \eta_{ \pm}\right\rangle\right. \\
\left.\quad+\left\langle\eta_{ \pm}, \tilde{\tilde{R}}_{\ell_{1} \ell_{2}, \ell_{3} \ell_{4}} \check{\tilde{R}}^{\ell_{1} \ell_{2}, \ell_{3} \ell_{4}} \eta_{ \pm}\right\rangle-\left\langle\eta_{ \pm}, \tilde{F}_{i j}{ }^{a b} \tilde{F}^{i j}{ }_{a b} \eta_{ \pm}\right\rangle\right)+\mathcal{O}\left(\alpha^{2}\right) .
\end{aligned}
$$

To further simplify (D.24), we note the following identity

$$
\begin{aligned}
\left\langle\eta_{ \pm}, \Gamma^{\ell_{1} \ell_{2} \ell_{3} \ell_{4}} d h_{\ell_{1} \ell_{2}} d h_{\ell_{3} \ell_{4}} \eta_{ \pm}\right\rangle= & \left\langle\eta_{ \pm}, \Gamma^{\ell_{1} \ell_{2}} d h_{\ell_{1} \ell_{2}} \Gamma^{\ell_{3} \ell_{4}} d h_{\ell_{3} \ell_{4}} \eta_{ \pm}\right\rangle \\
& +2\left\langle\eta_{ \pm}, d h_{i j} d h^{i j} \eta_{ \pm}\right\rangle
\end{aligned}
$$

Identities analogous to (D.25) hold also for the terms which involve $\check{\tilde{R}}_{i j, k \ell}$ and $\tilde{F}_{i j}{ }^{a b}$. This leads to

$$
\begin{aligned}
\mathcal{I}=\int_{\mathcal{S}} & e^{c \Phi}\left\langle\eta_{ \pm},\left(c \Gamma^{\ell} \tilde{\nabla}_{\ell} \Phi-\frac{1}{6} W_{\ell_{1} \ell_{2} \ell_{3}} \Gamma^{\ell_{1} \ell_{2} \ell_{3}}+2(\kappa-q) \mathcal{A}^{\dagger}\right) \mathcal{D} \eta_{ \pm}\right\rangle \\
& +\left(8 \kappa^{2}-2 \kappa q-\frac{q}{12}+q^{2}\right) e^{c \Phi}\left\langle\eta_{ \pm}, \mathcal{A}^{\dagger} \mathcal{A} \eta_{ \pm}\right\rangle \\
& +\frac{3}{4}\left(q-\frac{1}{12}\right) e^{c \Phi}\left\langle\eta_{ \pm}, W^{i} \ell_{1} \ell_{2} W^{i \ell_{3} \ell_{4}} \Gamma^{\ell_{1} \ell_{2} \ell_{3} \ell_{4}} \eta_{ \pm}\right\rangle \\
& -c\left(q-\frac{1}{12}\right) e^{c \Phi}\left\langle\eta_{ \pm}, \Gamma^{i \ell_{1} \ell_{2} \ell_{3}} \tilde{\nabla}_{i} \Phi W_{\ell_{1} \ell_{2} \ell_{3}} \eta_{ \pm}\right\rangle+12 c\left(q+\frac{c}{24}\right) e^{c \Phi}\left\langle\eta_{ \pm}, \tilde{\nabla}_{i} \Phi \tilde{\nabla}^{i} \Phi \eta_{ \pm}\right\rangle \\
& +6\left(\frac{1}{12}+q+\frac{c}{12}\right) e^{c \Phi}\left\langle\eta_{ \pm}, \tilde{\nabla}_{i} \tilde{\nabla}^{i} \Phi \eta_{ \pm}\right\rangle+\left(\frac{1}{2}-6 q \pm 6 q(c+2)\right) e^{c \Phi}\left\langle\eta_{ \pm}, h^{i} \tilde{\nabla}_{i} \Phi \eta_{ \pm}\right\rangle \\
& +\frac{3}{8} \alpha^{\prime}\left(q-\frac{1}{12}\right) e^{c \Phi}\left(2 d h_{i j} d h^{i j}+\tilde{F}_{i j}{ }^{a b} \tilde{F}^{i j}{ }_{a b}-\check{\tilde{R}}_{\ell_{1} \ell_{2}, \ell_{3} \ell_{4}} \check{\tilde{R}}^{\ell_{1} \ell_{2}, \ell_{3} \ell_{4}}\right)\left\|\eta_{ \pm}\right\|^{2} \\
& -\frac{\alpha^{\prime}}{32} e^{c \Phi}\left\|d / h \eta_{ \pm}\right\|^{2}-\frac{\alpha^{\prime}}{64} e^{c \Phi}\left\|\tilde{F}^{*} \eta_{ \pm}\right\|^{2}+\frac{\alpha^{\prime}}{64} e^{c \Phi}\left\langle\check{\tilde{R}}_{\ell_{1} \ell_{2}, i j} \Gamma^{\ell_{1} \ell_{2}} \eta_{ \pm}, \check{\tilde{R}}_{\ell_{3} \ell_{4},}^{i j} \Gamma^{\ell_{3} \ell_{4}} \eta_{ \pm}\right\rangle \\
& +\mathcal{O}\left(\alpha^{\prime 2}\right) .
\end{aligned}
$$


In order to eliminate the term $\left\langle\eta_{ \pm}, W^{i} \ell_{1} \ell_{2} W_{i \ell_{3} \ell_{4}} \Gamma^{\ell_{1} \ell_{2} \ell_{3} \ell_{4}} \eta_{ \pm}\right\rangle$, which has no sign and cannot be rewritten in terms of $\mathcal{D}$ or $\mathcal{A}^{\dagger} \mathcal{A}$, we must set

$$
q=\frac{1}{12}+\mathcal{O}\left(\alpha^{\prime 2}\right)
$$

and then in order to eliminate the $\left\langle\eta_{ \pm}, \tilde{\nabla}^{i} \tilde{\nabla}_{i} \Phi \eta_{ \pm}\right\rangle$term we must further set

$$
c=-2+\mathcal{O}\left(\alpha^{\prime 2}\right) .
$$

Then (D.26) is significantly simplified to

$$
\begin{aligned}
\mathcal{I}= & \left(8 \kappa^{2}-\frac{1}{6} \kappa\right) \int_{\mathcal{S}} e^{-2 \Phi}\left\|\mathcal{A} \eta_{ \pm}\right\|^{2}+\int_{\mathcal{S}} e^{-2 \Phi}\left\langle\eta_{ \pm}, \Psi \mathcal{D} \eta_{ \pm}\right\rangle \\
& -\frac{\alpha^{\prime}}{64} \int_{\mathcal{S}} e^{-2 \Phi}\left(2\left\|d / h \eta_{ \pm}\right\|^{2}+\left\|\mathscr{F}_{ \pm}\right\|^{2}-\left\langle\check{\tilde{R}}_{\ell_{1} \ell_{2}, i j} \Gamma^{\ell_{1} \ell_{2}} \eta_{ \pm}, \check{\tilde{R}}_{\ell_{3} \ell_{4}}^{i j}, \Gamma^{\ell_{3} \ell_{4}} \eta_{ \pm}\right\rangle\right)+\mathcal{O}\left(\alpha^{\prime 2}\right),
\end{aligned}
$$

where $\Psi$ is defined in (D.20).

\section{E $\quad \operatorname{AdS}_{n+1}$ as warped product over $\mathrm{AdS}_{n}$}

The $\mathrm{AdS}_{n+1}$ space can be written as a warped product over $\mathrm{AdS}_{n}$. This has been observed before in [45] for $\mathrm{AdS}_{3}$ and elsewhere, see eg [47]. For this, we label all geometrical objects defined on $\operatorname{AdS}_{n+1}$ and $\operatorname{AdS}_{n}$ by $n+1$ and $n$ respectively, e.g. $d s_{n+1}^{2}$ is the metric on $\operatorname{AdS}_{n+1}$ and $d s_{n}^{2}$ is the metric on $\mathrm{AdS}_{n}$. In principle $\mathrm{AdS}_{n+1}$ and $\mathrm{AdS}_{n}$ can have different radii, which are indicated by $\ell_{n+1}$ and $\ell_{n}$ respectively. Coordinates on $\operatorname{AdS}_{n+1}$ are taken to be as follows

$$
x^{I}=\left(x^{0}, x^{i}\right), \quad x^{0} \equiv y, \quad i=1, \ldots, n .
$$

We shall begin with an Ansätz for the metric on $\mathrm{AdS}_{n+1}$ as a warped product over $\operatorname{AdS}_{n}$, i.e.

$$
d s_{n+1}^{2}=d y^{2}+f(y)^{2} d s_{n}^{2} .
$$

We want to determine the necessary and sufficient conditions to impose on $f(y)$ in order for $d s_{n+1}^{2}$ to be the metric on $\operatorname{AdS}_{n+1}$. To succeed, we have to impose the fact $\operatorname{AdS}_{n+1}$ is a maximally symmetric space. Locally, the necessary and sufficient condition is that the Riemann tensor must assume the following form

$$
R_{I J K L}^{(n+1)}=-\frac{1}{\ell_{n+1}^{2}}\left(g_{I K}^{(n+1)} g_{J L}^{(n+1)}-g_{J K}^{(n+1)} g_{I L}^{(n+1)}\right)
$$

Equation (E.3) implies also that the metric (E.2) is Einstein and the curvature scalar is constant and negative, i.e.

$$
R_{I J}^{(n+1)}=-\frac{n}{\ell_{n+1}^{2}} g_{I J}^{(n+1)}, \quad \quad R^{(n+1)}=-\frac{1}{\ell_{n+1}^{2}} n(n+1) .
$$


The non-vanishing Christoffel symbols of (E.2) are:

$$
\Gamma_{i 0}^{(n+1) k}=\frac{f^{\prime}(y)}{f(y)} \delta_{i}^{k}, \quad \Gamma_{i j}^{(n+1) 0}=-f(y) f^{\prime}(y) g_{i j}^{(n+1)}, \quad \Gamma_{i j}^{(n+1) k}=\Gamma_{i j}^{(n) k} .
$$

The non-vanishing Riemann tensor components are:

$$
\begin{aligned}
& R^{(n+1)}{ }_{i 0,}{ }^{k}{ }_{0}=-\frac{f^{\prime \prime}(y)}{f(y)} \delta^{k}{ }_{i}, \\
& R^{(n+1)}{ }_{i 0}{ }^{0}{ }_{\ell}=f(y) f^{\prime \prime}(y) g_{i \ell}^{(n)}, \\
& R^{(n+1)}{ }_{i j,{ }^{k}{ }_{\ell}}=R^{(n)}{ }_{i j}{ }^{k}{ }{ }_{\ell}+f^{\prime}(y)^{2}\left(\delta^{k}{ }_{j} g_{i \ell}^{(n)}-\delta^{k}{ }_{i} g_{j \ell}^{(n)}\right),
\end{aligned}
$$

and

$$
\begin{aligned}
& R_{i 0, k 0}^{(n+1)}=-f(y) f^{\prime \prime}(y) g_{i k}^{(n)} \\
& R_{i j, k l}^{(n+1)}=f(y)^{2} R_{i j, k l}^{(n)}-f(y)^{2} f^{\prime}(y)^{2}\left(g_{i k}^{(n)} g_{j l}^{(n)}-g_{j k}^{(n)} g_{i l}^{(n)}\right) .
\end{aligned}
$$

The non-vanishing Ricci tensor components are:

$$
\begin{aligned}
& R_{00}^{(n+1)}=-n \frac{f^{\prime \prime}(y)}{f(y)}, \\
& R_{i j}^{(n+1)}=R_{i j}^{(n)}+\left[f^{\prime}(y)^{2}(1-n)-f(y) f^{\prime \prime}(y)\right] g_{i j}^{(n)} .
\end{aligned}
$$

The Riemann tensor on $\mathrm{AdS}_{n}$ must assume the following form

$$
R_{i j k \ell}^{(n)}=-\frac{1}{\ell_{n}^{2}}\left(g_{i k}^{(n)} g_{j \ell}^{(n)}-g_{j k}^{(n)} g_{i \ell}^{(n)}\right) .
$$

Now we impose (E.3). The $(i 0, k 0)$-components provide the first ordinary differential equation for $f$

$$
f^{\prime \prime}(y)=\frac{1}{\ell_{n+1}^{2}} f(y) .
$$

The $(i j, k l)$-components provide the second ordinary differential equation for $f$

$$
f^{\prime}(y)^{2}-\frac{1}{\ell_{n+1}^{2}} f(y)^{2}+\frac{1}{\ell_{n}^{2}}=0 .
$$

Since equations in (E.4) are derived from (E.3), they would imply again (E.10) and (E.11), so there is nothing further to be learned from those conditions. The general solution of (E.10) and (E.11) is

$$
f(y)=\alpha \cosh \left(\frac{y}{\ell_{n+1}}\right)+\beta \sinh \left(\frac{y}{\ell_{n+1}}\right),
$$

where $\alpha$ and $\beta$ are constants which satisfy

$$
\alpha^{2}-\beta^{2}=\frac{\ell_{n+1}^{2}}{\ell_{n}^{2}} .
$$


The solution (E.12) leads us to the following conclusions

1. if $y \in(-\infty,+\infty)$, then locally the $\mathrm{AdS}_{3}$ metric can be written as $\operatorname{AdS}_{2} \times_{w} \mathbb{R}$.

2. if $y \in[0,1]$, then locally the $\mathrm{AdS}_{3}$ metric can be written as $\mathrm{AdS}_{2} \times_{w}[0,1]$ as the warp factor is not periodic.

3. if $y \in[0,1]$ and force periodicity on $y$, then the metric of $\operatorname{AdS}_{2} \times_{w} S^{1}$ is discontinuous as the warp factor is not periodic.

From the perspective of near horizon geometries, the first case violates the compactness condition of the partial horizon section. The second case implies that the spatial horizon section has a boundary. The third case violates smoothness condition since (E.12) is not periodic. Hence all cases violate one or more of the assumptions required to prove that there are no $\mathrm{AdS}_{2}$ horizons in the heterotic theory.

Open Access. This article is distributed under the terms of the Creative Commons Attribution License (CC-BY 4.0), which permits any use, distribution and reproduction in any medium, provided the original author(s) and source are credited.

\section{References}

[1] W. Israel, Event horizons in static vacuum space-times, Phys. Rev. 164 (1967) 1776 [INSPIRE].

[2] B. Carter, Axisymmetric Black Hole Has Only Two Degrees of Freedom, Phys. Rev. Lett. 26 (1971) 331 [INSPIRE].

[3] S.W. Hawking, Black holes in general relativity, Commun. Math. Phys. 25 (1972) 152 [INSPIRE].

[4] D.C. Robinson, Uniqueness of the Kerr black hole, Phys. Rev. Lett. 34 (1975) 905 [INSPIRE].

[5] W. Israel, Event horizons in static electrovac space-times, Commun. Math. Phys. 8 (1968) 245 [INSPIRE].

[6] P.O. Mazur, Proof of uniqueness of the Kerr-Newman black hole solution, J. Phys. A 15 (1982) 3173 [inSPIRE].

[7] D. Robinson, Four decades of black hole uniqueness theorems, in The Kerr spacetime: Rotating black holes in General Relativity, D.L. Wiltshire, M. Visser and S.M. Scott eds., Cambridge University Press (2009), pg. 115-143.

[8] R. Emparan and H.S. Reall, A rotating black ring solution in five-dimensions, Phys. Rev. Lett. 88 (2002) 101101 [hep-th/0110260] [INSPIRE].

[9] J. Gutowski and G. Papadopoulos, Index theory and dynamical symmetry enhancement of M-horizons, JHEP 05 (2013) 088 [arXiv: 1303.0869] [INSPIRE].

[10] U. Gran, J. Gutowski and G. Papadopoulos, Index theory and dynamical symmetry enhancement near IIB horizons, JHEP 11 (2013) 104 [arXiv:1306.5765] [INSPIRE].

[11] U. Gran, J. Gutowski, U. Kayani and G. Papadopoulos, Dynamical symmetry enhancement near IIA horizons, JHEP 06 (2015) 139 [arXiv: 1409.6303] [INSPIRE]. 
[12] U. Gran, J. Gutowski, U. Kayani and G. Papadopoulos, Dynamical symmetry enhancement near massive IIA horizons, Class. Quant. Grav. 32 (2015) 235004 [arXiv:1411.5286] [INSPIRE].

[13] M.F. Atiyah and I.M. Singer, The index of elliptic operators. 1, Annals Math. 87 (1968) 484 [INSPIRE].

[14] R. Kallosh and A.W. Peet, Dilaton black holes near the horizon, Phys. Rev. D 46 (1992) R5223 [hep-th/9209116] [InSPIRE].

[15] S. Ferrara and R. Kallosh, Supersymmetry and attractors, Phys. Rev. D 54 (1996) 1514 [hep-th/9602136] [INSPIRE].

[16] G.W. Gibbons and P.K. Townsend, Vacuum interpolation in supergravity via super p-branes, Phys. Rev. Lett. 71 (1993) 3754 [hep-th/9307049] [INSPIRE].

[17] J.C. Breckenridge, R.C. Myers, A.W. Peet and C. Vafa, D-branes and spinning black holes, Phys. Lett. B 391 (1997) 93 [hep-th/9602065] [inSPIRE].

[18] A.H. Chamseddine, S. Ferrara, G.W. Gibbons and R. Kallosh, Enhancement of supersymmetry near 5-D black hole horizon, Phys. Rev. D 55 (1997) 3647 [hep-th/9610155] [INSPIRE].

[19] J.B. Gutowski and H.S. Reall, Supersymmetric AdS $S_{5}$ black holes, JHEP 02 (2004) 006 [hep-th/0401042] [INSPIRE].

[20] K. Hanaki, K. Ohashi and Y. Tachikawa, Supersymmetric Completion of an $R^{2}$ term in Five-dimensional Supergravity, Prog. Theor. Phys. 117 (2007) 533 [hep-th/0611329] [INSPIRE].

[21] J.B. Gutowski, D. Klemm, W.A. Sabra and P. Sloane, Small Horizons, JHEP 01 (2012) 146 [arXiv: 1109.1566] [INSPIRE].

[22] J.B. Gutowski, Uniqueness of five-dimensional supersymmetric black holes, JHEP 08 (2004) 049 [hep-th/0404079] [INSPIRE].

[23] A. Castro, J.L. Davis, P. Kraus and F. Larsen, String Theory Effects on Five-Dimensional Black Hole Physics, Int. J. Mod. Phys. A 23 (2008) 613 [arXiv:0801.1863] [InSPIRE].

[24] N.S. Manton, Vortex solutions of the Popov equations, J. Phys. A 46 (2013) 145402 [arXiv: 1211.4352] [INSPIRE].

[25] U. Gran, P. Lohrmann and G. Papadopoulos, The spinorial geometry of supersymmetric heterotic string backgrounds, JHEP 02 (2006) 063 [hep-th/0510176] [INSPIRE].

[26] U. Gran, G. Papadopoulos, D. Roest and P. Sloane, Geometry of all supersymmetric type-I backgrounds, JHEP 08 (2007) 074 [hep-th/0703143] [INSPIRE].

[27] J. Gutowski and G. Papadopoulos, Heterotic Black Horizons, JHEP 07 (2010) 011 [arXiv: 0912.3472] [INSPIRE].

[28] G. Papadopoulos, Heterotic supersymmetric backgrounds with compact holonomy revisited, Class. Quant. Grav. 27 (2010) 125008 [arXiv:0909.2870] [INSPIRE].

[29] J. Gillard, G. Papadopoulos and D. Tsimpis, Anomaly, fluxes and (2,0) heterotic string compactifications, JHEP 06 (2003) 035 [hep-th/0304126] [INSPIRE].

[30] C.G. Callan Jr., J.A. Harvey and A. Strominger, Supersymmetric string solitons, hep-th/9112030 [INSPIRE]. 
[31] P.S. Howe and G. Papadopoulos, Finiteness and anomalies in $(4,0)$ supersymmetric $\sigma$-models, Nucl. Phys. B 381 (1992) 360 [hep-th/9203070] [INSPIRE].

[32] M.B. Green, J.H. Schwarz and E. Witten, Superstring Theory, vols. 1 and 2, Cambridge Monographs On Mathematical Physics, Cambridge University Press (1987).

[33] S.W. Beck, J.B. Gutowski and G. Papadopoulos, Geometry and supersymmetry of heterotic warped flux AdS backgrounds, JHEP 07 (2015) 152 [arXiv:1505.01693] [INSPIRE].

[34] V. Moncrief and J. Isenberg, Symmetries of cosmological Cauchy horizons, Commun. Math. Phys. 89 (1983) 387 [INSPIRE].

[35] H. Friedrich, I. Racz and R.M. Wald, On the rigidity theorem for space-times with a stationary event horizon or a compact Cauchy horizon, Commun. Math. Phys. 204 (1999) 691 [gr-qc/9811021] [InSPIRE].

[36] M. Protter and H. Weinberger, Maximum principles in differential equations, Prentice-Hall Inc. (1967).

[37] J.B. Gutowski and G. Papadopoulos, Supersymmetry of AdS and flat backgrounds in M-theory, JHEP 02 (2015) 145 [arXiv: 1407.5652] [INSPIRE].

[38] S.W. Beck, J.B. Gutowski and G. Papadopoulos, Supersymmetry of AdS and flat IIB backgrounds, JHEP 02 (2015) 020 [arXiv:1410.3431] [INSPIRE].

[39] S. Beck, J.B. Gutowski and G. Papadopoulos, Supersymmetry of IIA warped flux AdS and flat backgrounds, JHEP 09 (2015) 135 [arXiv:1501.07620] [INSPIRE].

[40] S. Ivanov, Connection with torsion, parallel spinors and geometry of $\operatorname{Spin}(7)$ manifolds, math/0111216 [INSPIRE].

[41] T. Friedrich and S. Ivanov, Killing spinor equations in dimension 7 and geometry of integrable $G_{2}$ manifolds, J. Geom. Phys. 48 (2003) 1 [math/0112201] [INSPIRE].

[42] P.S. Howe, G. Papadopoulos and V. Stojevic, Covariantly constant forms on torsionful geometries from world-sheet and spacetime perspectives, JHEP 09 (2010) 100 [arXiv: 1004.2824] [INSPIRE].

[43] P.S. Howe and G. Papadopoulos, Twistor spaces for HKT manifolds, Phys. Lett. B 379 (1996) 80 [hep-th/9602108] [INSPIRE].

[44] P.S. Howe, A. Opfermann and G. Papadopoulos, Twistor spaces for QKT manifolds, Commun. Math. Phys. 197 (1998) 713 [hep-th/9710072] [INSPIRE].

[45] A. Strominger, AdS 2 quantum gravity and string theory, JHEP 01 (1999) 007 [hep-th/9809027] [INSPIRE].

[46] J. Gillard, G. Papadopoulos and D. Tsimpis, Anomaly, fluxes and (2,0) heterotic string compactifications, JHEP 06 (2003) 035 [hep-th/0304126] [INSPIRE].

[47] A.B. Clark, N. Crossette, G.M. Newman and A. Rommal, AdS-Sliced Flavor Branes and Adding Flavor to the Janus Solution, Phys. Rev. D 89 (2014) 026014 [arXiv:1309.7872] [INSPIRE]. 